\title{
Understanding the Impact of Stimulation Treatment on Gas Production from a Horizontally Drilled Marcellus Shale Well
}

\author{
Matthew Dean Carl Perrella
}

Follow this and additional works at: https://researchrepository.wvu.edu/etd

\section{Recommended Citation}

Perrella, Matthew Dean Carl, "Understanding the Impact of Stimulation Treatment on Gas Production from a Horizontally Drilled Marcellus Shale Well" (2018). Graduate Theses, Dissertations, and Problem Reports. 6405.

https://researchrepository.wvu.edu/etd/6405

This Thesis is protected by copyright and/or related rights. It has been brought to you by the The Research Repository @ WVU with permission from the rights-holder(s). You are free to use this Thesis in any way that is permitted by the copyright and related rights legislation that applies to your use. For other uses you must obtain permission from the rights-holder(s) directly, unless additional rights are indicated by a Creative Commons license in the record and/ or on the work itself. This Thesis has been accepted for inclusion in WVU Graduate Theses, Dissertations, and Problem Reports collection by an authorized administrator of The Research Repository @ WVU. For more information, please contact researchrepository@mail.wvu.edu. 
Understanding the Impact of Stimulation Treatment on Gas Production from a Horizontally Drilled Marcellus Shale Well

\author{
Matthew Dean Carl Perrella
}

Thesis submitted

to the Petroleum and Natural Gas Engineering Department at West Virginia University

in partial fulfillment of the requirements for the degree of

Master of Science in

Petroleum and Natural Gas Engineering

Sam Ameri, Ph.D, Chair

Ilkin Bilgesu, Ph.D.

Kashy Aminian, Ph.D.

Department of Petroleum and Natural Gas Engineering

Morgantown, West Virginia

2018

Keywords: [flow scanner, neural network, gas, completion design]

Copyright 2018 Matthew Dean Carl Perrella 


\section{ABSTRACT \\ Understanding the Impact of Stimulation Treatment on Gas Production from a Horizontally Drilled Marcellus Shale Well}

\section{Matthew Dean Carl Perrella}

The Marcellus shale is one of the largest unconventional gas shale plays in the United States. It underlies much of Pennsylvania, West Virginia, and New York and even extends under Lake Erie and into Canada. The most effective methods to produce from this play is to drill horizontally into the shale formation and use hydraulic fracturing. Hydraulic fracturing creates pathways for the hydrocarbon to flow from the shale into the wellbore. These lateral sections of the well are typically completed from toe to heel over a number of stages using a plug and perforate method.

This research focuses on a Marcellus shale well drilled in Morgantown, West Virginia. The well was completed using five different fracture designs over a total of 28 stages throughout the lateral. This well served as more of a learning experience than a typical horizontal well. A flow scanner was also run through the well after hydraulic fracturing to discover more information that is typically not acquired in most wells. All data for this well was provided by the Marcellus Shale Energy and Environment Laboratory (MSEEL) research group. The MSEEL participants were Northeastern Natural Energy, Department of Energy, West Virginia University, Ohio State University, and others who reviewed all the collected information. The goal of research group was to improve the understanding the shale characteristics in this region in order to be more efficient in the completion of other wells in this location.

A neural network model was used to examine the efficiency and performance of different completion methods and their impact on gas production. Several input parameters such as plug depth, total shots, natural fractures, measured slurry, measured clean fluid, measured proppant, pump time and stage length were used to predict gas flowrate. Several combinations of training, validation, and testing sets were employed with different number of hidden layer neurons and the best combination was determined. 


\section{Table of Contents}

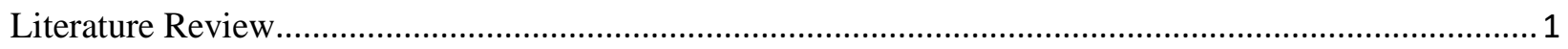

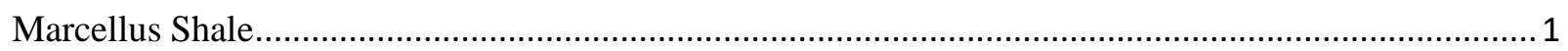

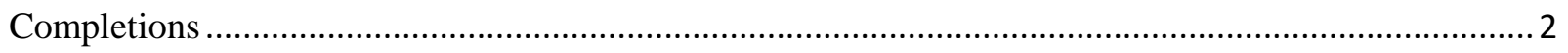

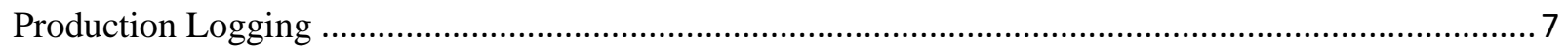

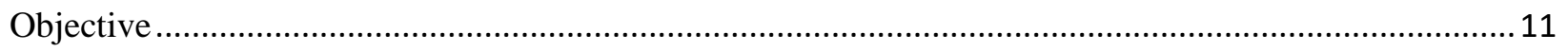

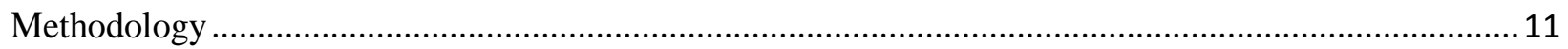

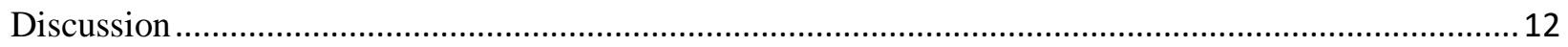

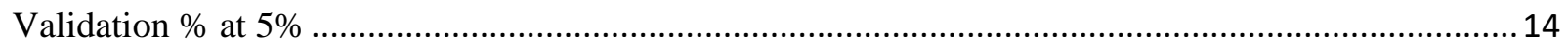

90\% Training, 5\% Validation, 5\% Testing and 10 Hidden Neurons .................................................. 15

85\% Training, 5\% Validation, 10\% Testing and 10 Hidden Neurons ............................................... 16

80\% Training, 5\% Validation, 15\% Testing and 10 Hidden Neurons ............................................. 17

75\% Training, 5\% Validation, 20\% Testing and 10 Hidden Neurons .............................................. 18

70\% Training, 5\% Validation, 25\% Testing and 10 Hidden Neurons ............................................. 19

65\% Training, 5\% Validation, 30\% Testing and 10 Hidden Neurons ................................................20

60\% Training, 5\% Validation, 35\% Testing and 10 Hidden Neurons ............................................... 21

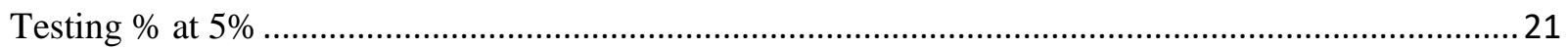


$85 \%$ Training, $10 \%$ Validation, 5\% Testing and 10 Hidden Neurons ...

$80 \%$ Training, $10 \%$ Validation, $10 \%$ Testing and 10 Hidden Neurons 22

75\% Training, $10 \%$ Validation, $15 \%$ Testing and 10 Hidden Neurons 23

70\% Training, 10\% Validation, 20\% Testing and 10 Hidden Neurons 24

$65 \%$ Training, $10 \%$ Validation, $25 \%$ Testing and 10 Hidden Neurons .25

$60 \%$ Training, $10 \%$ Validation, 30\% Testing and 10 Hidden Neurons .26

55\% Training, 10\% Validation, 35\% Testing and 10 Hidden Neurons .27

$80 \%$ Training, $15 \%$ Validation, 5\% Testing and 10 Hidden Neurons .28

75\% Training, $15 \%$ Validation, $10 \%$ Testing and 10 Hidden Neurons 29

70\% Training, $15 \%$ Validation, $15 \%$ Testing and 10 Hidden Neurons 30

$65 \%$ Training, 15\% Validation, 20\% Testing and 10 Hidden Neurons 31

$60 \%$ Training, $15 \%$ Validation, 25\% Testing and 10 Hidden Neurons 32

55\% Training, $15 \%$ Validation, $30 \%$ Testing and 10 Hidden Neurons 33

50\% Training, 15\% Validation, 35\% Testing and 10 Hidden Neurons 34

75\% Training, 20\% Validation, 5\% Testing and 10 Hidden Neurons 35

70\% Training, 20\% Validation, 10\% Testing and 10 Hidden Neurons 36

65\% Training, 20\% Validation, 15\% Testing and 10 Hidden Neurons 37

$60 \%$ Training, 20\% Validation, 20\% Testing and 10 Hidden Neurons .38

55\% Training, 20\% Validation, 25\% Testing and 10 Hidden Neurons 39

50\% Training, 20\% Validation, 30\% Testing and 10 Hidden Neurons 40 
45\% Training, 20\% Validation, 35\% Testing and 10 Hidden Neurons

70\% Training, 25\% Validation, 5\% Testing and 10 Hidden Neurons 42

65\% Training, 25\% Validation, 10\% Testing and 10 Hidden Neurons 43

$60 \%$ Training, 25\% Validation, $15 \%$ Testing and 10 Hidden Neurons 44

55\% Training, 25\% Validation, 20\% Testing and 10 Hidden Neurons 45

50\% Training, 25\% Validation, 25\% Testing and 10 Hidden Neurons .46

45\% Training, 25\% Validation, 30\% Testing and 10 Hidden Neurons .47

40\% Training, 25\% Validation, 35\% Testing and 10 Hidden Neurons .48

65\% Training, 30\% Validation, 5\% Testing and 10 Hidden Neurons 49

$60 \%$ Training, 30\% Validation, $10 \%$ Testing and 10 Hidden Neurons 50 55\% Training, 30\% Validation, 15\% Testing and 10 Hidden Neurons 51

50\% Training, 30\% Validation, 20\% Testing and 10 Hidden Neurons 52

45\% Training, 30\% Validation, 25\% Testing and 10 Hidden Neurons .53

40\% Training, 30\% Validation, 30\% Testing and 10 Hidden Neurons .54

35\% Training, 30\% Validation, 35\% Testing and 10 Hidden Neurons .55

$60 \%$ Training, 35\% Validation, $5 \%$ Testing and 10 Hidden Neurons .56

55\% Training, 35\% Validation, 10\% Testing and 10 Hidden Neurons .57

50\% Training, 35\% Validation, 15\% Testing and 10 Hidden Neurons .58

45\% Training, 35\% Validation, 20\% Testing and 10 Hidden Neurons 59

40\% Training, 35\% Validation, 25\% Testing and 10 Hidden Neurons 60 
35\% Training, 35\% Validation, 30\% Testing and 10 Hidden Neurons

$30 \%$ Training, 35\% Validation, 35\% Testing and 10 Hidden Neurons 62

Varying Number of Hidden Neurons in Best Runs 63

75\% Training, 10\% Validation, 15\% Testing and 5 Hidden Neurons ............................................64

75\% Training, 10\% Validation, 15\% Testing and 15 Hidden Neurons .......................................... 64

75\% Training, 15\% Validation, 10\% Testing and 5 Hidden Neurons .......................................... 65

75\% Training, 15\% Validation, 10\% Testing and 15 Hidden Neurons ..........................................66

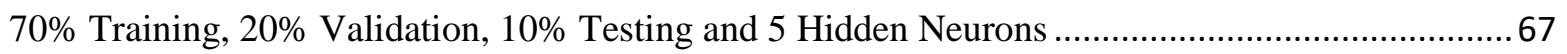

70\% Training, 20\% Validation, 10\% Testing and 15 Hidden Neurons .......................................... 68

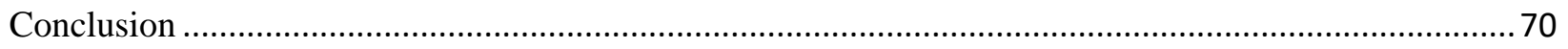

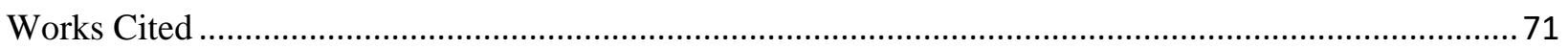


Figure 1: 90\% Training, 5\% Validation, 5\% Testing and 10 Hidden Neurons 15

Figure 2: 85\% Training, 5\% Validation, 10\% Testing and 10 Hidden Neurons...................................... 16

Figure 3: 80\% Training, 5\% Validation, 15\% Testing and 10 Hidden Neurons..................................... 17

Figure 4: 75\% Training, 5\% Validation, 20\% Testing and 10 Hidden Neurons..................................... 18

Figure 5: 70\% Training, 5\% Validation, 25\% Testing and 10 Hidden Neurons...................................... 19

Figure 6: 65\% Training, 5\% Validation, 30\% Testing and 10 Hidden Neurons...................................... 20

Figure 7: 60\% Training, 5\% Validation, 35\% Testing and 10 Hidden Neurons..................................... 21

Figure 8: 85\% Training, 10\% Validation, 5\% Testing and 10 Hidden Neurons......................................22

Figure 9: $80 \%$ Training, 10\% Validation, 10\% Testing and 10 Hidden Neurons.....................................2 23

Figure 10: 75\% Training, 10\% Validation, 15\% Testing and 10 Hidden Neurons.................................. 24

Figure 11: 70\% Training, 10\% Validation, 20\% Testing and 10 Hidden Neurons...................................25

Figure 12: 65\% Training, 10\% Validation, 25\% Testing and 10 Hidden Neurons...................................26

Figure 13: 60\% Training, 10\% Validation, 30\% Testing and 10 Hidden Neurons.................................. 27

Figure 14: 55\% Training, 10\% Validation, 35\% Testing and 10 Hidden Neurons...................................28

Figure 15: 80\% Training, 15\% Validation, 5\% Testing and 10 Hidden Neurons....................................29

Figure 16: 75\% Training, 15\% Validation, 10\% Testing and 10 Hidden Neurons................................... 30

Figure 17: 70\% Training, 15\% Validation, 15\% Testing and 10 Hidden Neurons................................... 31

Figure 18: 65\% Training, 15\% Validation, 20\% Testing and 10 Hidden Neurons.................................. 32

Figure 19: 60\% Training, 15\% Validation, 25\% Testing and 10 Hidden Neurons.................................. 33

Figure 20: 55\% Training, 15\% Validation, 30\% Testing and 10 Hidden Neurons................................... 34

Figure 21: 50\% Training, 15\% Validation, 35\% Testing and 10 Hidden Neurons................................... 35

Figure 22: 75\% Training, 20\% Validation, 5\% Testing and 10 Hidden Neurons..................................... 36

Figure 23: 70\% Training, 20\% Validation, 10\% Testing and 10 Hidden Neurons................................... 37 
Figure 24: 65\% Training, 20\% Validation, 15\% Testing and 10 Hidden Neurons

Figure 25: 60\% Training, 20\% Validation, 20\% Testing and 10 Hidden Neurons....................................39

Figure 26: 55\% Training, 20\% Validation, 25\% Testing and 10 Hidden Neurons.................................. 40

Figure 27: 50\% Training, 20\% Validation, 30\% Testing and 10 Hidden Neurons.................................. 41

Figure 28: 45\% Training, 20\% Validation, 35\% Testing and 10 Hidden Neurons.................................. 42

Figure 29: 70\% Training, 25\% Validation, 5\% Testing and 10 Hidden Neurons.................................... 43

Figure 30: 65\% Training, 25\% Validation, 10\% Testing and 10 Hidden Neurons................................. 44

Figure 31: 60\% Training, 25\% Validation, 15\% Testing and 10 Hidden Neurons................................... 45

Figure 32: 55\% Training, 25\% Validation, 20\% Testing and 10 Hidden Neurons.................................. 46

Figure 33: 50\% Training, 25\% Validation, 25\% Testing and 10 Hidden Neurons.................................47

Figure 34: 45\% Training, 25\% Validation, 30\% Testing and 10 Hidden Neurons.................................... 48

Figure 35: 40\% Training, 25\% Validation, 35\% Testing and 10 Hidden Neurons.................................. 49

Figure 36: 65\% Training, 30\% Validation, 5\% Testing and 10 Hidden Neurons....................................50

Figure 37: 60\% Training, 30\% Validation, 10\% Testing and 10 Hidden Neurons................................... 51

Figure 38: 55\% Training, 30\% Validation, 15\% Testing and 10 Hidden Neurons..................................52

Figure 39: 50\% Training, 30\% Validation, 20\% Testing and 10 Hidden Neurons................................. 53

Figure 40: 45\% Training, 30\% Validation, 25\% Testing and 10 Hidden Neurons..................................54

Figure 41: 40\% Training, 30\% Validation, 30\% Testing and 10 Hidden Neurons..................................55

Figure 42: 35\% Training, 30\% Validation, 35\% Testing and 10 Hidden Neurons..................................56

Figure 43: 60\% Training, 35\% Validation, 5\% Testing and 10 Hidden Neurons....................................57

Figure 44: 55\% Training, 35\% Validation, 10\% Testing and 10 Hidden Neurons..................................58

Figure 45: 50\% Training, 35\% Validation, 15\% Testing and 10 Hidden Neurons..................................59

Figure 46: 45\% Training, 35\% Validation, 20\% Testing and 10 Hidden Neurons.................................. 60

Figure 47: 40\% Training, 35\% Validation, 25\% Testing and 10 Hidden Neurons.................................. 61 
Figure 48: 35\% Training, 35\% Validation, 30\% Testing and 10 Hidden Neurons..................................62

Figure 49: 30\% Training, 35\% Validation, 35\% Testing and 10 Hidden Neurons.................................63

Figure 50: 75\% Training, 10\% Validation, 15\% Testing and 5 Hidden Neurons.................................... 64

Figure 51: 75\% Training, 10\% Validation, 15\% Testing and 15 Hidden Neurons................................. 65

Figure 52: 75\% Training, 15\% Validation, 10\% Testing and 5 Hidden Neurons...................................66

Figure 53: 75\% Training, 15\% Validation, 10\% Testing and 15 Hidden Neurons.................................. 67

Figure 54: 70\% Training, 20\% Validation, 10\% Testing and 5 Hidden Neurons....................................68

Figure 55: 70\% Training, 20\% Validation, 10\% Testing and 15 Hidden Neurons................................... 69

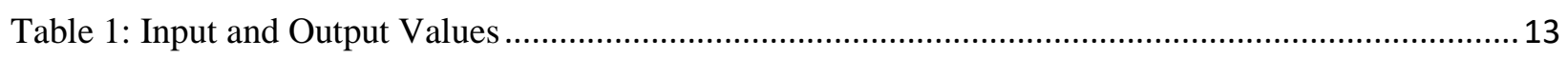




\section{Literature Review}

\section{Marcellus Shale}

The Marcellus Shale is estimated to contain over 140 trillion cubic feet of recoverable natural gas. The Marcellus play has developed into the largest natural gas play in the U.S. producing an average of 13 billion cubic feet per day and providing more than 15\% of the natural gas supply in the U.S in year 2014 . This is all capable with the application of horizontal wells and hydraulic fracturing. After 2005, production rapidly increased to the point of becoming a major source of gas for the eastern U.S. The Marcellus Shale play spans four states: Ohio, Pennsylvania, West Virginia, and New York. The depth ranges from 4,000 to 7,000 feet and primarily produces gas but becomes more liquid-rich in less thermally mature areas of western Pennsylvania. The target zone is anywhere from 50 to 200 feet thick and typically has a porosity that ranges between 5\% and 14\%. (Izadi, Junca, \& Cade, 2014)

The Marcellus shale is one of the largest unconventional gas shale plays in the United States. It underlies much of Pennsylvania, West Virginia, and New York State and even extends under Lake Erie and into Canada. An effective method to stimulate these reservoirs is to horizontally drill into the formation and use hydraulic fracturing. The hydraulic fracturing creates pathways for the hydrocarbon to flow from the shale into production wells. These lateral wells are typically completed from toe to the heel over a number of stages using plug-and-perf method. If a multiple well pad is being stimulated then a zipper-frac style is utilized. The spacing of the wells is designed with a certain dimension of fracture in mind. Companies try to maximized stimulation while minimizing the costs associated with the volume of fluids pumped into the formation and time associated with the treatment. To predict fracture geometry the try to calculate it from factors like the changing stress distributions due to the injections and lithological characteristics of the reservoir, including the presence of natural fractures. Microseismic monitoring is one of the few techniques that highlight the influence of the fracture on the reservoir. The 
fracturing generates sources of microseismic energy that propagate elastic waves through the rock, these waves when recorded by a properly calibrate array of geophones can accurately locate these sources in time and position. (Baig, Urbanic, Goldstein, \& ESG Solutions, 2012)

The energy demand in the United States and internationally is increasing exponentially. The production of natural gas is gaining importance since it is considered to be a cleaner source of energy. There are many natural gas plays across the U.S. with the Marcellus shale play being the largest resource. The Marcellus plays a vital role in the future of gas production and covers several states in the Eastern U.S.. An unknown for the Marcellus shale is the lack of commercially available information. The information gained on the wells and completion designs is rather vague and tightly lipped of kept secret. How the wells are completed plays a significant role in how the production of the shale wells will go. IF a well is not completed properly the well could not be economically feasible to produce. The Marcellus shale has tons of potential in this developing play. From the study, it was found that to be economically feasible to produce the wells need to be horizontal and not vertical wells. It was found though the vertical well could be feasible in a higher gas pricing market. The horizontal wells were not cost efficient to drill longer lateral if the number of fractures placed in the lateral are kept the same. Overall in the Marcellus shale the more money spent up front with larger fracture jobs appeared more economical. (Schweitzer \& Bilgesu, 2009)

\section{Completions}

To increase the productivity of a play in the Marcellus Shale it is crucial to use the appropriate completion and stimulation design. To optimize the treatment design it is important to have a thorough understanding of the rock properties and reservoir characteristics of the gas condensate area and the behaviors of the well during the stimulation of the well. The paper being reviewed looks to optimize the completion design by looking into the well spacing, lateral length, azimuth, and hydraulic fracturing properties that improve the wells productivity. They applied multivariable statistical analysis in conjunction with production analysis to understand the relationships between rock properties and 
hydraulic fracturing efficiency within the reservoir. In the Marcellus Shale, fifty percent of the area of interest is composed of clay shales that are anisotropic in strength and deformability and the brittle failure characteristics vary from east to west. This can affect the proppant transport and ultimately the productivity of the well. A rich data set of 53 wells included well logs, microseismic data and all drilling completion, and hydraulic fracturing data to complete this task. They used production analysis, multivariable statistical analysis, rate transient analysis, mapping and fracture modeling. The multivariate analysis identified the factors most influencing production. The crucial factors were not identical in the east to west parts of the field. The rate transient analysis calibrated to microseismic data yielded important insights relating EUR to well length. Lastly, geomechanics and rock properties were used in hydraulic fracture modeling to show that small changes in fracture design can improve stimulated reservoir volume. (Izadi, Junca, \& Cade, 2014)

Operators typically use geometric perforation designs in order to prepare for hydraulic fracturing which allows them to complete the Marcellus shale's horizontal wells simply and cost-effectively. The perforation clusters are placed at equidistant points along the lateral, but microseismic monitoring shows that this type of stage selection often distributes hydraulic fracturing treatments unevenly. The fracture treatments propagate to the lowest-stress intervals, leaving a large number of perforations under stimulated or even unstimulated. A study is performed to attempt to see which wells using an engineered perforation design compared to wells that have a geometric perforation produce the best results. Based on the initial flow tests and the difference in treating data, it appears that using a fully engineered completion design, driven by measurements taken along the lateral can show positive impacts both operationally and financially. (Walker, et al., 2012)

The completion designs for hydraulic fracture stimulations are created to optimize the spacing of wells and perforation clusters such that the largest volume of the reservoir can be accessed through the promotion of a discrete fracture network in the reservoir. The costs of pumping proppants and fluids down wells are also sought to be minimized as much as possible to get the best result. It is also desirable to minimize the overlap of treatment volumes between neighboring wells and stages to avoid the 
preferential diversion of proppants and fluids into the previously stimulated volumes of the reservoir. When these stimulations are monitored from multiple geophone arrays surrounding the treatment zone, seismic moment tensor inversion (SMTI) analysis offers the ability to test this hypothesis by inferring if the microseismic events are related to the opening of or closure of pre-existing natural or newly created fractures. Hydraulic fracture treatments can activate different fracture sets even within the same pad. Seismic moment sensors can show on microseismic events recorded during treatment of two wells on the same pad, how sub-horizontal fractures corresponding to bedding planes are the dominant activated fractures for one side of a pad while on the other side of the pad, strongly dipping joint sets are facilitating the growth of the fracture. The differences in fracture style indicate that the local stress conditions can be quite heterogeneous across the pad and can have important consequences for the assessment of stage spacing. (Baig, Urbanic, Goldstein, \& ESG Solutions, 2012)

The report being reviewed looks to study a technique known as neural network (ANN) modeling to develop a predictive model to identify performance drivers and evaluate completion effectiveness. Sensitivities performed on this model indicate that well to well variation in reservoir quality and geology has a dominate effect on the Marcellus production. Parameters such as fracture spacing, frac volume, perforation distribution, proppant amount and fluid volume also affect well production. (Shelley, Nejad, Guliyev, Raleigh, \& Matz, 2014)

The geology, reservoir, completion, and hydraulic fracturing all factor into a well's production and recovery capabilities or potential. The use of hydraulic fracturing and reservoir simulation tools are common method to understand what drives well production and model fluid flow inside the reservoir. For the simulations tools, much information about a wells geomechanic characteristics are necessary to build the models to evaluate completion effectiveness. This information includes but is not limited to permeabilities, porosity, saturations, pressures, fluid properties, rock properties and thermodynamics. This data is usually not available so it must be educational predicted or assumed to try and forecast well production. Thousands of models are constructed based on the database in order to evaluate different ways of matching the field data. A representative model can then be chosen based on train, test and 
validation data error, engineering common sense and scrutiny. To improve the process it is important to understand the relationships between inputs and outputs in the model. The data driven approach, unlike traditional discrete modeling, provides a high level perspective and is not biased due to assumptions made early in the modeling process. An ANN model can be created to use readily available information which increases its utility. (Shelley, Nejad, Guliyev, Raleigh, \& Matz, 2014)

A study has been done to identify what effect fracture half-length and well spacing have on the efficiency of gas production and gas recovery from the Marcellus Shale horizontal wells. A commercial reservoir simulator was utilized to develop a base model for the Marcellus Shale formation. The models were constructed using information from the Marcellus Shale Energy and Environment Laboratory (MSEEL). The MSEEL is a research collaboration between West Virginia University, Ohio State University, The National Energy Technology Laboratory, and Northeast Natural Energy. The MSEEL is a long-term laboratory and field study that will study the hydrocarbon production potential and the environmental and economic impacts of drilling and producing well in the Marcellus Shale play. The base model that was used incorporated many of the complex reservoir characteristics that can be associated with the Marcellus Shale play. The reservoir properties, such as porosity, permeability, natural fracture spacing and others were determined using history matching. Data from the two original wells provided by the MSEEL were utilized to history match the daily and cumulative gas production. The impact of the completion parameters on gas recovery was investigated using history-matched models to evaluate the impact that fracture half-length and well spacing have on production. In conclusion to this study, the production histories of both horizontal wells were successfully matched with the models. The results from the simulation indicate that a significant amount of gas remains beyond the drainage areas of the two existing wells. The fractured half-length was found to have a significant impact on the stimulated reservoir volume, gas production, and gas recovery. An efficient completion design can extend the drainage volume as well as eliminate the need for infill drilling. Lastly, simulations show that fracture half-lengths between 450 to 475 feet for the existing wells would have drained the reservoir efficiently, making infill drilling unnecessary. (Filchock, Aminian, \& Ameri, 2016) 
Advances in hydraulic fracturing and horizontal well technology have unlocked considerable reserves of hydrocarbon contained in shale formation. But, quantifying key shale petrophysical properties still remain challenging. Using standard sate techniques to measure the permeability of unconventional formations such as the Marcellus Shale play is not practical. The permeability of the shales are typically values in nano-Darcy range so unsteady state methods must be utilized to estimate the permeability. However, these techniques suffer from a large margin of uncertainty and reproducibility problems. The problems can be associated to the lack of consistent experimental protocols and the interpretations of the transient data. The experiments cannot be performed under the reservoir stress and temperature which limits the unsteady-state measurements. To attempt to obtain more accurate and trustworthy data, core plugs will be tested using a fully automated laboratory set-up. From these experiments they will evaluate the ultra-low permeability pertrophysical properties under the confining pressure. The permeability of the core plug were first measured under different gas pressure at constant net stress. Then the absolute permeability was determined by applying the gas double-slippage correction. The porosity of permeability of the core plug were then measured under a wide range of net stress. From these methods it was found that the permeability and porosity can be successfully measured under a wide range of pressures in the Marcellus Shale. Consistent and reliable value for the shale absolute permeability cab be obtained by the application of the gas double-slippage correction to measure permeability values at different gas pressures. With an increase in net stress the permeability and porosity of the shale decrease. Pressures at which the fractures fully close can be successfully determined by the Walsh plot. Permeability and porosity exhibit non-linear results with the difference in the compressibility of the fracture and matrix system. The fracture system accounts for as much as $50 \%$ of the total permeability but only up to $12 \%$ of the total porosity. A reduction in the absolute permeability can be attributed to adsorption of Carbon Dioxide to the Marcellus shale sample. (Zarmirian, Aminian, \& Ameri, 2016)

A key technology to complete horizontal wells in the shale gas reservoirs is multi-stage hydraulic fracturing. Multiple perforation clusters are used to create multiple transverse fractures in east stage. Both short and long-term production performance is affected by how these clusters are placed. Previous 
work demonstrates that when more than two fractures are created, mechanical interaction among fractures creates strong stress concentrations around the inner fractures. The fractures between the two edge factors are limited to dilate and their widths are much less than the edge-fractures' width. By quantitatively incorporating the author's previous work reservoir simulation models were created to investigate the impacts of the number of perforation clusters and cluster spacing on production performance of horizontal shale gas wells. With the cluster spacing kept the same and more clusters added, gives a lower ultimate gas recovery because of the increased number of less effective inner fractures. Reducing the cluster spacing increases the total number of fractures, but doesn't necessarily improve the production of the well. Lower cluster spacing can actually lead to more less-effective and ineffective fractures and give a lower gas rate and recovery. (Cheng, 2010)

\section{Production Logging}

Production logging denotes that area of a well logging concerned with two general goals of problem well diagnosis and reservoir surveillance. The purpose of logging is to track fluid movement within or behind pipe or to monitor the movement of reservoir fluid contacts. Logging has become vital to drilling engineers to maintain safe work conditions with increasing hazards of drilling. In most cases, a production log is obtained before a well is perforated for production. The production log is ran to obtain desired information that can assist and even improve the production and safety of a well. (McKinley, 1982)

The spinner flowmeter consists of a propeller mounted on a jewel supported shaft. These devices are stopped in the perforation-free section of the casing, the packer element inflated, and a measurement taken. They are excellent for use when the flowrate has a range of 15-2000 BPD. The interference of these tools with normal flow, their inability to measure high flow rates, or to profile in perforated intervals in addition to the operational inconvenience brought about the popularity of the non-diverting or continuous spinner. The continuous spinners log continuously at a constant logging speed. They are run centralized and sample only a portion of the flow field. (McKinley, 1982) 
There is not a generic difference between a continuous and fullbore spinner. The fullbore spinner folds into a diameter no greater than that of the tool when in the tubing, but expands into a larger diameter for surveying in the casing. The continuous spinner does not have that capability, but the difference between the two is too small to justify a separate discussion of each. They both share three common features. First, the spinner element on each is at the very bottom of the tool string. Second, the presence of at least two centralizers in any tool string containing the spinner flowmeter. These centralizers ensure that the spinner element samples the same location in the wellbore's cross section at each depth. For calibration purposes this consistency is necessary. Thirdly, both tools use a four-blade propeller-type spinner element. The spinner element is free to rotate either clockwise or counterclockwise depending upon the movement of the fluid relative to the barrel of the tool. Both continuous and fullbore tools are intended for quantitative use only in flow streams having a single component of velocity directed along the axis of the tool. The stream should be a single-phase or a high-rate multiphase. In the multiphase case, the well should have a low deviation angle. (Continuous and fullbore spinner flowmeters, 2017)

The continuous tools are available in a wide range of configurations, more so than the fullbore tools. The centralizers also come in a variety of configurations. Power centralizers offer the least problem to entry through tubing. These are closed by strong springs when in the tubing. In the casing, a downhole motor opens the centralizer against the spring force. In case of a failure downhole by the motor, the powered centralizer has a shearing mechanism so that the constriction at the tubing's end can be used to close the centralizer when re-entering the tubing. Some continuous tools have the spinner element inside the bow-spring cage with no additional protection from damage. Most of the continuous spinners are rated for pressures in the range of 15,000 to 20,000 psi and temperatures of 350 to 400 degrees Fahrenheit. Some tools can accommodate 500 degrees Fahrenheit, but they employ vacuum flasks and thus have a diameter of at least 2.5 inches. (Continuous and fullbore spinner flowmeters, 2017)

Most logs are used to characterize the wellbore, formation, and fluids prior to well completion, but there are a number of logging tools that can provide information during the production of the well and beyond. These types of logs are considered production logs. Production logs are used to allocate 
production on a zone by zone basis and also to diagnose production problems such as leaks or cross flow. It would be ideal to measure radial inflow rates using a cheap and accurate sensor, but no such sensor exists. The alternative would be to measure the axial flow rate in a well at a depth above and below the zone of interest and compute the difference and hence the inflow rate, but there is not any practical measurement of axial flow rate beyond some special applications of oxygen activation logs. It is possible to measure an axial velocity and combine this with an assumed or measured internal diameter to arrive at an axial flow rate. Some common velocity sensors include; turbine/spinner flowmeters, markers/tracers such as oxygen activation logs or radioactive iodine tracer logs, and heated anemometry. The Spinners are assumed to rotate at a speed proportional to the average fluid velocity passing through the swept area of the blades with an offset for friction and imperfections. This is a simple gain and offset transformation from the rotational speed of the spinner. A downside is that the gain and offset are not constants but are a complicated function of fluid density, fluid viscosity, spinner pitch, pipe diameter, fluid velocity, and more. The spinner is typically calibrated downhole by recording the spinner speed at a series of different logging speeds and plotting the resultant average spinner speed versus the corresponding average logging speed to determine the slope (gain) and threshold (offset). Next, the calibrated spinner velocity needs to be converted to an average pipe velocity. The correction coefficient determined by the velocity profile across the pipe cross section can vary from 0.5 for an infinitely small spinner in laminar flow to 1.0 for a spinner that sweeps the entire pipe area. (Production logging, 2017)

From the time the well is drilled until it is abandoned the production logging tools find many applications. An appropriate categorization of production logs is by usage. This approach leads to five distinct categories and also represent a rough chronological order of tool evolution. Category one are tools that are used to track movement of fluid either inside or immediately outside the casing of a well. Category two are tools that perform cement-placement monitoring. Category three are tools that deal with zonal isolation when pressure imbalance causes crossflow through poorly cemented sections, leading to excessive production of unwanted fluids. Categories four and five are tools for monitoring fluid contacts in formations and selection of recompletion zones. (Production logging, 2017) 
There are three common misconceptions about production logging. The first is that a production $\log$ can be run by anyone. The second is only one logging tool is needed. Lastly, the answer or anomaly will jump out from a casual scan of the log. Quality control is important and careful attention must be focused upon procedure, tool calibration, and depth control while running a production log. To obtain an accurate production log an experienced and knowledgeable operator must perform the task. Production logging tools should be run in complementing suites similar to openhole logging so that one log can be compared with another. Very seldom will a single log show a problem sufficiently to prescribe a remedial action. The logs should be interpreted in a consistent fashion that first identifies normal or expected features. The abnormal portions can be then be examined to determine which parts are pertinent to the problem and which parts are irrelevant. (Production logging, 2017)

Production logging is a term that covers a wide array of sensors, measurements, and interpretation techniques. Production logs are used by the operators to evaluate fluid movement in and out of wellbores, quantify flow rates and determine fluid properties at downhole conditions. Completion engineers can evaluate production and perforation efficiency and plan remediation or modify future completion designs based on the interpretation of production logs. Reservoir and production engineers can use the production logs to help manage and optimize well and reservoir performance. Since the 1930's production logging has been around. In the beginning they measured wellbore temperature and over time began to measure pressure, fluid density, flow velocity, and holdup. A comprehensive production log evaluation must be performed from downhole. Fluid velocity is commonly measured with a spinner flowmeter which consists of a rotating blade that turns when fluid moves past it. The rotational speed of the bade in revolutions per second is proportional to the fluid velocity with ideal conditions. Friction in the spinner bearings and effects from fluid viscosity result in nonlinear velocity responses, requiring calibration of the measurement. Calibration of the spinner flowmeter is done by making upward and downward passes at varying logging speeds. The spinner speed must be corrected for relative tool speed before absolute fluid velocity. The absolute fluid velocity is not the same as the average velocity due to friction near the pipe 
wall. Engineers convert the spinner velocity to an average velocity by applying corrections factors and using computer modeling techniques. (Parijat, 2013)

\section{Objective}

The goal of this research is to see if there is a trend between the completion procedure used and the amount of gas produced per each stage of well $3 \mathrm{H}$ operated by Northeastern Natural Energy. If a trend can be discovered it will assist in the future planning of the procedure used to complete other wells in this reservoir. The neural network fitting application in Matlab will be the software that is utilized to attempt to reach this objective.

\section{Methodology}

The first step to achieving the objective of finding if a trend between the data exists is to collect the available data. Completion data was collected from Northeastern Natural Energy via MSEEL (Marcellus Shale Energy and Environment Laboratory). MSEEL is collaboration between Northeastern Natural Energy, Department of Energy and West Virginia University to study and try to better understand the Marcellus play in this area. Along with all the completion data for each stage there was also the gas produced information provided. With both of these I have the inputs used to complete the well and the output of the gas that was produced from each stage. There were a total of 28 stages in this well, but for this research only stages 2 through 28 will be studied due to missing information within the first stage of the well.

Once all the available and appropriate data was collected I then had to organize the information to be utilized in the Matlab software. From the data collected I had 10 inputs of 27 samples each and 1 output of 27 samples. The 10 inputs included cluster count, plug depth, total shots, natural fractures, measured slurry, measured clean fluid, measured proppant, pump time and stage length. The output was the surface gas flow rate that was recorded per each of the 27 stages. In Matlab I decided to use the 
Neural Network Fitting application to test the data for a possible trend. When using this application I ran several tests varying all possible parameters which will later on be discussed in more detailed. From each run I recorded the results and the parameters used to achieve thus results. After all possible runs were completed and recorded I then when through the information to find the best parameters to test the possible trend between the inputs and surface gas flow rate.

\section{Discussion}

For this research, data was obtained from a Northeast Natural Energy well named 3H located in Morgantown, West Virginia. The information gathered consists of: cluster count, plug depth, total shots, natural fractures, measure slurry, measured clean fluid, measured proppant, pump time, stage length and surface gas flow rate._ My goal was to see if the completion procedure used had a trend with the surface gas flow rate that was reported. To solve this I decided to use Matlab's Neural Network Fitting application. To use the Neural Network Fitting application an input and output had to be established. For the inputs I used cluster count, plug depth, total shots, natural fractures, measure slurry, measured clean fluid, measured proppant, pump time and stage length. For the output the surface gas flow rate was used. Since there were a total of 27 stages for this well there was 27 data points for each input and output to see if a trend did exist. After the inputs and output values were into the application the next step was to decide what training $\%$, validation $\%$, testing $\%$ and number of hidden neurons to use. The number of hidden neurons was kept at the default of 10 , because it gave the best regression results. I decided to try every possibility of training, validation and testing percentages which gave me a total of 49 different tests that will all be discussed.

The following table, Table 1, shows all 49 tests that were run and the regression results it produced. I will also go over the plot regressions for each of the 49 runs also. 
Table 1: Input and Output Values

\begin{tabular}{|c|c|c|c|c|c|c|c|c|}
\hline \multicolumn{5}{|c|}{ INPUTS } & \multicolumn{4}{|c|}{ OUTPUTS } \\
\hline \begin{tabular}{|l|} 
Figure \# \\
\end{tabular} & Training \% & Validation \% & Testing \% & Hidden Neurons & Training R Value & Validation R Value & Test R Value & All R Value \\
\hline 1 & $90 \%$ & $5 \%$ & $5 \%$ & 10 & 0.999950 & 0.000000 & 0.000000 & 0.999830 \\
\hline 2 & $85 \%$ & $5 \%$ & $10 \%$ & 10 & 1.000000 & 0.000000 & 0.992300 & 0.992130 \\
\hline 3 & $80 \%$ & $5 \%$ & $15 \%$ & 10 & 1.000000 & 0.000000 & 0.972970 & 0.994280 \\
\hline 4 & $75 \%$ & $5 \%$ & $20 \%$ & 10 & 0.999800 & 0.000000 & 0.997150 & 0.988520 \\
\hline 5 & $70 \%$ & $5 \%$ & $25 \%$ & 10 & 1.000000 & 0.000000 & 0.851080 & 0.951950 \\
\hline 6 & $65 \%$ & $5 \%$ & $30 \%$ & 10 & 0.999990 & 0.000000 & 0.960590 & 0.989030 \\
\hline 7 & $60 \%$ & $5 \%$ & $35 \%$ & 10 & 1.000000 & 0.000000 & 0.993550 & 0.994570 \\
\hline 8 & $85 \%$ & $10 \%$ & $5 \%$ & 10 & 0.997230 & 0.987510 & 0.000000 & 0.978540 \\
\hline 9 & $80 \%$ & $10 \%$ & $10 \%$ & 10 & 0.999950 & 0.999550 & 0.986580 & 0.995530 \\
\hline 10 & $75 \%$ & $10 \%$ & $15 \%$ & 10 & 1.000000 & 0.999880 & 0.992770 & 0.990770 \\
\hline 11 & $70 \%$ & $10 \%$ & $20 \%$ & 10 & 1.000000 & 0.977200 & 0.981920 & 0.962050 \\
\hline 12 & $65 \%$ & $10 \%$ & $25 \%$ & 10 & 0.991600 & 0.974570 & 0.992050 & 0.990440 \\
\hline 13 & $60 \%$ & $10 \%$ & $30 \%$ & 10 & 0.991810 & 0.995470 & 0.975200 & 0.980860 \\
\hline 14 & $55 \%$ & $10 \%$ & $35 \%$ & 10 & 0.995380 & 0.999990 & 0.994420 & 0.990370 \\
\hline 15 & $80 \%$ & $15 \%$ & $5 \%$ & 10 & 0.995880 & 0.992290 & 0.000000 & 0.992640 \\
\hline 16 & $75 \%$ & $15 \%$ & $10 \%$ & 10 & 1.000000 & 0.980760 & 0.999990 & 0.996350 \\
\hline 17 & $70 \%$ & $15 \%$ & $15 \%$ & 10 & 1.000000 & 0.959580 & 0.999480 & 0.993670 \\
\hline 18 & $65 \%$ & $15 \%$ & $20 \%$ & 10 & 1.000000 & 0.988730 & 0.984630 & 0.983170 \\
\hline 19 & $60 \%$ & $15 \%$ & $25 \%$ & 10 & 0.967240 & 0.988440 & 0.995610 & 0.973290 \\
\hline 20 & $55 \%$ & $15 \%$ & $30 \%$ & 10 & 0.995830 & 0.958910 & 0.966850 & 0.970820 \\
\hline 21 & $50 \%$ & $15 \%$ & $35 \%$ & 10 & 0.996510 & 0.930550 & 0.935780 & 0.952650 \\
\hline 22 & $75 \%$ & $20 \%$ & $5 \%$ & 10 & 0.999990 & 0.913960 & 0.000000 & 0.980690 \\
\hline 23 & $70 \%$ & $20 \%$ & $10 \%$ & 10 & 1.000000 & 0.995270 & 1.000000 & 0.996670 \\
\hline 24 & $65 \%$ & $20 \%$ & $15 \%$ & 10 & 1.000000 & 0.964800 & 0.998260 & 0.993320 \\
\hline 25 & $60 \%$ & $20 \%$ & $20 \%$ & 10 & 0.998260 & 0.985920 & 0.992960 & 0.980680 \\
\hline 26 & $55 \%$ & $20 \%$ & $25 \%$ & 10 & 0.999550 & 0.987710 & 0.990470 & 0.990970 \\
\hline 27 & $50 \%$ & $20 \%$ & $30 \%$ & 10 & 1.000000 & 0.949720 & 0.992610 & 0.975650 \\
\hline 28 & $45 \%$ & $20 \%$ & $35 \%$ & 10 & 1.000000 & 0.951960 & 0.907670 & 0.962440 \\
\hline 29 & $70 \%$ & $25 \%$ & $5 \%$ & 10 & 1.000000 & 0.942090 & 0.000000 & 0.978000 \\
\hline 30 & $65 \%$ & $25 \%$ & $10 \%$ & 10 & 0.999330 & 0.995690 & 0.998290 & 0.997750 \\
\hline 31 & $60 \%$ & $25 \%$ & $15 \%$ & 10 & 1.000000 & 0.947390 & 0.964220 & 0.971260 \\
\hline 32 & $55 \%$ & $25 \%$ & $20 \%$ & 10 & 1.000000 & 0.986860 & 0.984010 & 0.990630 \\
\hline 33 & $50 \%$ & $25 \%$ & $25 \%$ & 10 & 1.000000 & 0.994390 & 0.982490 & 0.994050 \\
\hline 34 & $45 \%$ & $25 \%$ & $30 \%$ & 10 & 1.000000 & 0.727840 & 0.850040 & 0.898340 \\
\hline 35 & $40 \%$ & $25 \%$ & $35 \%$ & 10 & 1.000000 & 0.716300 & 0.935810 & 0.842670 \\
\hline 36 & $65 \%$ & $30 \%$ & $5 \%$ & 10 & 0.998300 & 0.960890 & 0.000000 & 0.979300 \\
\hline 37 & $60 \%$ & $30 \%$ & $10 \%$ & 10 & 0.999880 & 0.975540 & 0.964850 & 0.990600 \\
\hline 38 & $55 \%$ & $30 \%$ & $15 \%$ & 10 & 1.000000 & 0.914830 & 0.999840 & 0.985480 \\
\hline 39 & $50 \%$ & $30 \%$ & $20 \%$ & 10 & 1.000000 & 0.902980 & 0.990750 & 0.950450 \\
\hline 40 & $45 \%$ & $30 \%$ & $25 \%$ & 10 & 1.000000 & 0.893970 & 0.945920 & 0.967830 \\
\hline 41 & $40 \%$ & $30 \%$ & $30 \%$ & 10 & 0.999870 & 0.957590 & 0.934320 & 0.971750 \\
\hline 42 & $35 \%$ & $30 \%$ & $35 \%$ & 10 & 1.000000 & 0.920700 & 0.974440 & 0.967780 \\
\hline 43 & $60 \%$ & $35 \%$ & $5 \%$ & 10 & 1.000000 & 0.971570 & 0.000000 & 0.991900 \\
\hline 44 & $55 \%$ & $35 \%$ & $10 \%$ & 10 & 1.000000 & 0.941830 & 0.995930 & 0.967680 \\
\hline 45 & $50 \%$ & $35 \%$ & $15 \%$ & 10 & 1.000000 & 0.954880 & 0.996250 & 0.980750 \\
\hline 46 & $45 \%$ & $35 \%$ & $20 \%$ & 10 & 1.000000 & 0.955850 & 0.995820 & 0.969450 \\
\hline 47 & $40 \%$ & $35 \%$ & $25 \%$ & 10 & 0.999940 & 0.975790 & 0.954440 & 0.983190 \\
\hline 48 & $35 \%$ & $35 \%$ & $30 \%$ & 10 & 1.000000 & 0.914460 & 0.983430 & 0.961750 \\
\hline 49 & $30 \%$ & $35 \%$ & $35 \%$ & 10 & 1.000000 & 0.890200 & 0.947060 & 0.936130 \\
\hline
\end{tabular}


From the above table one can see that some of the runs gave better results that the others. Out of the 49 runs the top 3 were found to be run number 10, 16, and 23 .

Validation $\%$ at $5 \%$

The following figures, Figures 1-7, are for the first 7 runs at a $5 \%$ validation. It was found that using only a 5\% validation as an input was too low with only 27 data points for each parameter. The validation $\mathrm{R}$ value was $\mathrm{NaN}$ for these first 7 runs. 
90\% Training, 5\% Validation, 5\% Testing and 10 Hidden Neurons
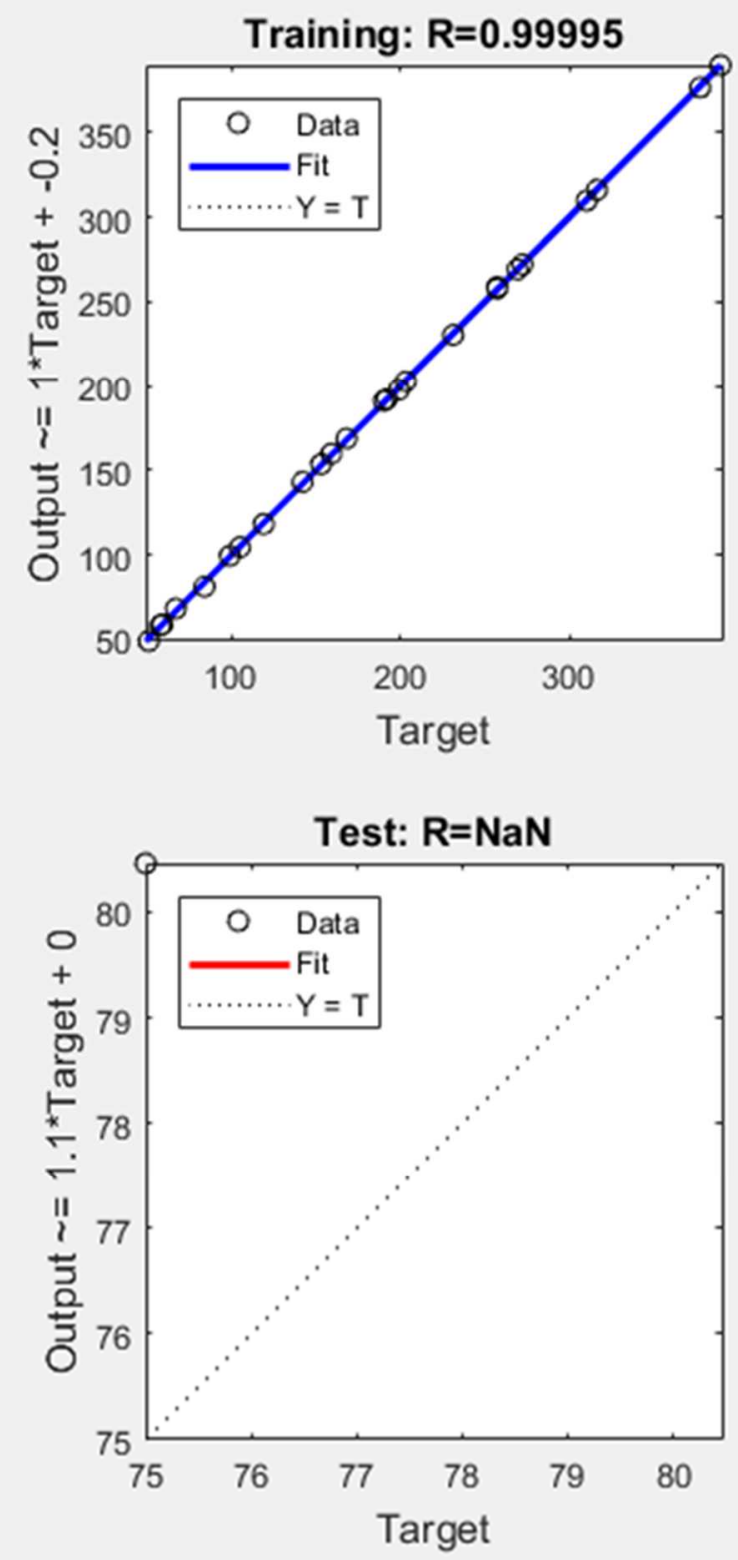

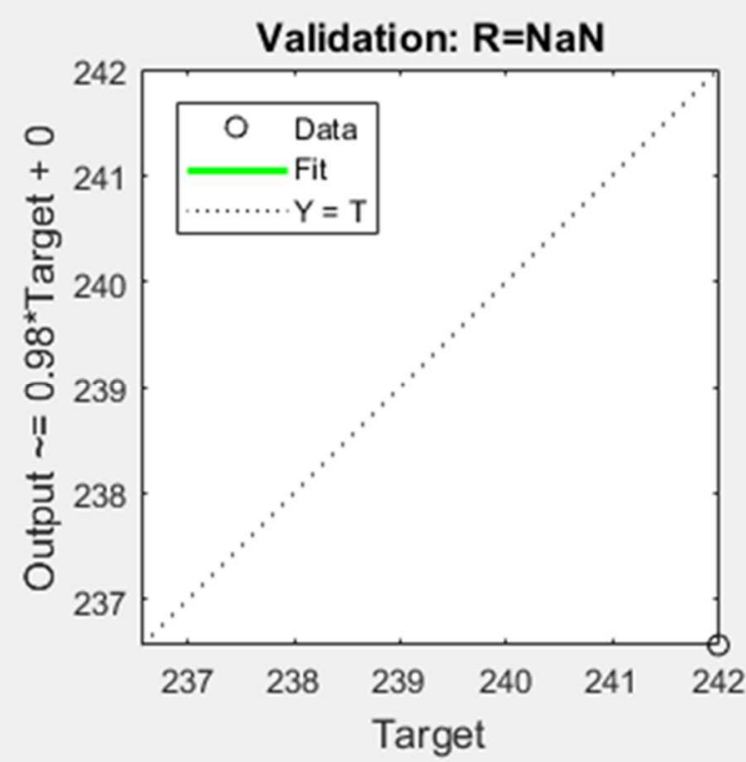

All: $\mathbf{R}=\mathbf{0 . 9 9 9 8 3}$

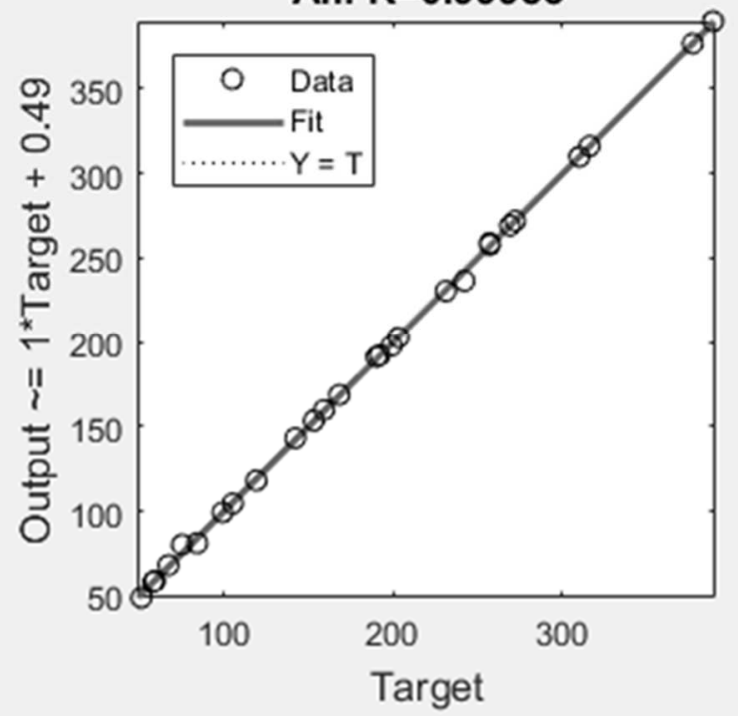

Figure 1: 90\% Training, 5\% Validation, 5\% Testing and 10 Hidden Neurons 


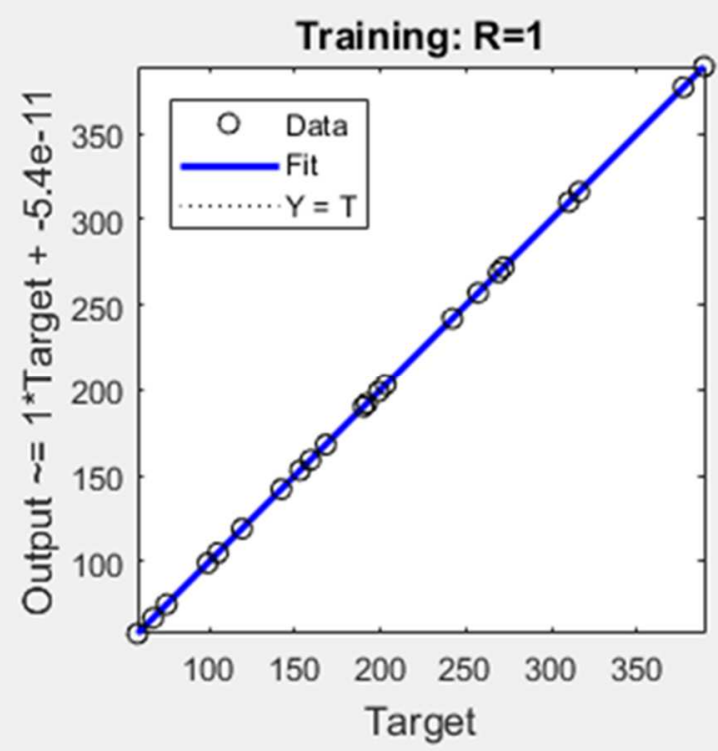

Test: $\mathbf{R}=\mathbf{0 . 9 9 2 3}$

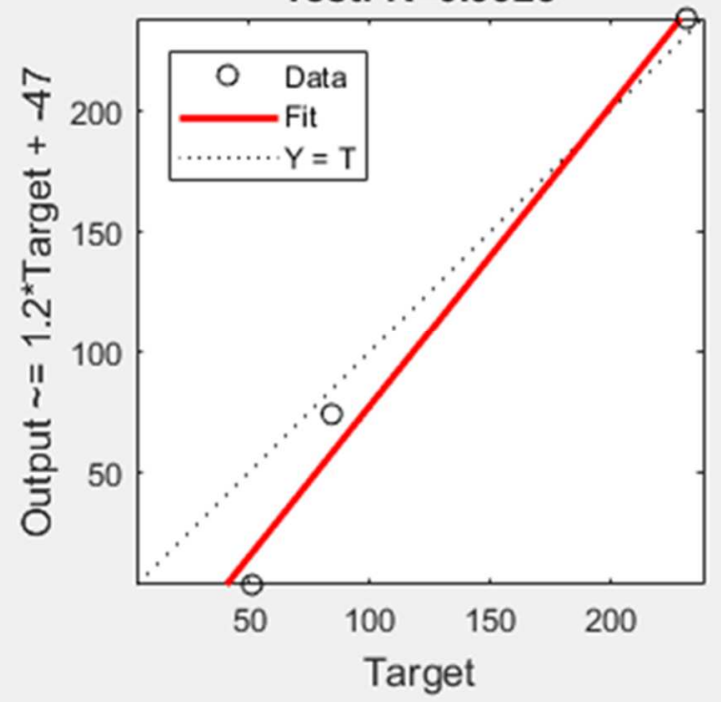

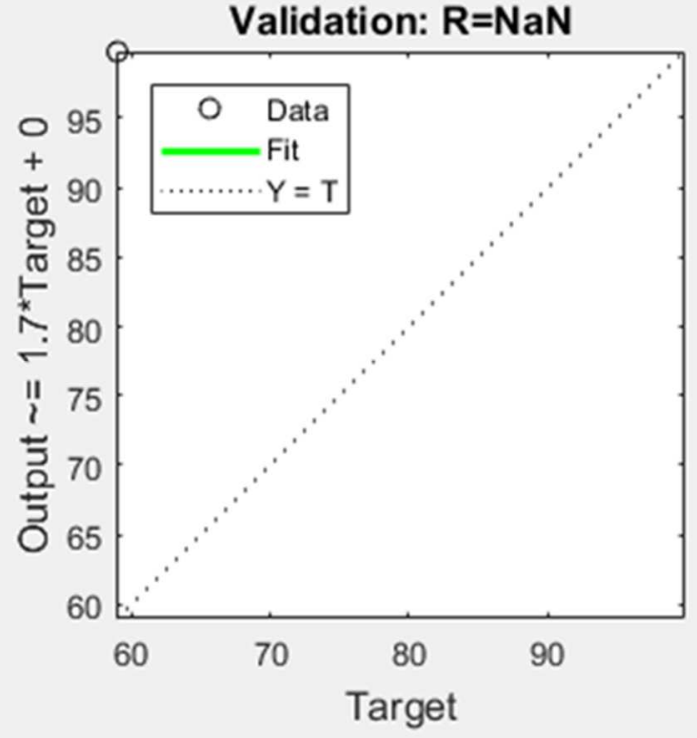

All: $\mathbf{R}=0.99213$

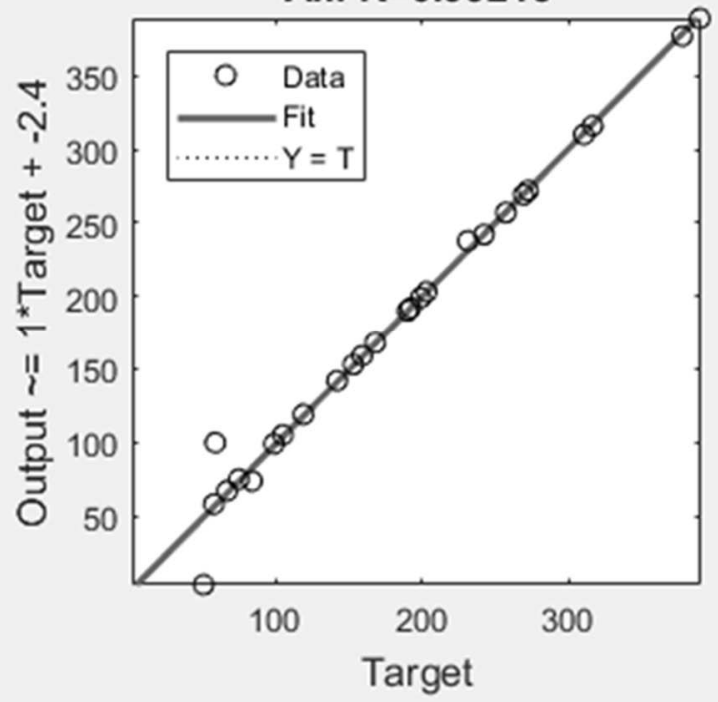

Figure 2: 85\% Training, 5\% Validation, 10\% Testing and 10 Hidden Neurons 

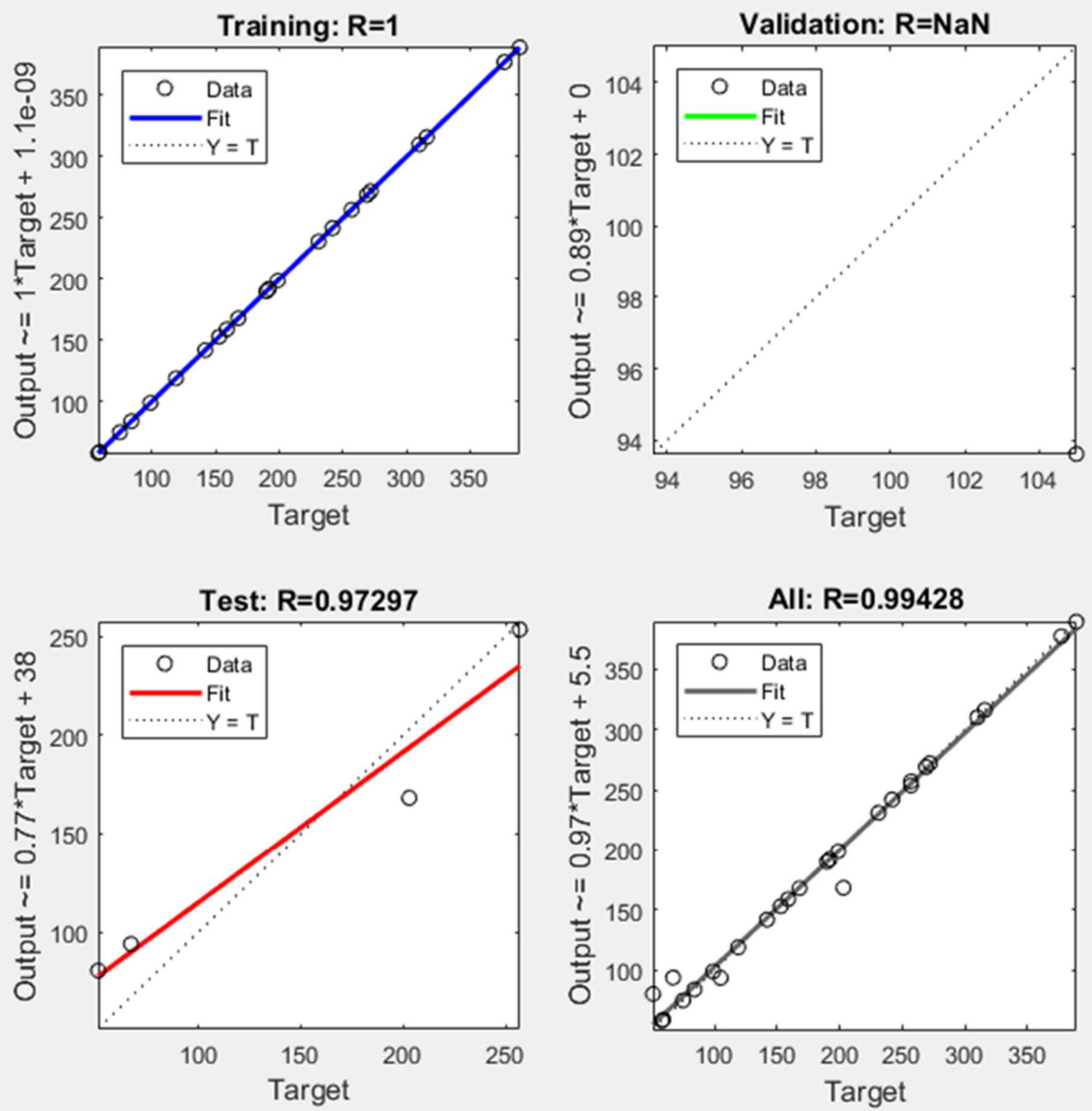

Figure 3: 80\% Training, 5\% Validation, 15\% Testing and 10 Hidden Neurons 

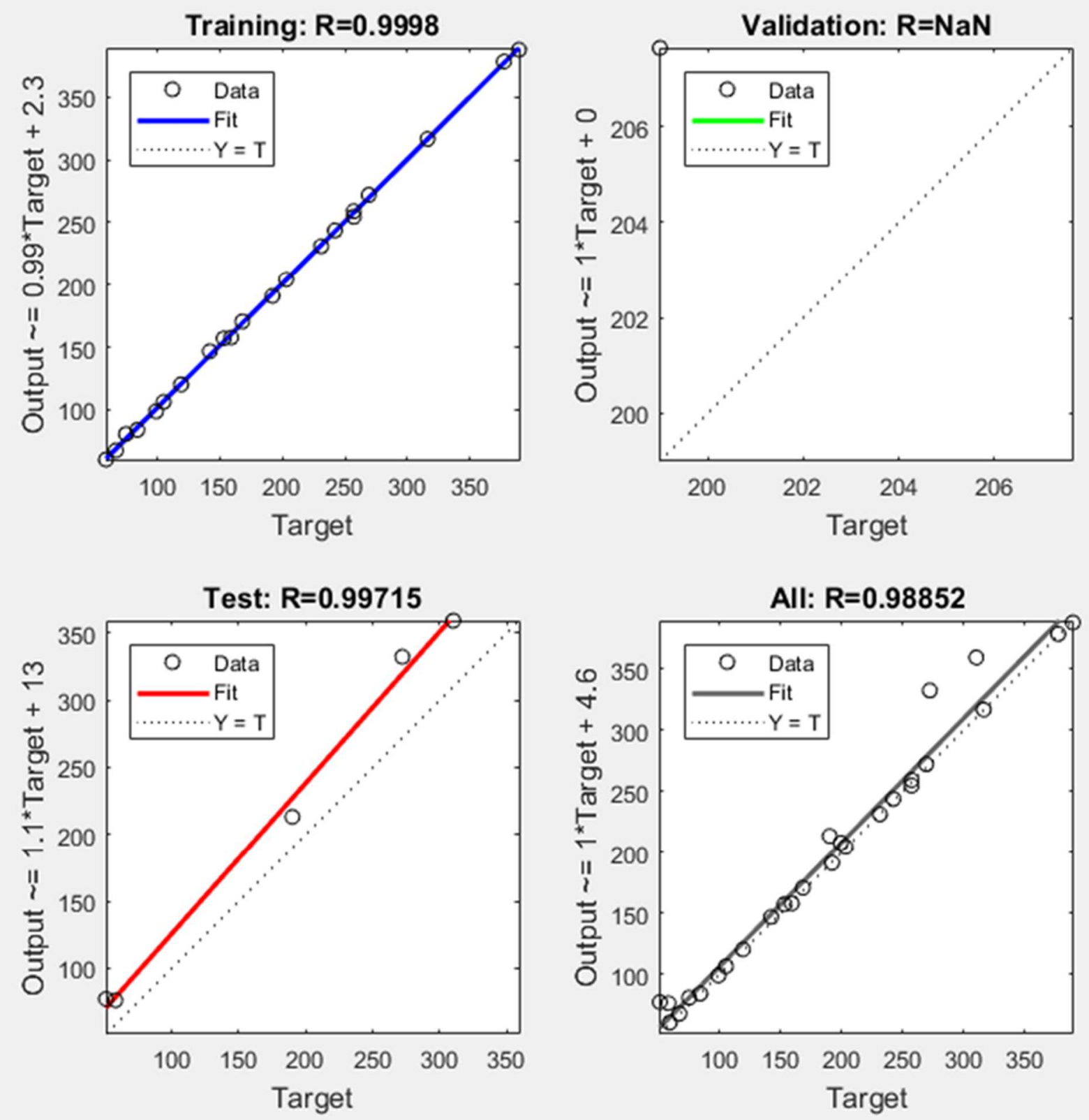

Figure 4: 75\% Training, 5\% Validation, 20\% Testing and 10 Hidden Neurons 

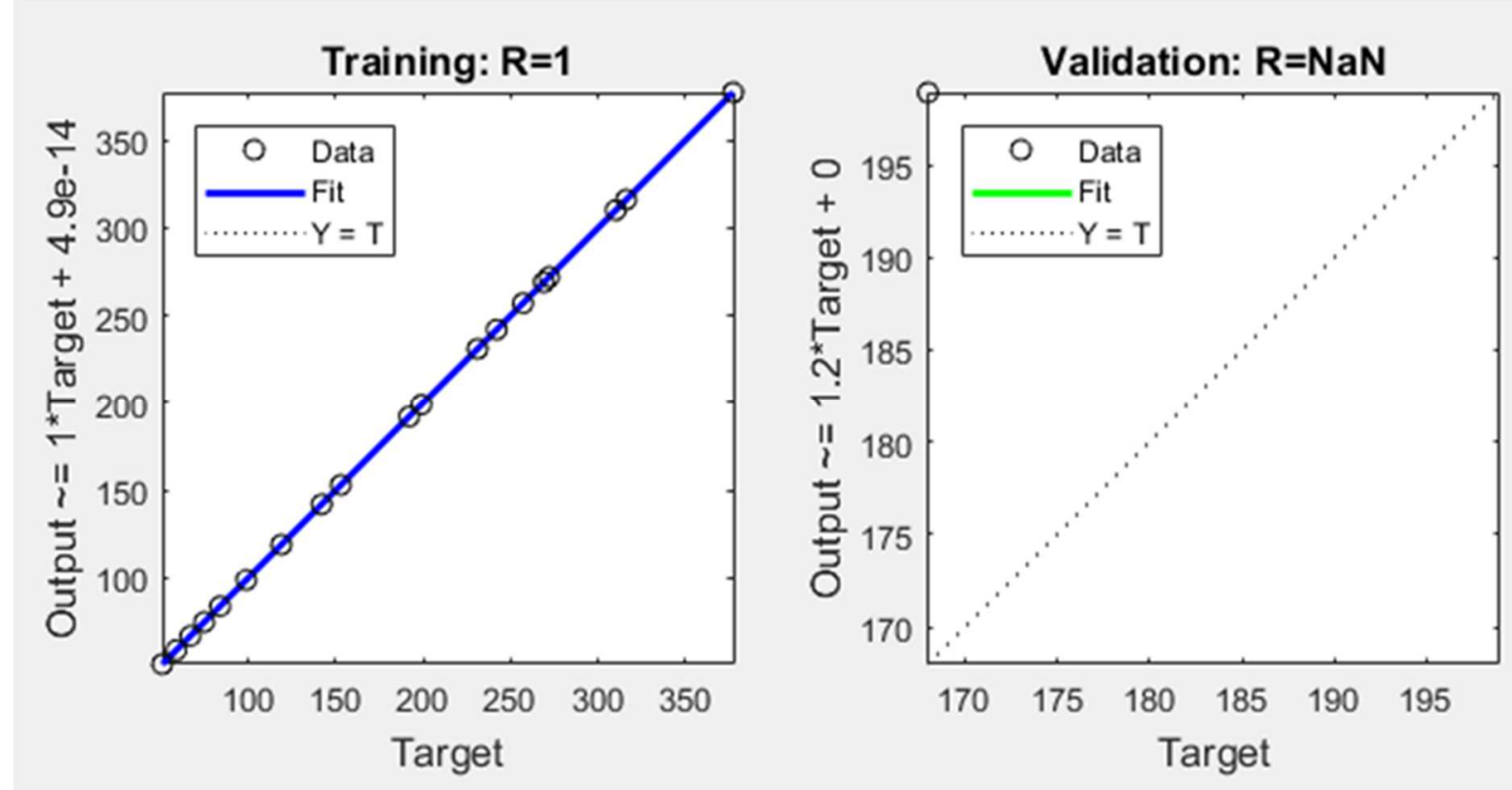

Test: $\mathbf{R}=\mathbf{0 . 8 5 1 0 8}$
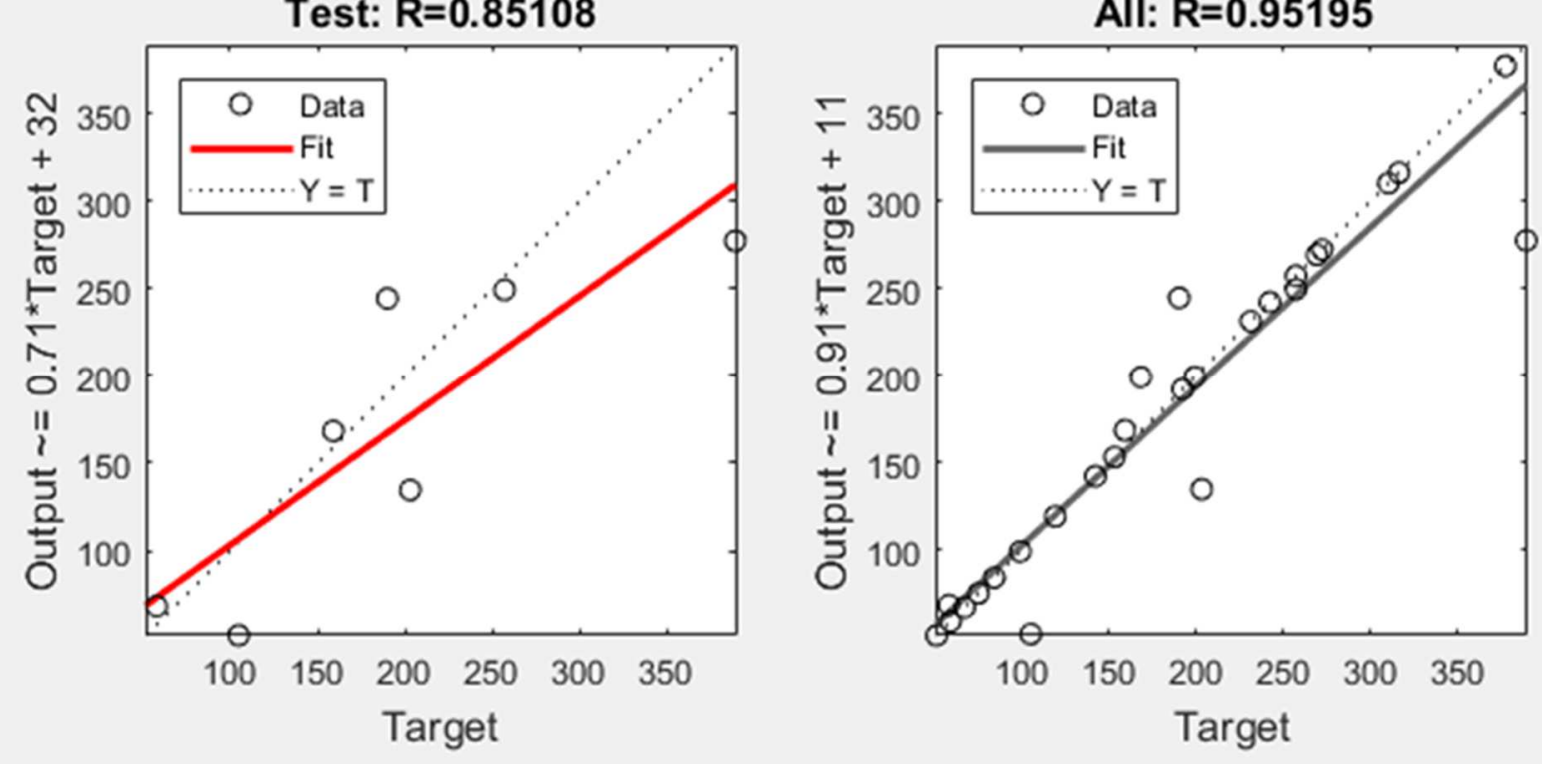

Figure 5: 70\% Training, 5\% Validation, 25\% Testing and 10 Hidden Neurons 

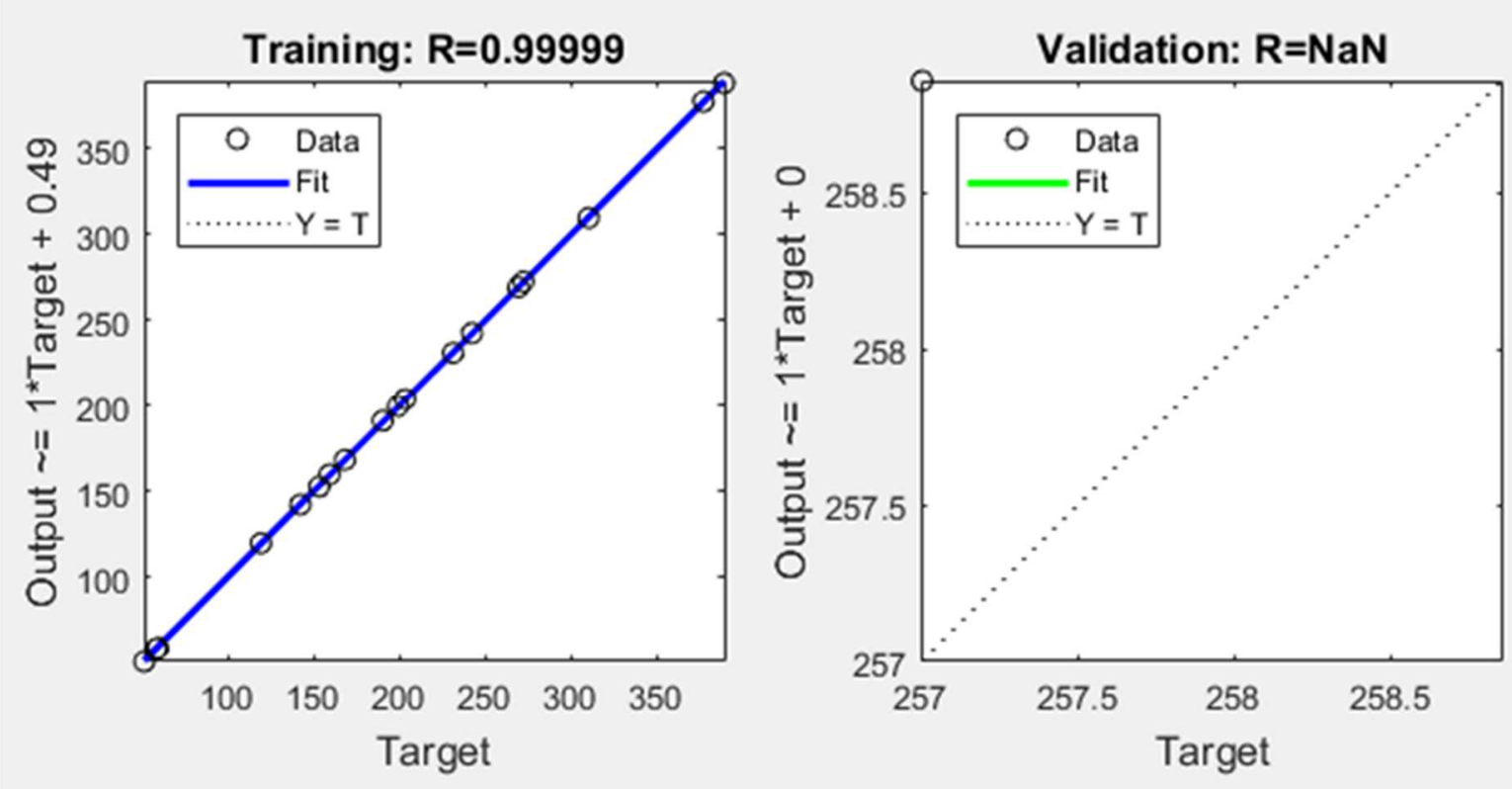

Test: $\mathbf{R}=\mathbf{0 . 9 6 0 5 9}$
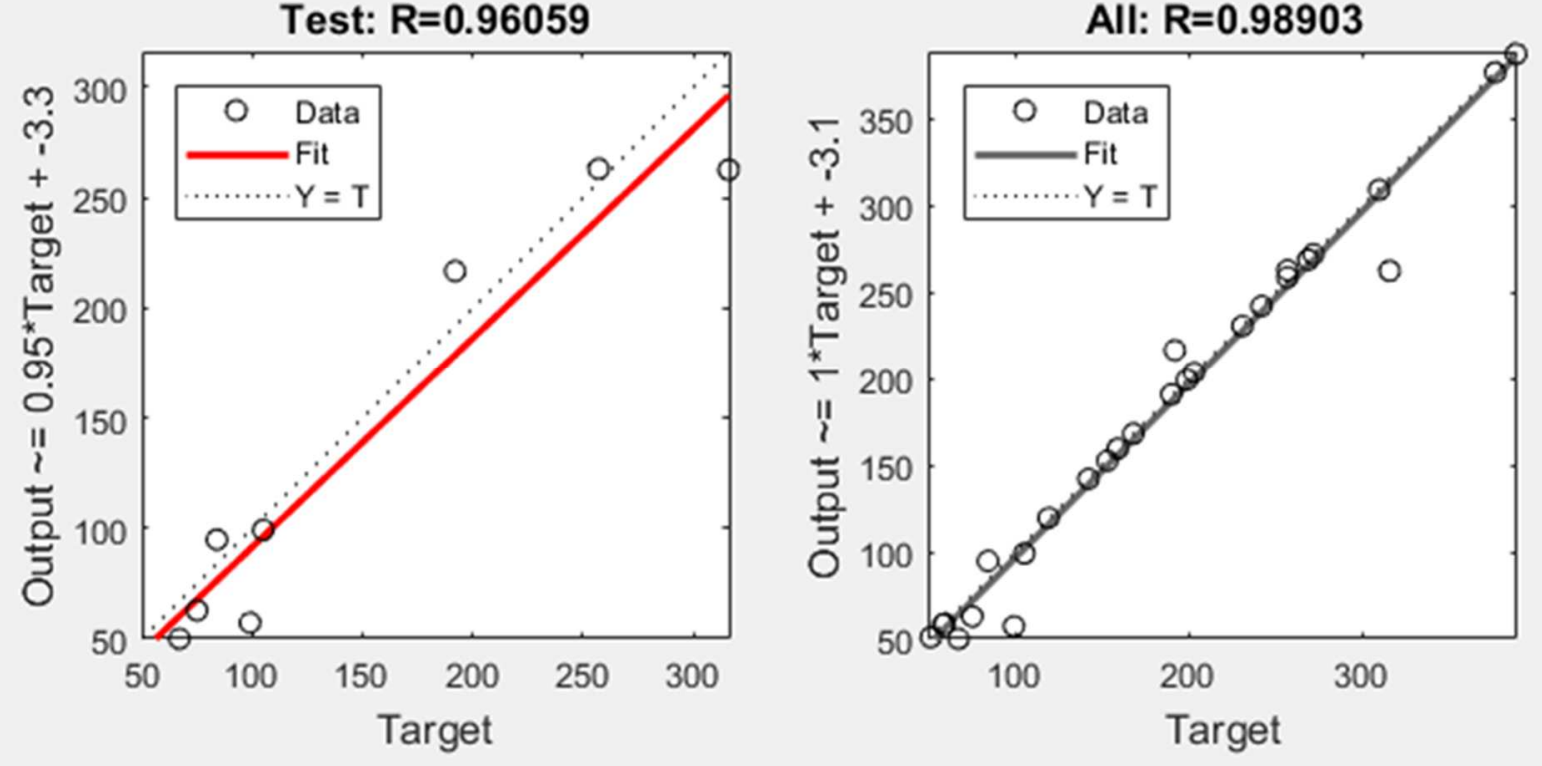

Figure 6: 65\% Training, 5\% Validation, 30\% Testing and 10 Hidden Neurons 

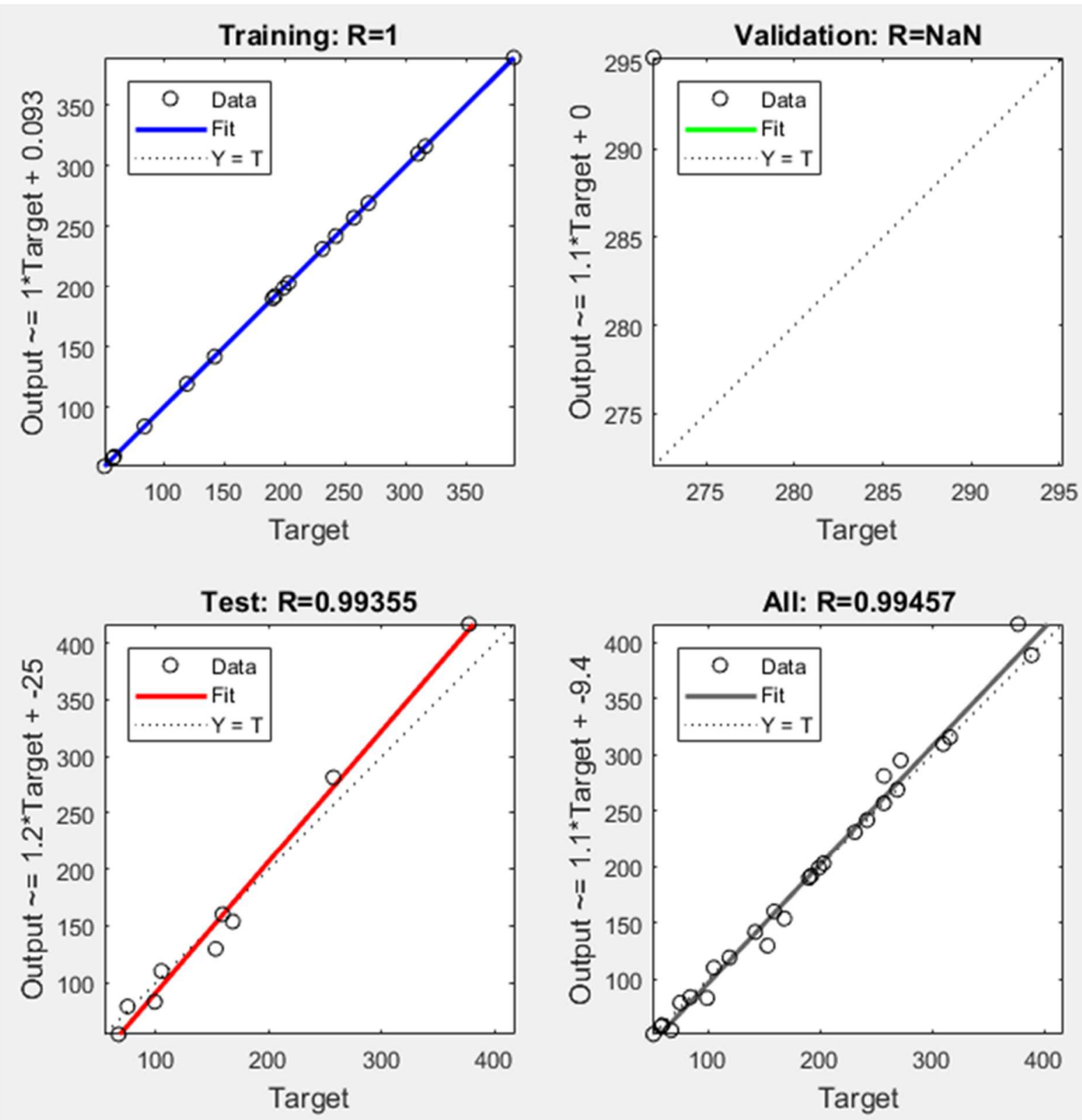

Figure 7: 60\% Training, 5\% Validation, 35\% Testing and 10 Hidden Neurons

Testing $\%$ at $5 \%$

When looking over the results from the regression runs it can also be stated that using $5 \%$ as a testing value was too low and gave a result of $\mathrm{NaN}$ for the testing $\mathrm{R}$ Value. This can be seen in Figures 8, $15,22,29,36$ and 43. 

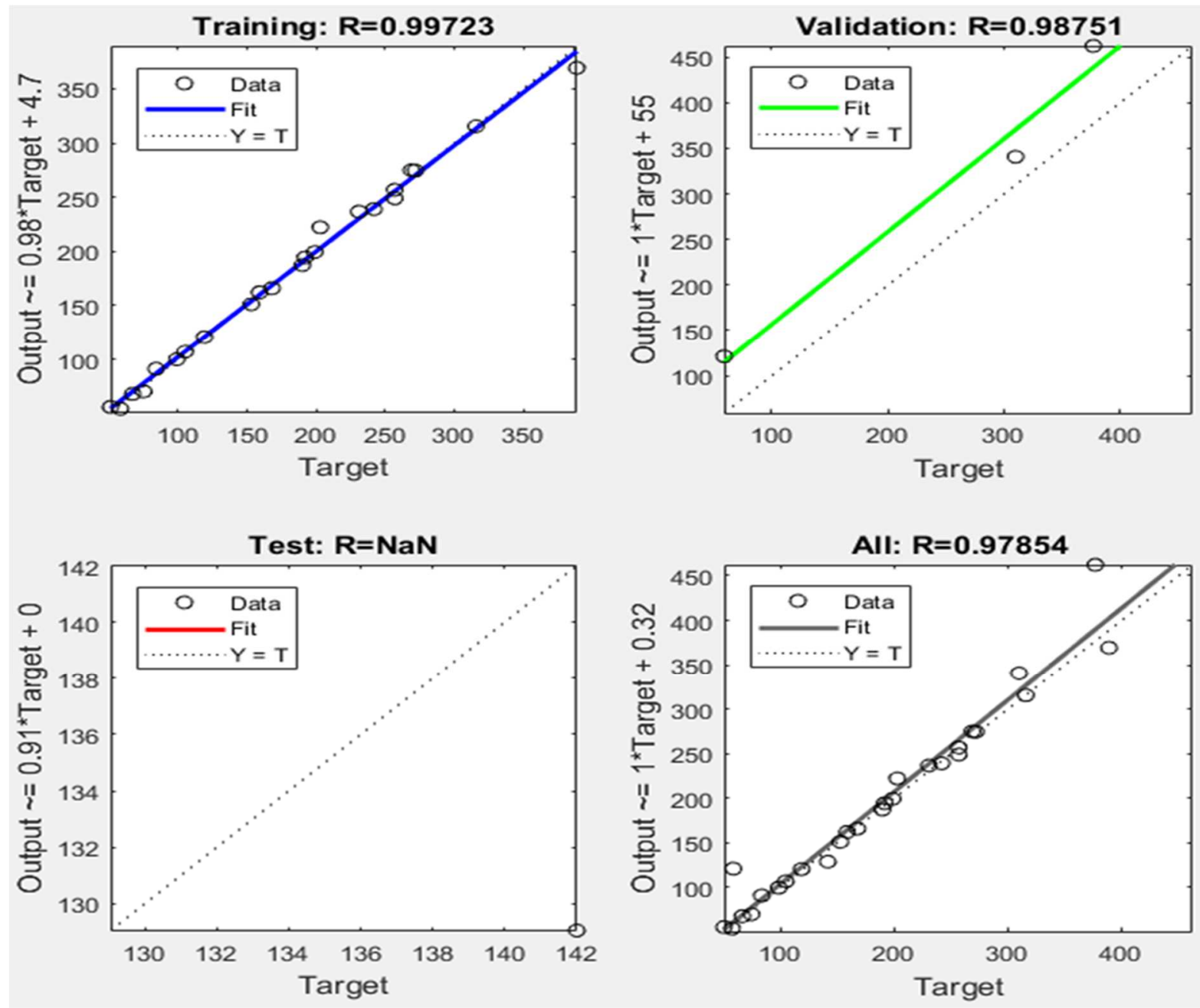

Figure 8: 85\% Training, 10\% Validation, 5\% Testing and 10 Hidden Neurons

\section{$80 \%$ Training, $10 \%$ Validation, $10 \%$ Testing and 10 Hidden Neurons}

Figure 9 shows the regression plot for $80 \%$ training, 10\% validation, 10\% testing and 10 hidden neurons. This gives regression values of 0.99995 for training, 0.99955 for validation, 0.98658 for testing and 0.99553 for all. 

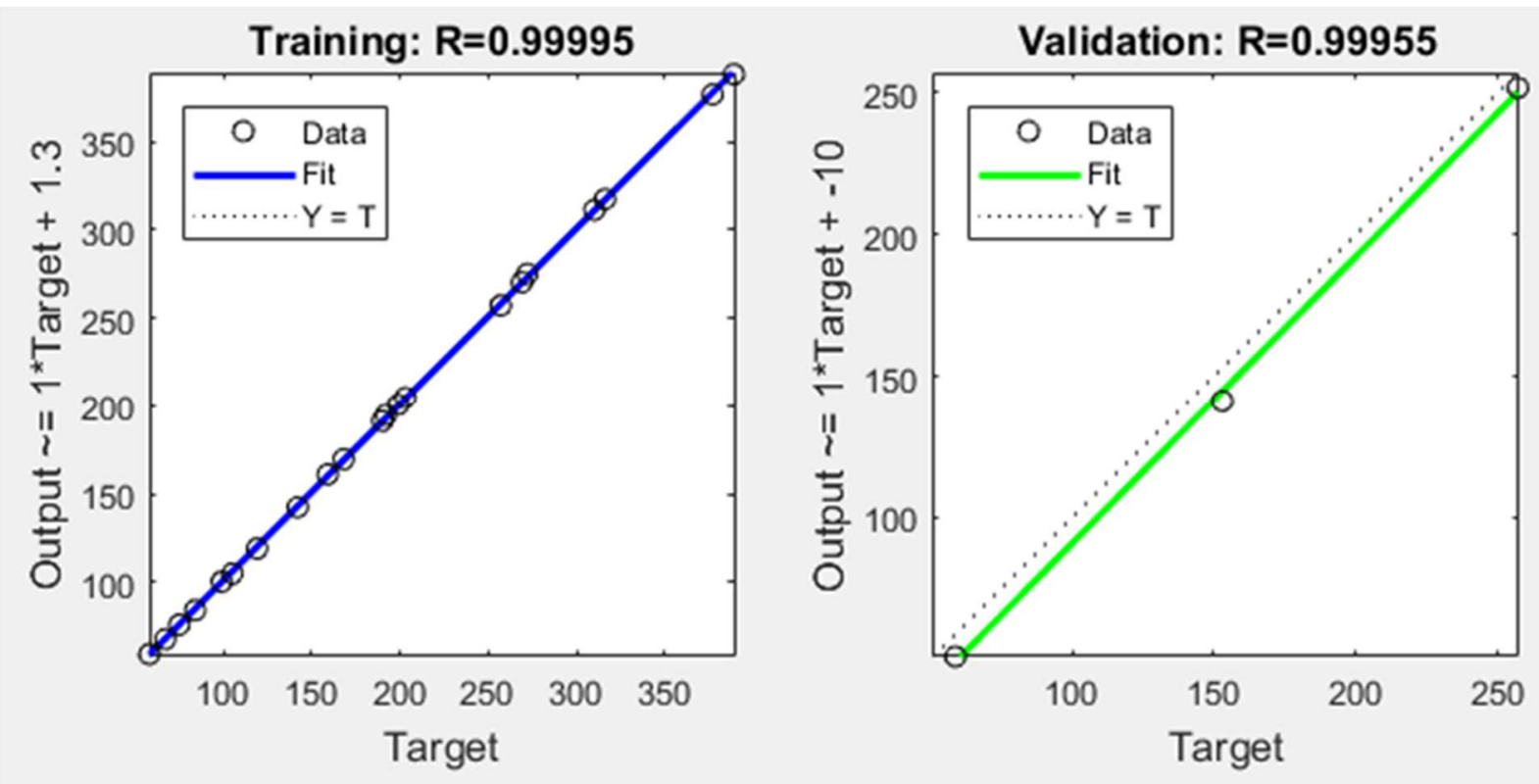

Test: $\mathbf{R}=\mathbf{0 . 9 8 6 5 8}$
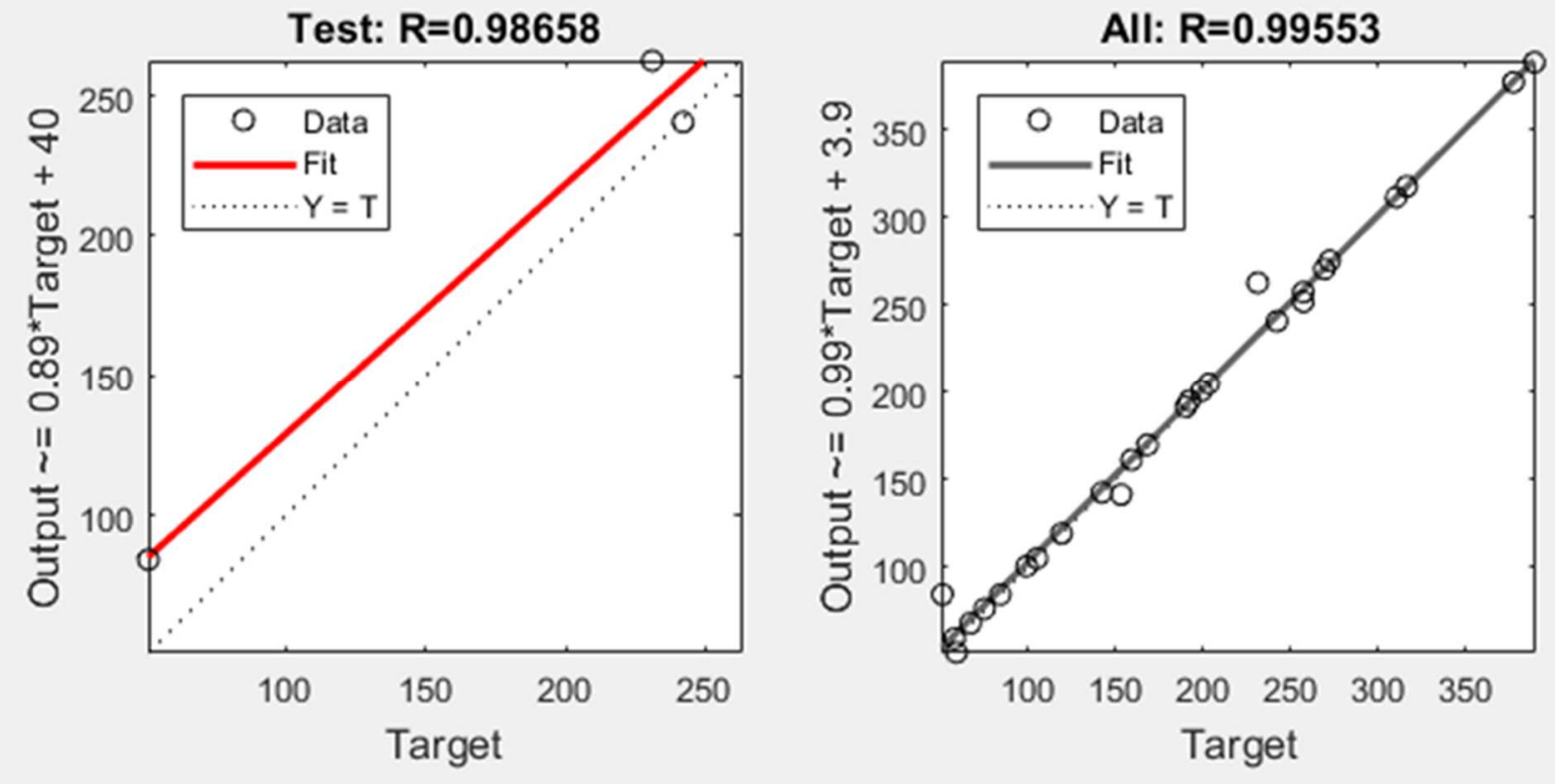

Figure 9: $80 \%$ Training, 10\% Validation, 10\% Testing and 10 Hidden Neurons

\section{5\% Training, 10\% Validation, 15\% Testing and 10 Hidden Neurons}

Figure 10 shows the regression plot for $75 \%$ training, 10\% validation, 15\% testing and 10 hidden neurons. This gives regression values of 1.0 for training, 0.99988 for validation, 0.99277 for testing and 0.99077 for all. 


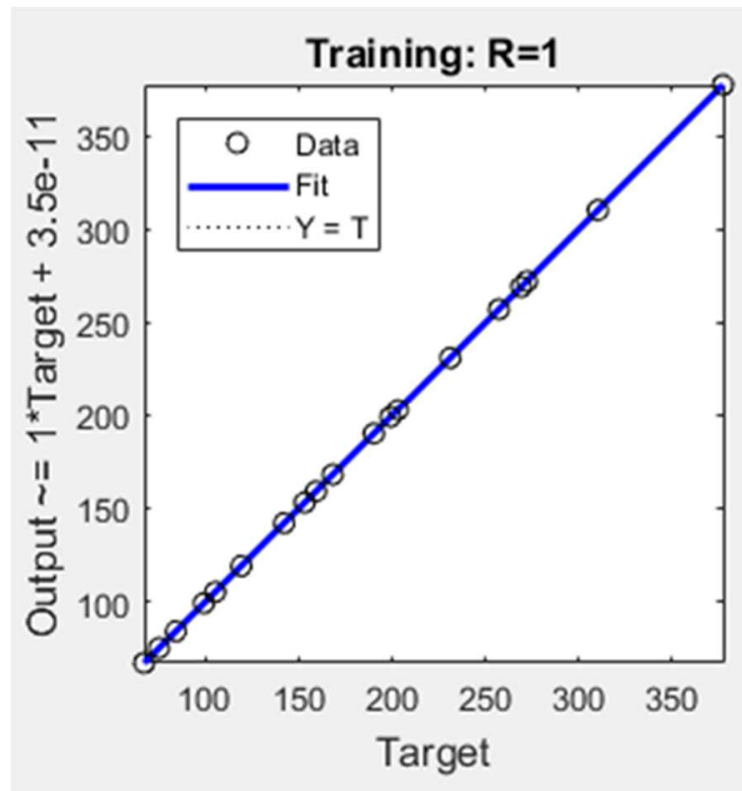

Test: $\mathbf{R}=\mathbf{0 . 9 9 2 7 7}$

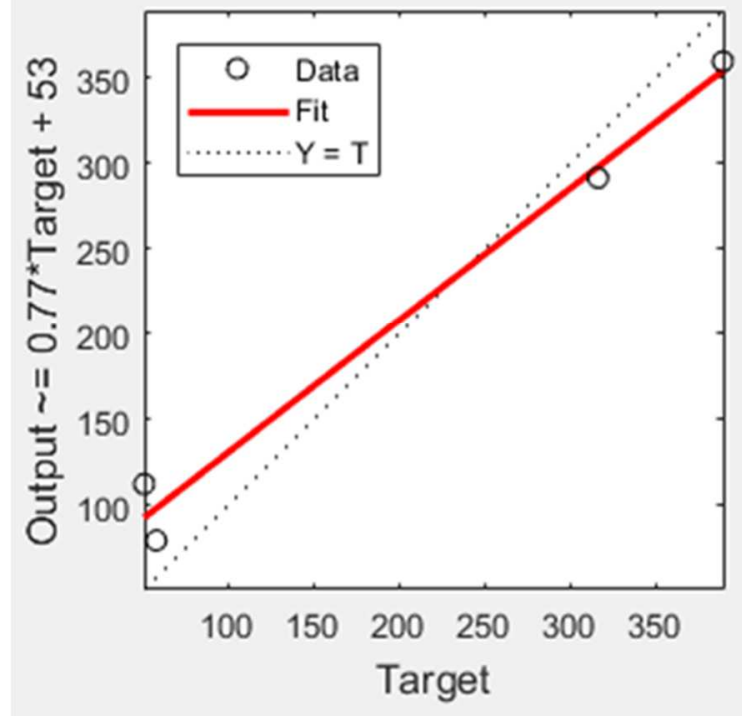

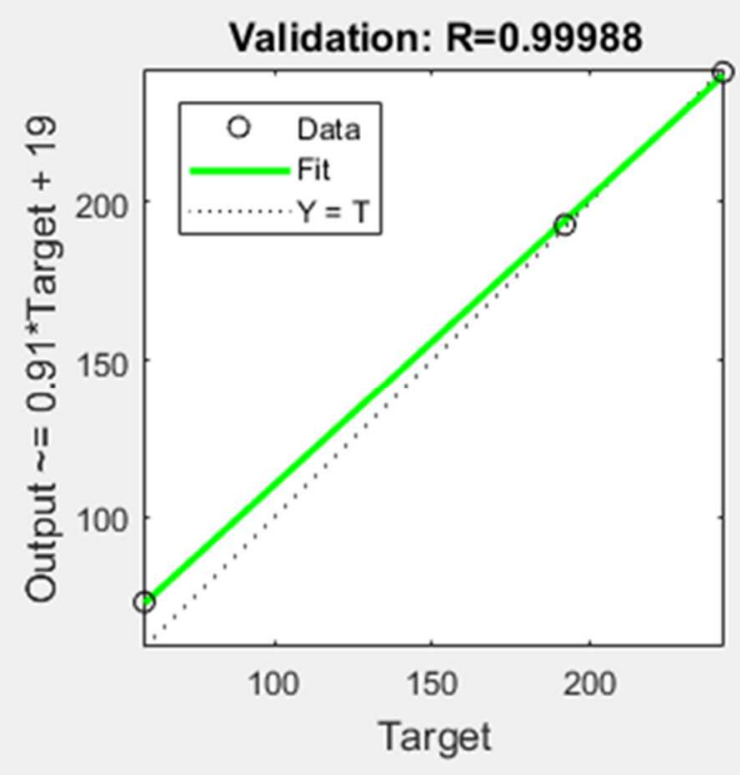

All: $\mathbf{R}=\mathbf{0 . 9 9 0 7 7}$

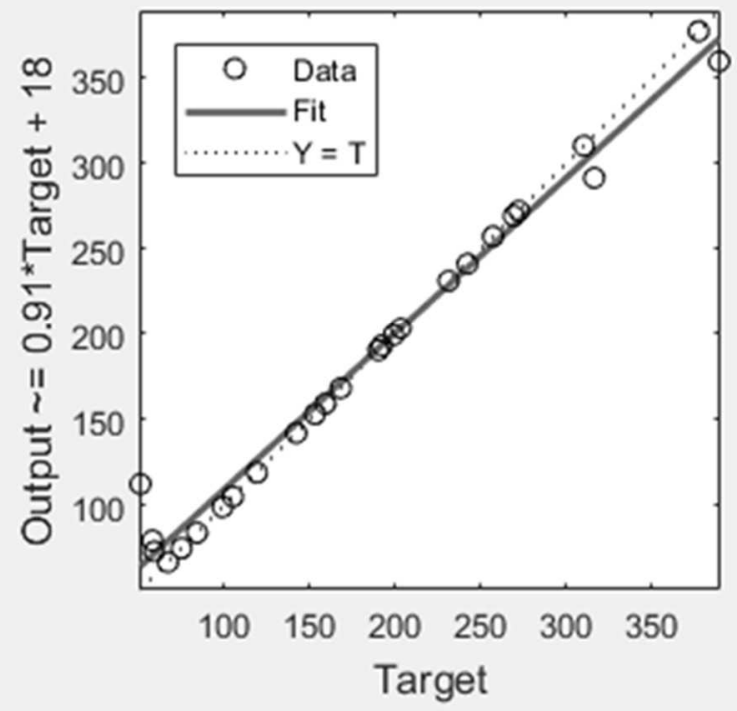

Figure 10: 75\% Training, 10\% Validation, 15\% Testing and 10 Hidden Neurons

70\% Training, 10\% Validation, 20\% Testing and 10 Hidden Neurons

Figure 11 shows the regression plot for $70 \%$ training, 10\% validation, $20 \%$ testing and 10 hidden neurons. This gives regression values of 1.0 for training, 0.9772 for validation, 0.98192 for testing and 0.96205 for all. 

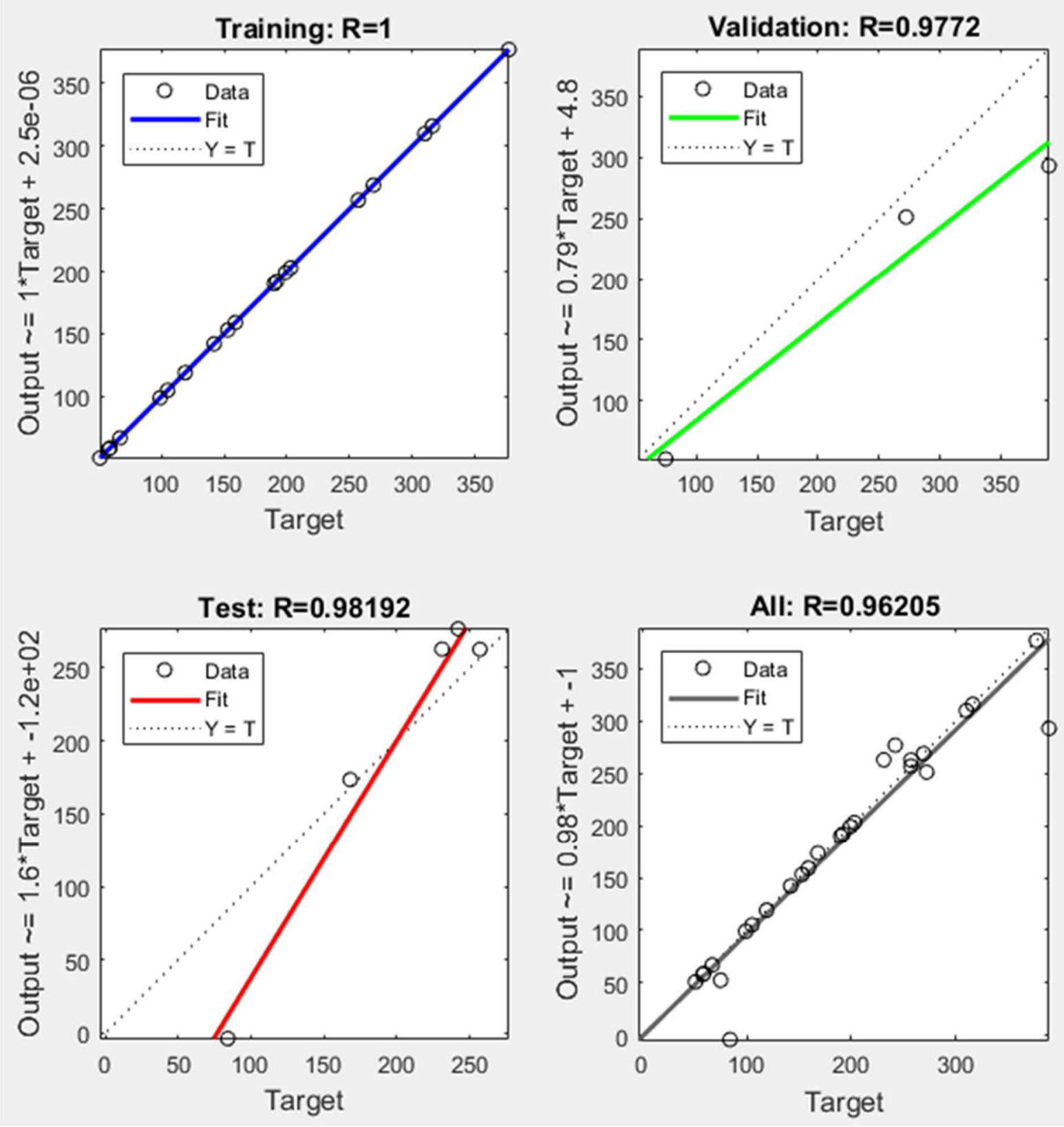

Figure 11: 70\% Training, 10\% Validation, 20\% Testing and 10 Hidden Neurons

\section{5\% Training, 10\% Validation, 25\% Testing and 10 Hidden Neurons}

Figure 12 shows the regression plot for $65 \%$ training, 10\% validation, $25 \%$ testing and 10 hidden neurons. This gives regression values of 0.9916 for training, 0.97457 for validation, 0.99205 for testing and 0.99044 for all. 

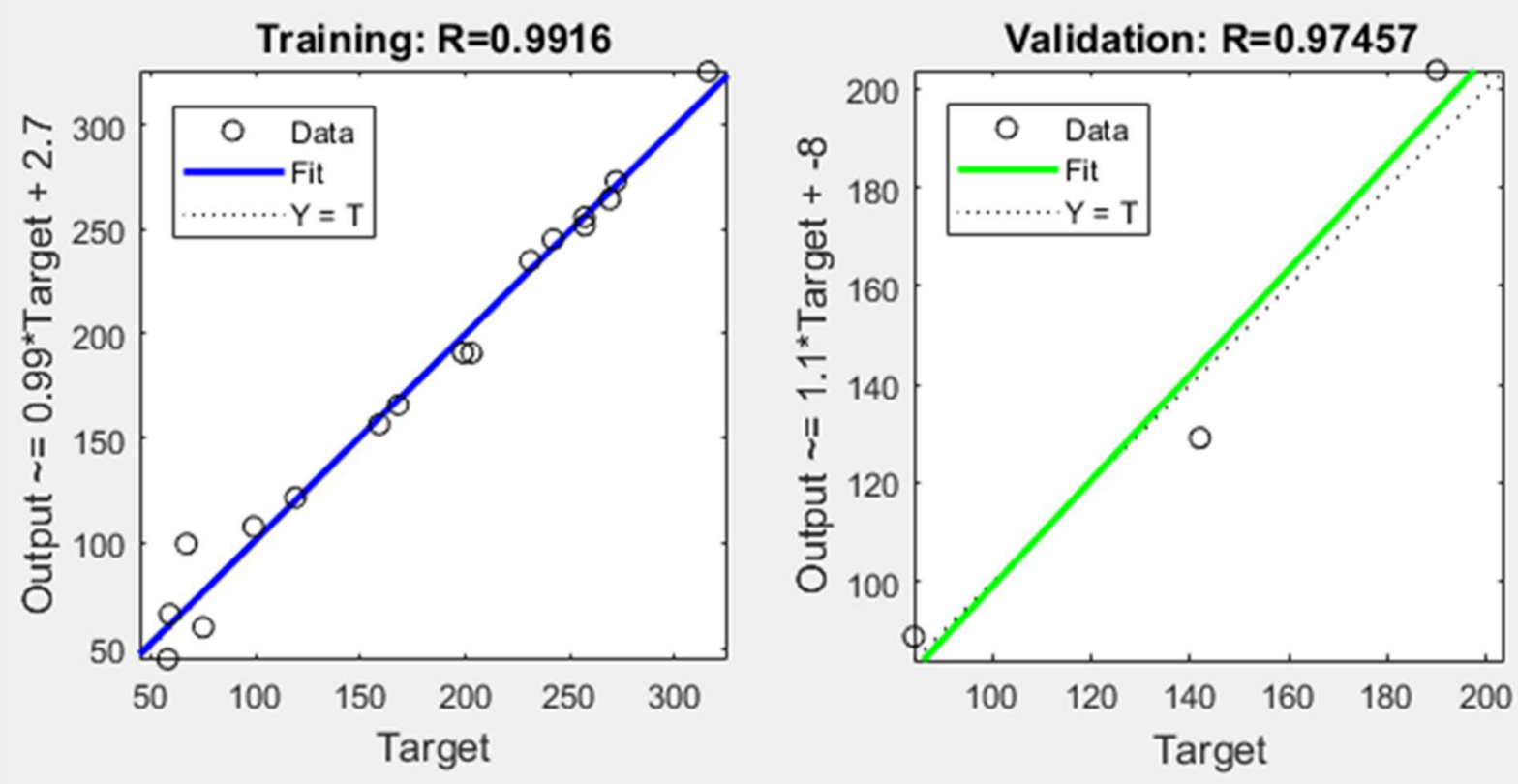

Test: $\mathbf{R}=\mathbf{0 . 9 9 2 0 5}$
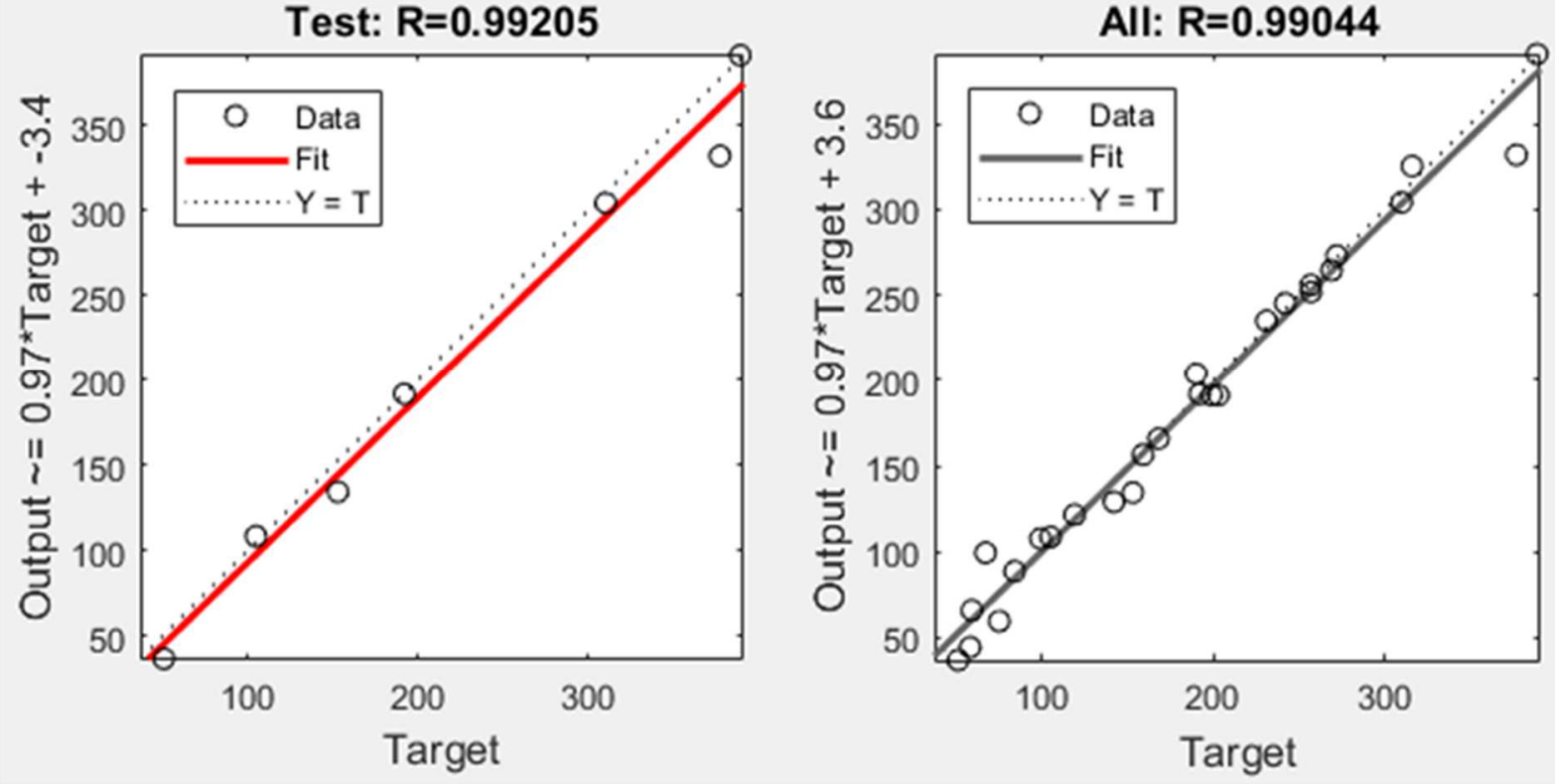

Figure 12: 65\% Training, 10\% Validation, 25\% Testing and 10 Hidden Neurons $60 \%$ Training, $10 \%$ Validation, 30\% Testing and 10 Hidden Neurons

Figure 13 shows the regression plot for $60 \%$ training, $10 \%$ validation, $30 \%$ testing and 10 hidden neurons. This gives regression values of 0.99181 for training, 0.995470 for validation, 0.9752 for testing and 0.98086 for all. 

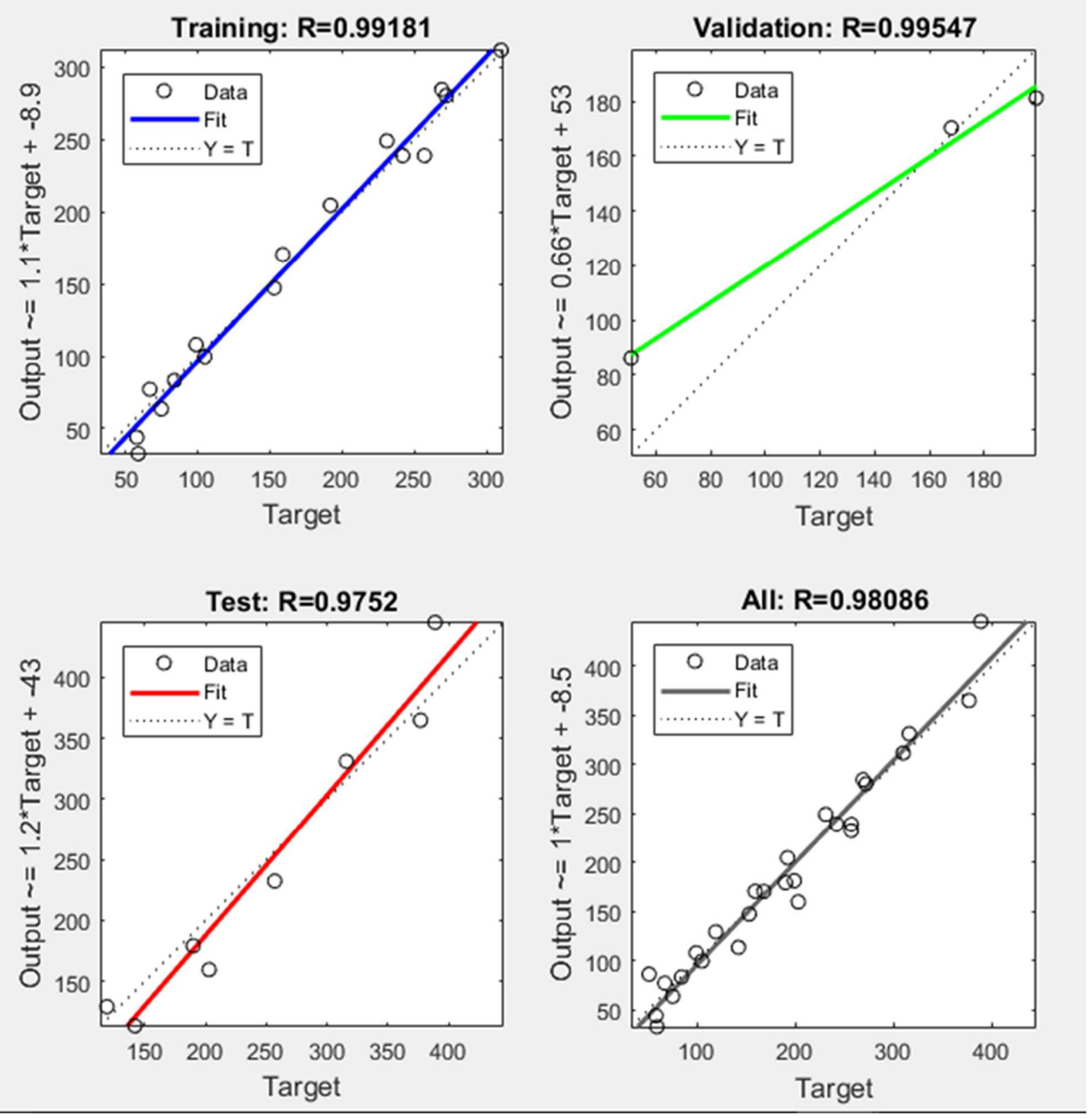

Figure 13: $60 \%$ Training, 10\% Validation, 30\% Testing and 10 Hidden Neurons

\section{5\% Training, $10 \%$ Validation, 35\% Testing and 10 Hidden Neurons}

Figure 14 shows the regression plot for 55\% training, 10\% validation, 35\% testing and 10 hidden neurons. This gives regression values of 0.99538 for training, 0.99999 for validation, 0.99442 for testing and 0.99037 for all. 

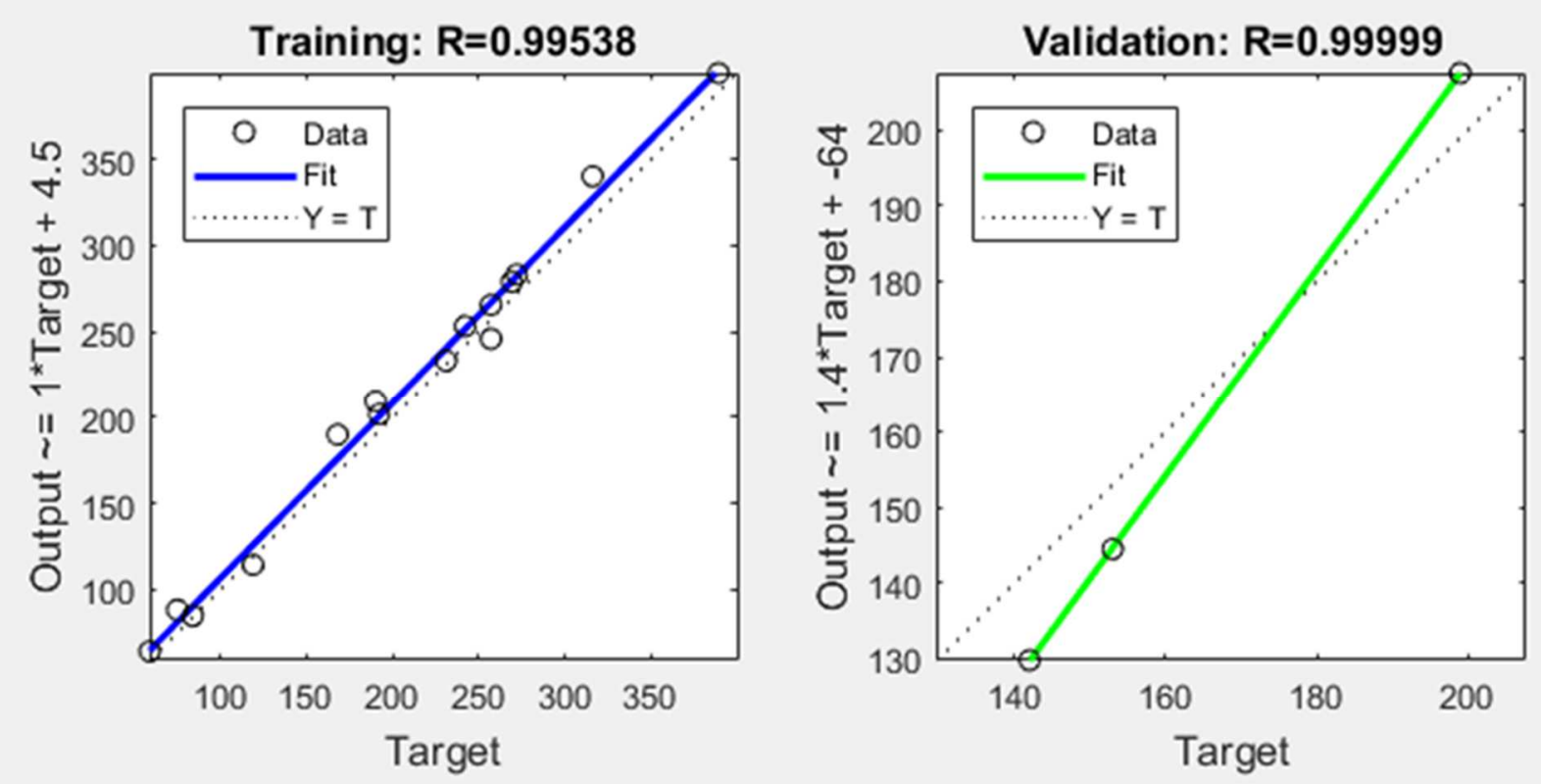

Test: $\mathbf{R}=\mathbf{0 . 9 9 4 4 2}$
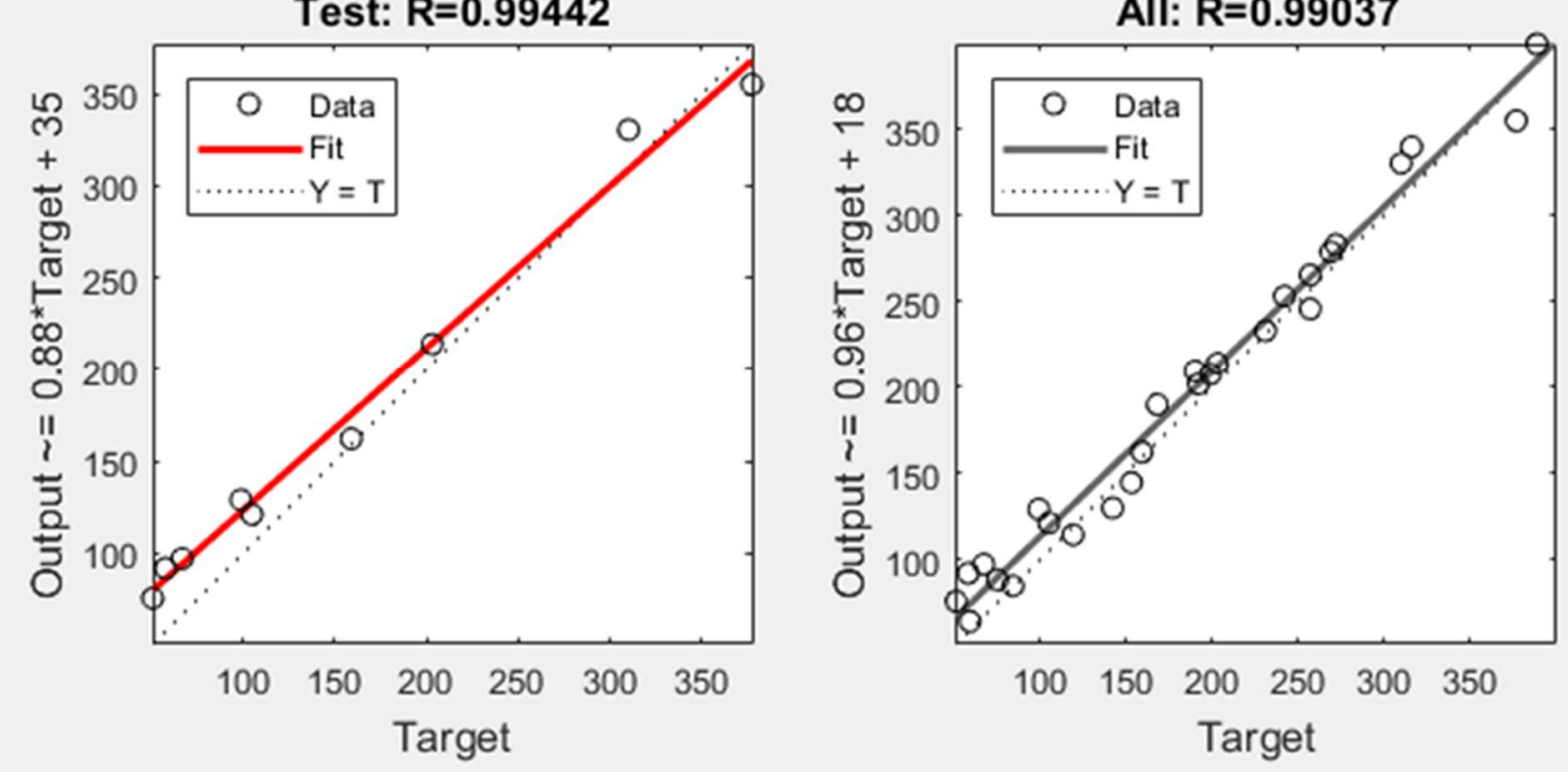

Figure 14: 55\% Training, 10\% Validation, 35\% Testing and 10 Hidden Neurons

$80 \%$ Training, $15 \%$ Validation, 5\% Testing and 10 Hidden Neurons 

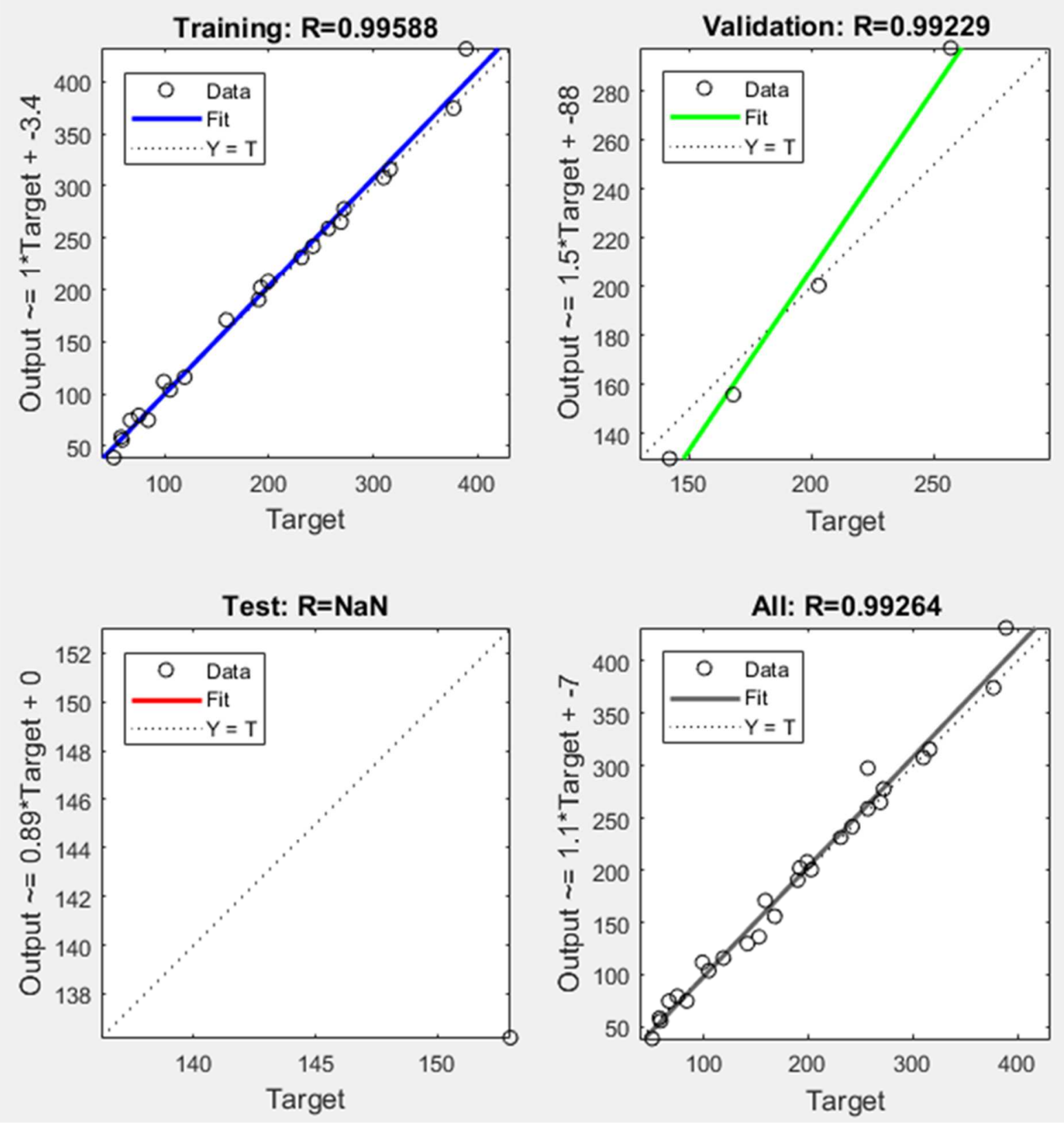

Figure 15: 80\% Training, 15\% Validation, 5\% Testing and 10 Hidden Neurons

\section{5\% Training, $15 \%$ Validation, $10 \%$ Testing and 10 Hidden Neurons}

Figure 16 shows the regression plot for $75 \%$ training, $15 \%$ validation, $10 \%$ testing and 10 hidden neurons. This gives regression values of 1.0 for training, 0.98076 for validation, 0.99999 for testing and 0.99635 for all. 

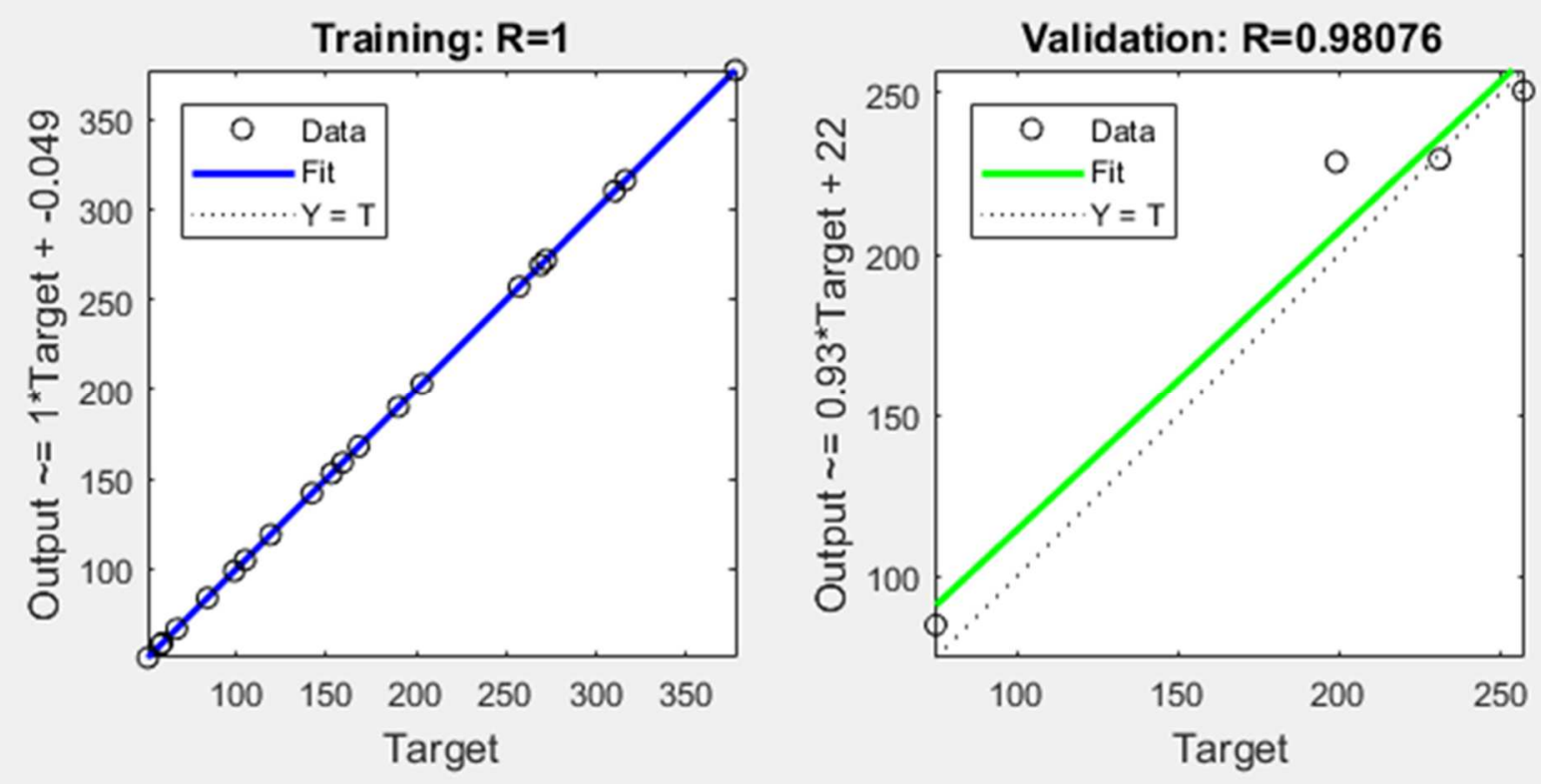

Test: $\mathbf{R}=\mathbf{0 . 9 9 9 9 9}$
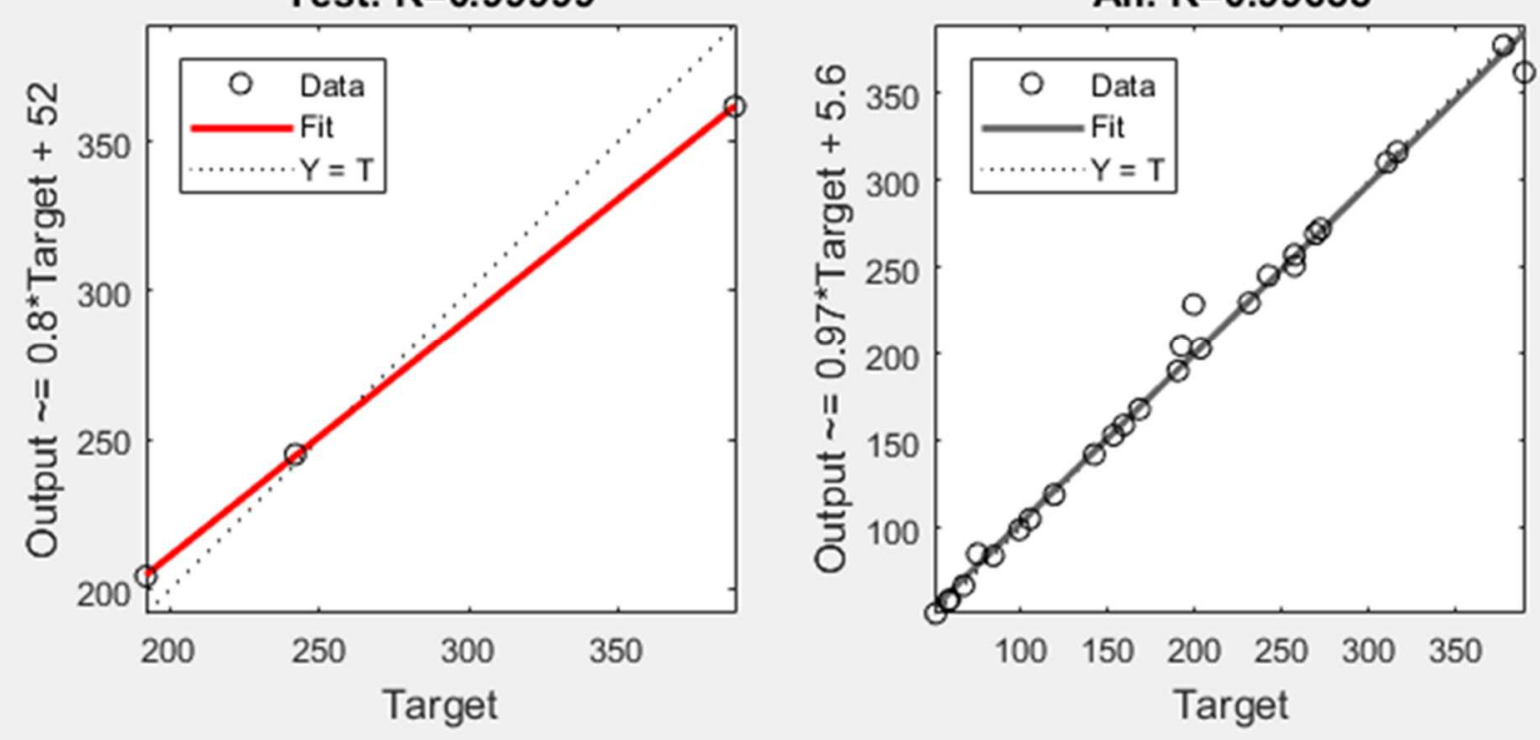

Figure 16: 75\% Training, 15\% Validation, $10 \%$ Testing and 10 Hidden Neurons

70\% Training, $15 \%$ Validation, $15 \%$ Testing and 10 Hidden Neurons

Figure 17 shows the regression plot for $70 \%$ training, $15 \%$ validation, $15 \%$ testing and 10 hidden neurons. This gives regression values of 1.0 for training, 0.95958 for validation, 0.99948 for testing and 0.993670 for all. 

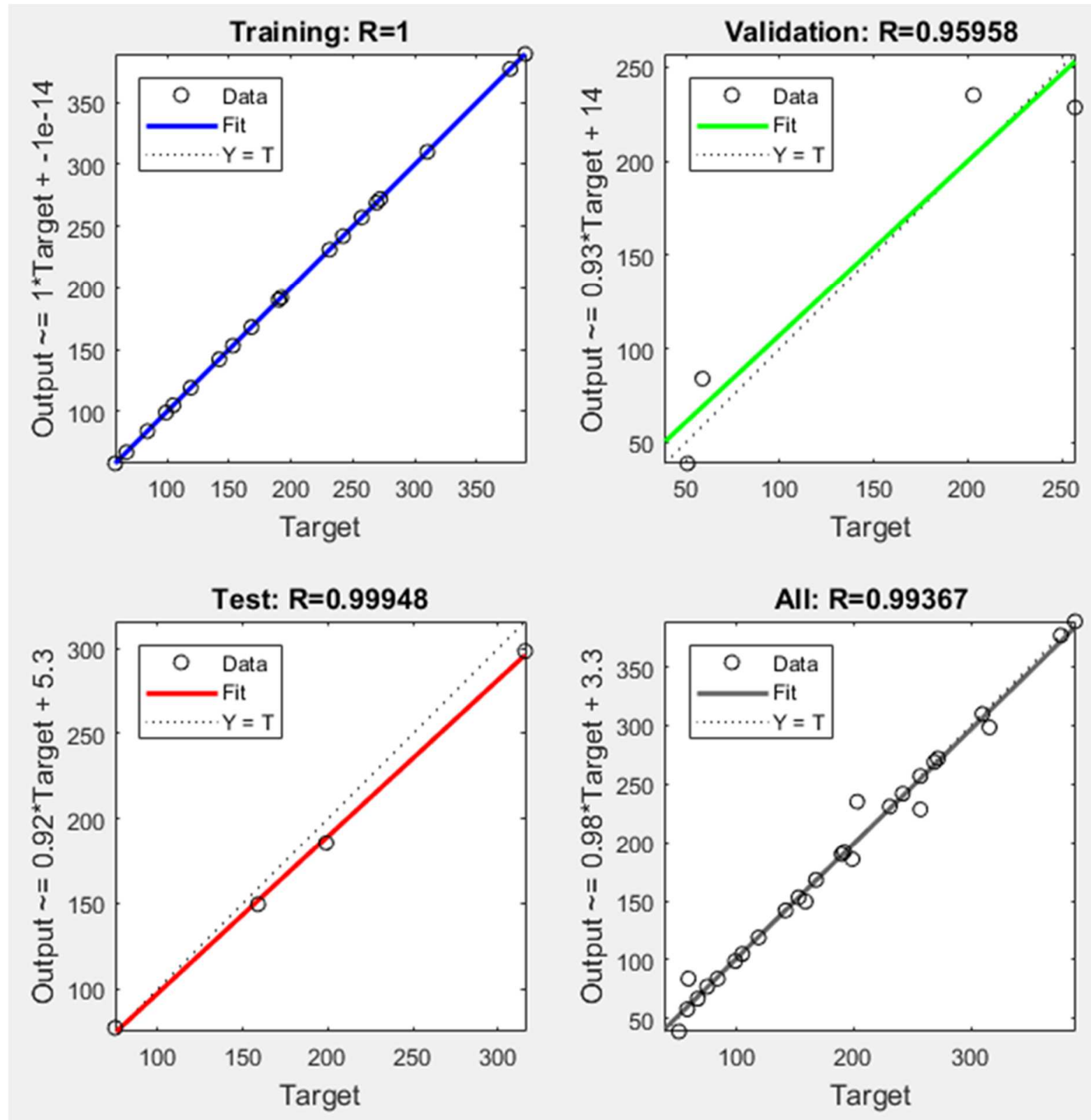

Figure 17: 70\% Training, 15\% Validation, 15\% Testing and 10 Hidden Neurons

\section{5\% Training, 15\% Validation, 20\% Testing and 10 Hidden Neurons}

Figure 18 shows the regression plot for $65 \%$ training, $15 \%$ validation, $20 \%$ testing and 10 hidden neurons. This gives regression values of 1.0 for training, 0.98873 for validation, 0.98463 for testing and 0.98317 for all. 

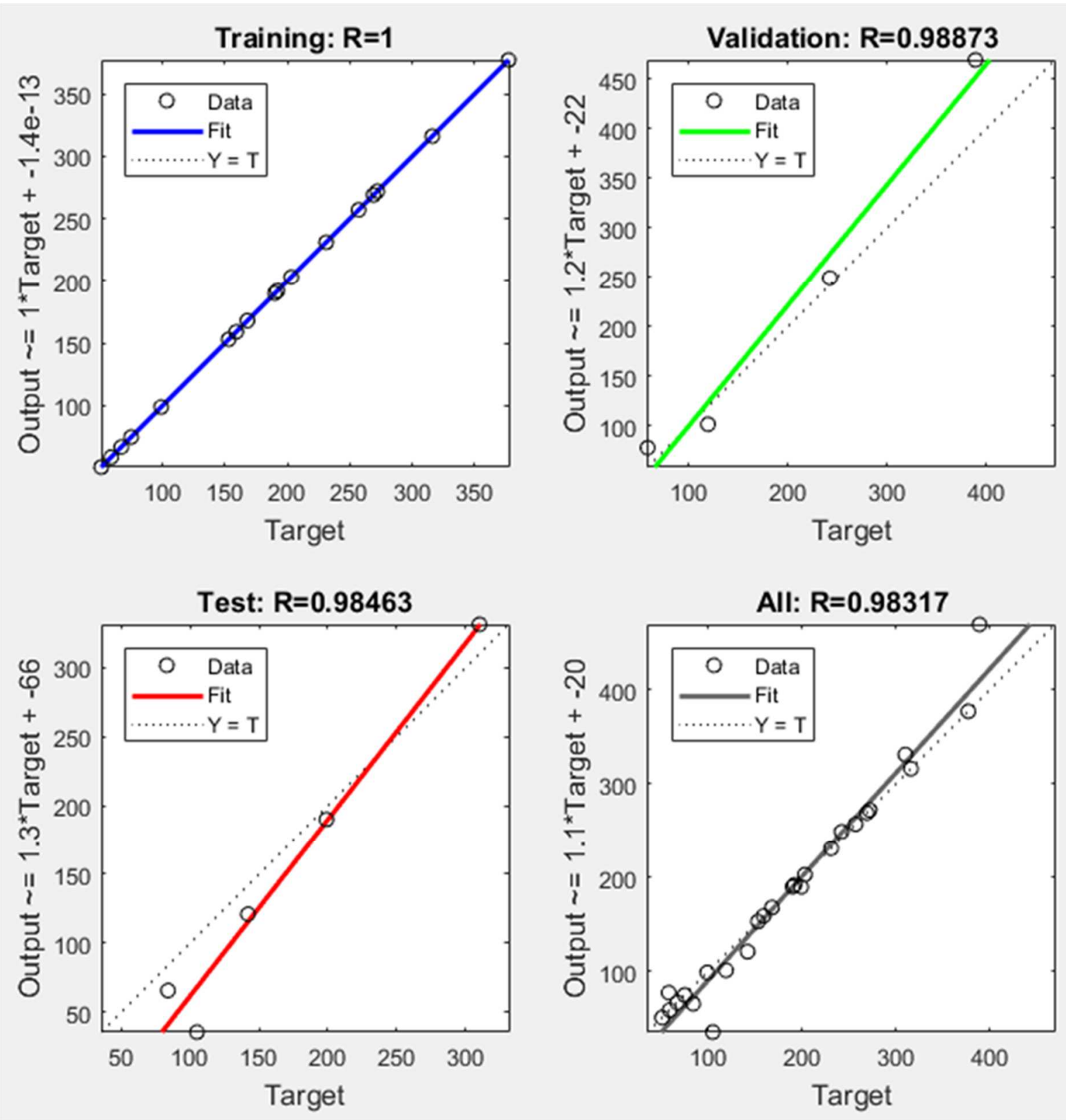

Figure 18: 65\% Training, 15\% Validation, 20\% Testing and 10 Hidden Neurons

$60 \%$ Training, $15 \%$ Validation, 25\% Testing and 10 Hidden Neurons

Figure 19 shows the regression plot for $60 \%$ training, $15 \%$ validation, $25 \%$ testing and 10 hidden neurons. This gives regression values of 0.96724 for training, 0.98844 for validation, 0.99561 for testing and 0.97329 for all. 

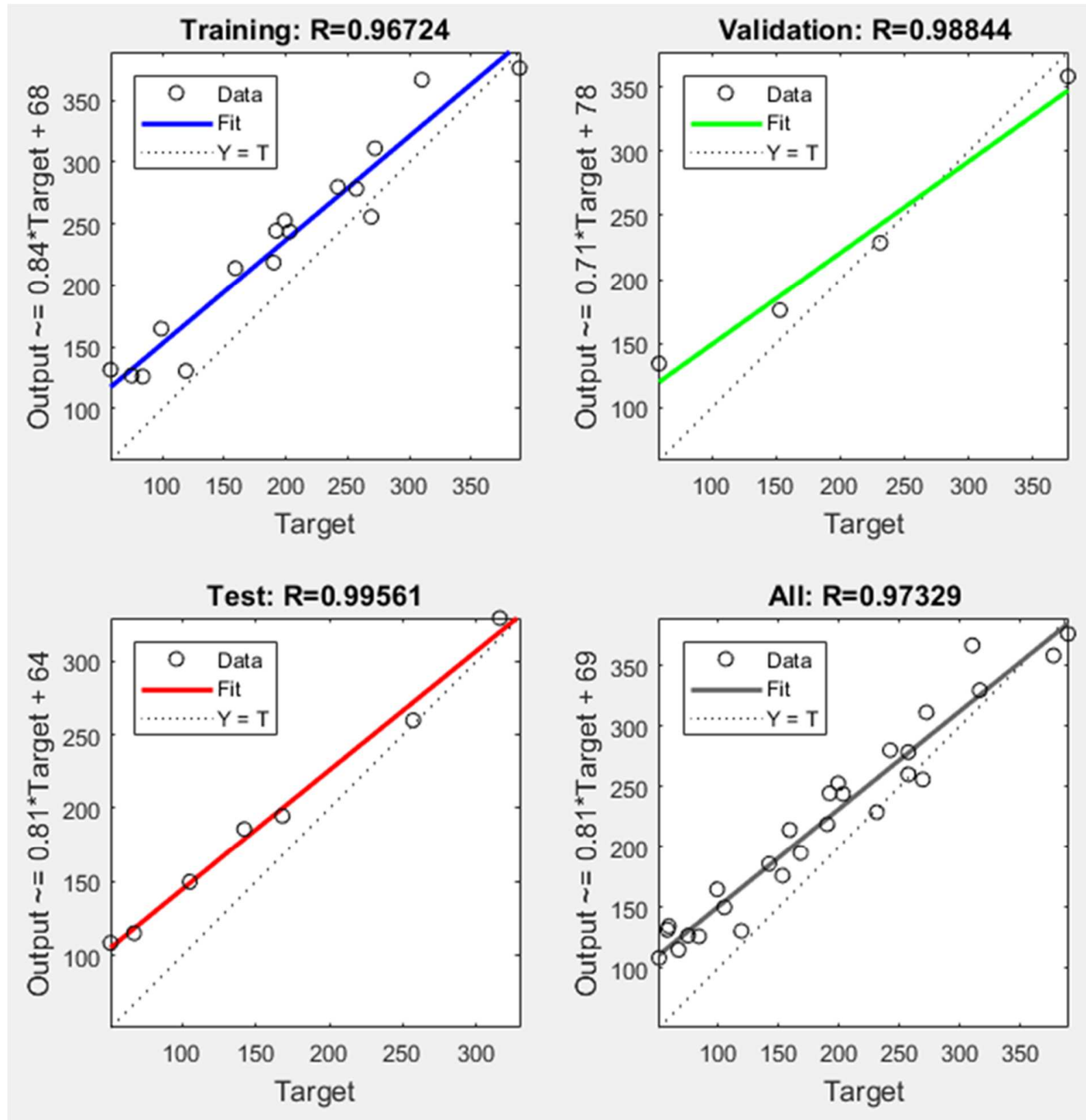

Figure 19: 60\% Training, 15\% Validation, 25\% Testing and 10 Hidden Neurons

$55 \%$ Training, $15 \%$ Validation, $30 \%$ Testing and 10 Hidden Neurons

Figure 20 shows the regression plot for 55\% training, 15\% validation, 30\% testing and 10 hidden neurons. This gives regression values of 0.99583 for training, 0.95891 for validation, 0.96685 for testing and 0.97082 for all. 

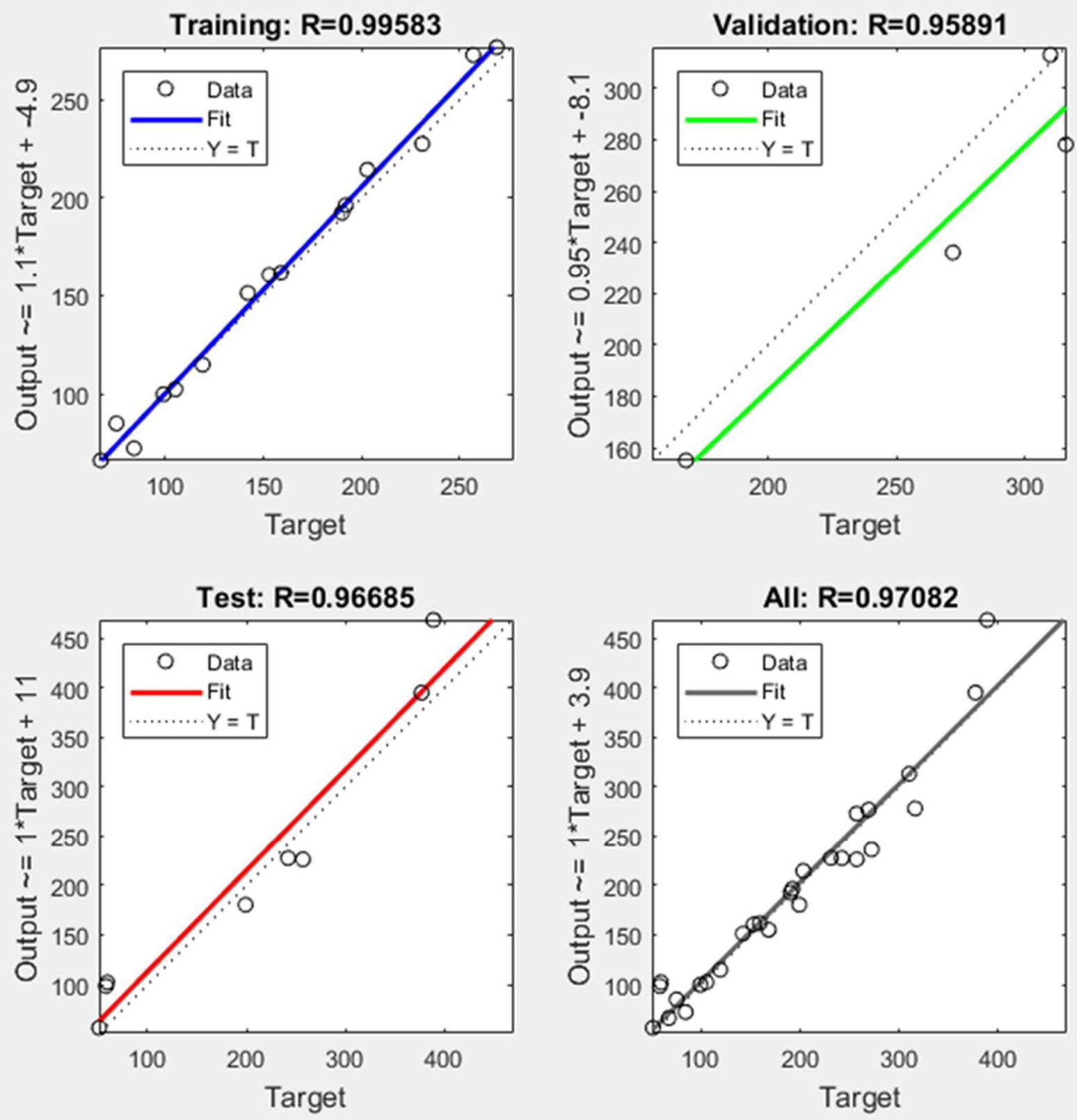

Figure 20: 55\% Training, 15\% Validation, 30\% Testing and 10 Hidden Neurons

50\% Training, 15\% Validation, 35\% Testing and 10 Hidden Neurons

Figure 21 shows the regression plot for 50\% training, 15\% validation, 35\% testing and 10 hidden neurons. This gives regression values of 0.99651 for training, 0.93055 for validation, 0.93578 for testing and 0.95265 for all. 

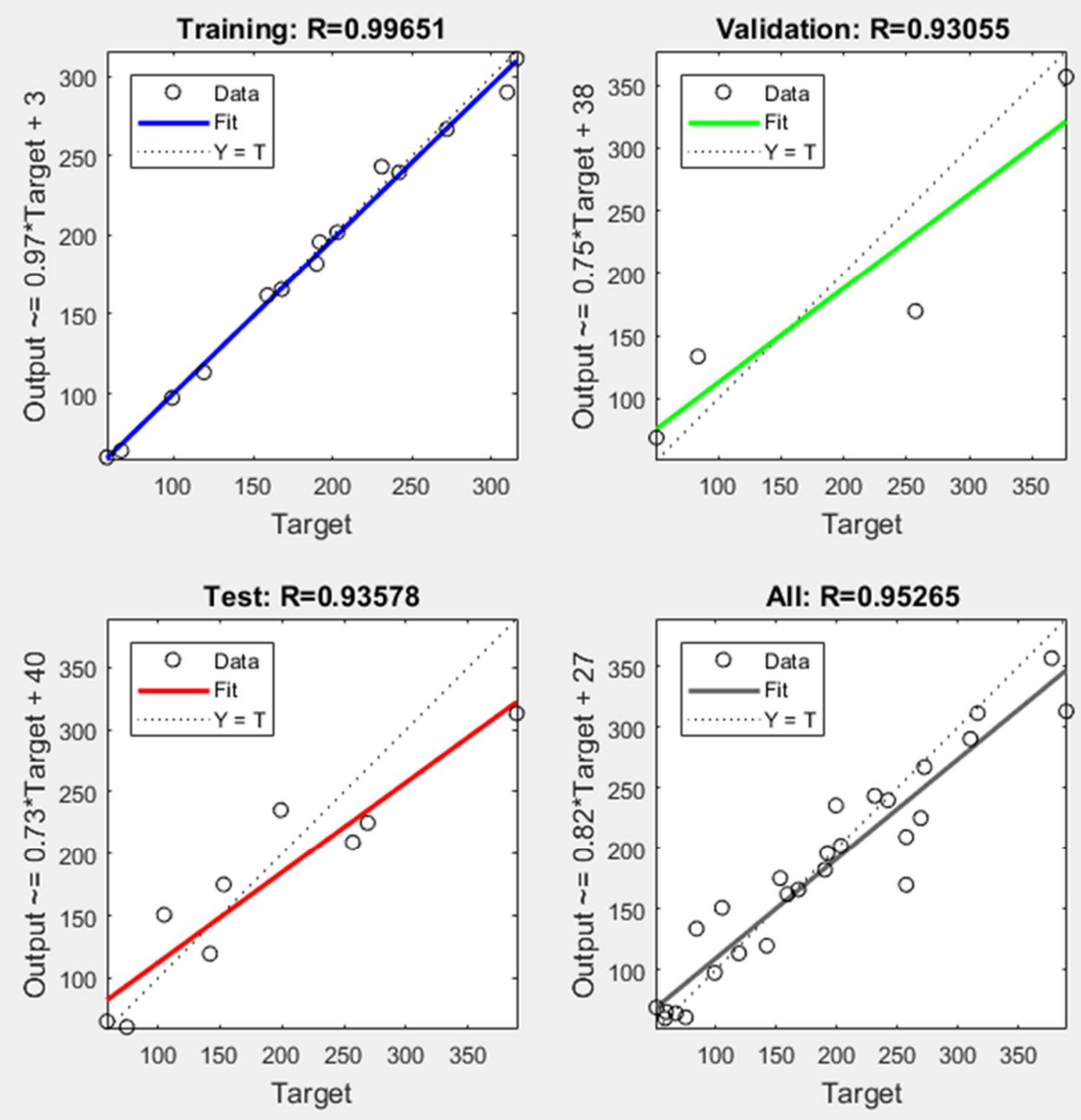

Figure 21: 50\% Training, 15\% Validation, 35\% Testing and 10 Hidden Neurons

75\% Training, 20\% Validation, 5\% Testing and 10 Hidden Neurons 

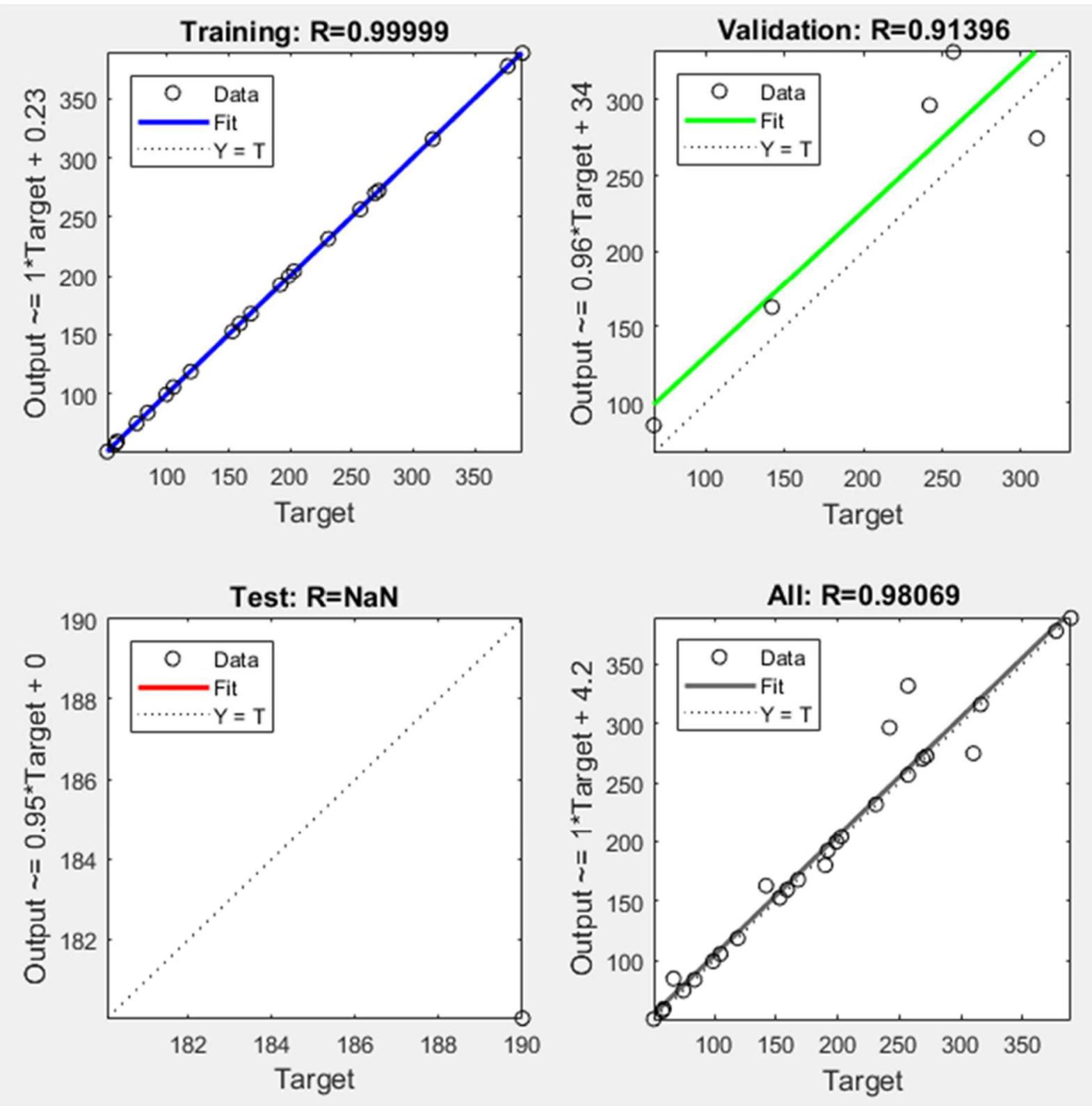

Figure 22: 75\% Training, 20\% Validation, 5\% Testing and 10 Hidden Neurons

70\% Training, 20\% Validation, $10 \%$ Testing and 10 Hidden Neurons

Figure 23 shows the regression plot for $70 \%$ training, $20 \%$ validation, $10 \%$ testing and 10 hidden neurons. This gives regression values of 1.0 for training, 0.99527 for validation, 1.0 for testing and 0.99667 for all. 

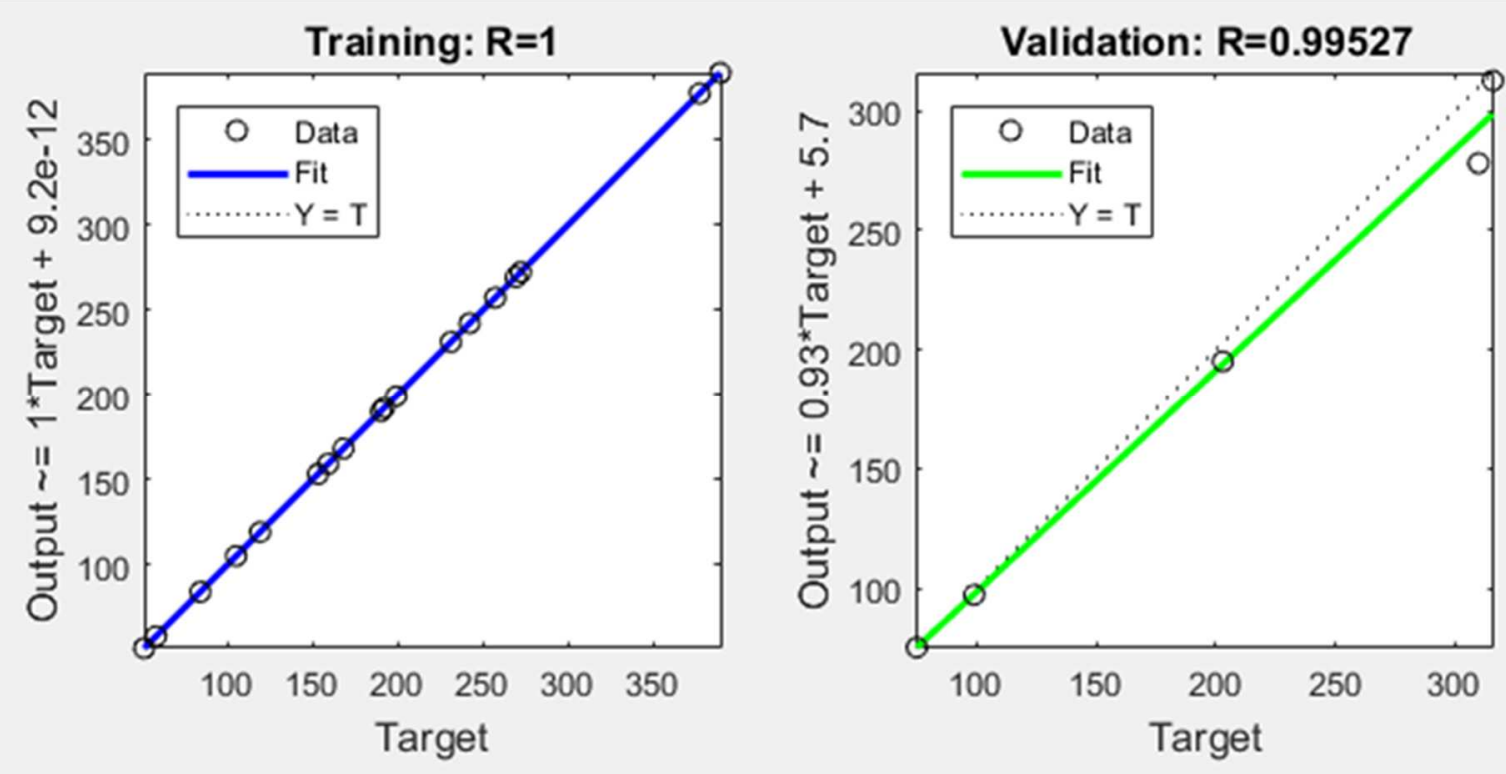

\section{Test: $\mathbf{R = 1}$}
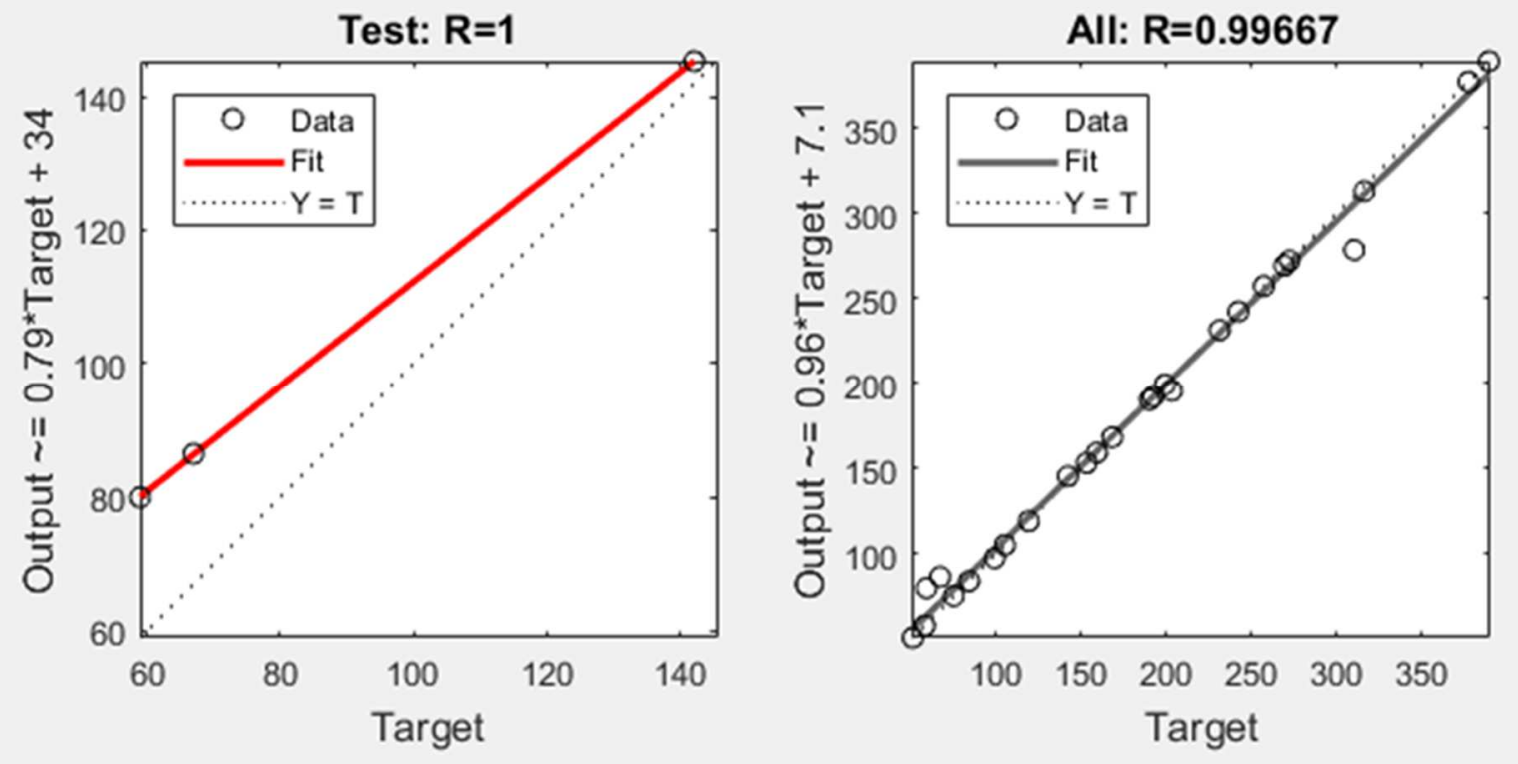

Figure 23: 70\% Training, 20\% Validation, $10 \%$ Testing and 10 Hidden Neurons

\section{5\% Training, 20\% Validation, 15\% Testing and 10 Hidden Neurons}

Figure 24 shows the regression plot for $65 \%$ training, 20\% validation, $15 \%$ testing and 10 hidden neurons. This gives regression values of 1.0 for training, 0.9648 for validation, 0.99826 for testing and 0.99332 for all. 

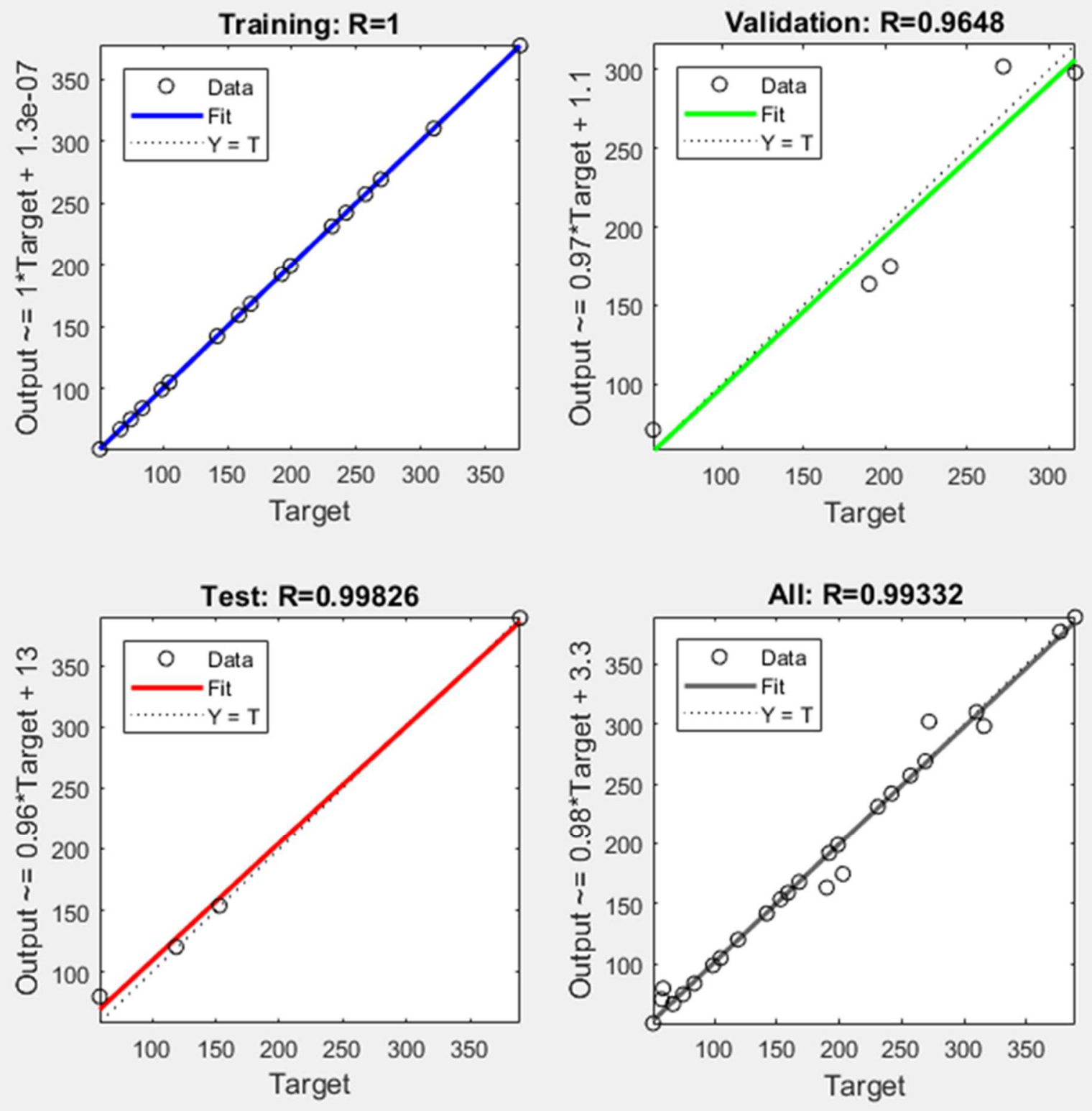

Figure 24: 65\% Training, 20\% Validation, 15\% Testing and 10 Hidden Neurons

$60 \%$ Training, 20\% Validation, 20\% Testing and 10 Hidden Neurons

Figure 25 shows the regression plot for $60 \%$ training, 20\% validation, $20 \%$ testing and 10 hidden neurons. This gives regression values of 0.998260 for training, 0.98592 for validation, 0.99296 for testing and 0.98068 for all. 

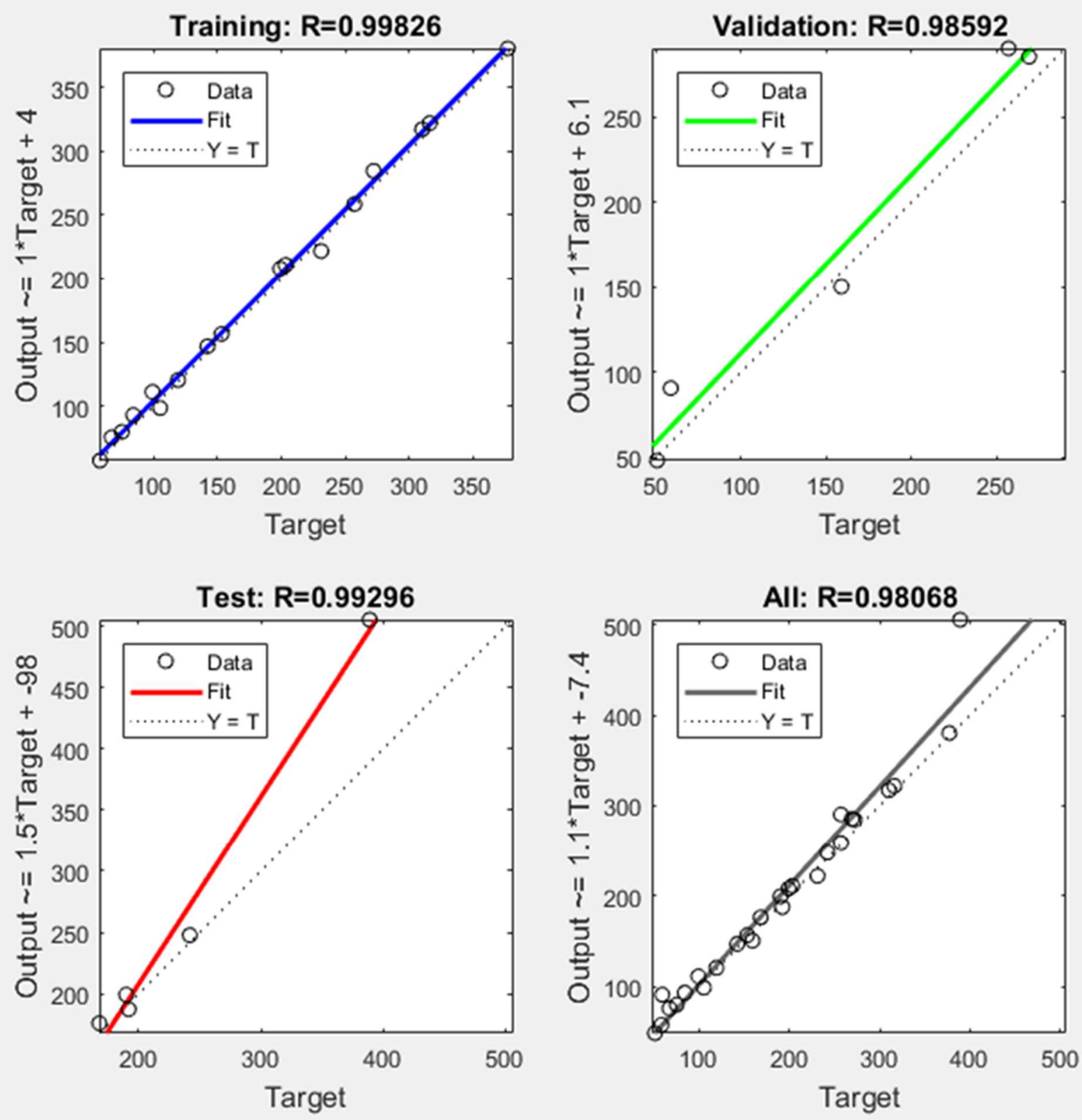

Figure 25: 60\% Training, 20\% Validation, 20\% Testing and 10 Hidden Neurons

\section{$55 \%$ Training, 20\% Validation, 25\% Testing and 10 Hidden Neurons}

Figure 26 shows the regression plot for 55\% training, 20\% validation, 25\% testing and 10 hidden neurons. This gives regression values of 0.99955 for training, 0.98771 for validation, 0.99047 for testing and 0.99097 for all. 

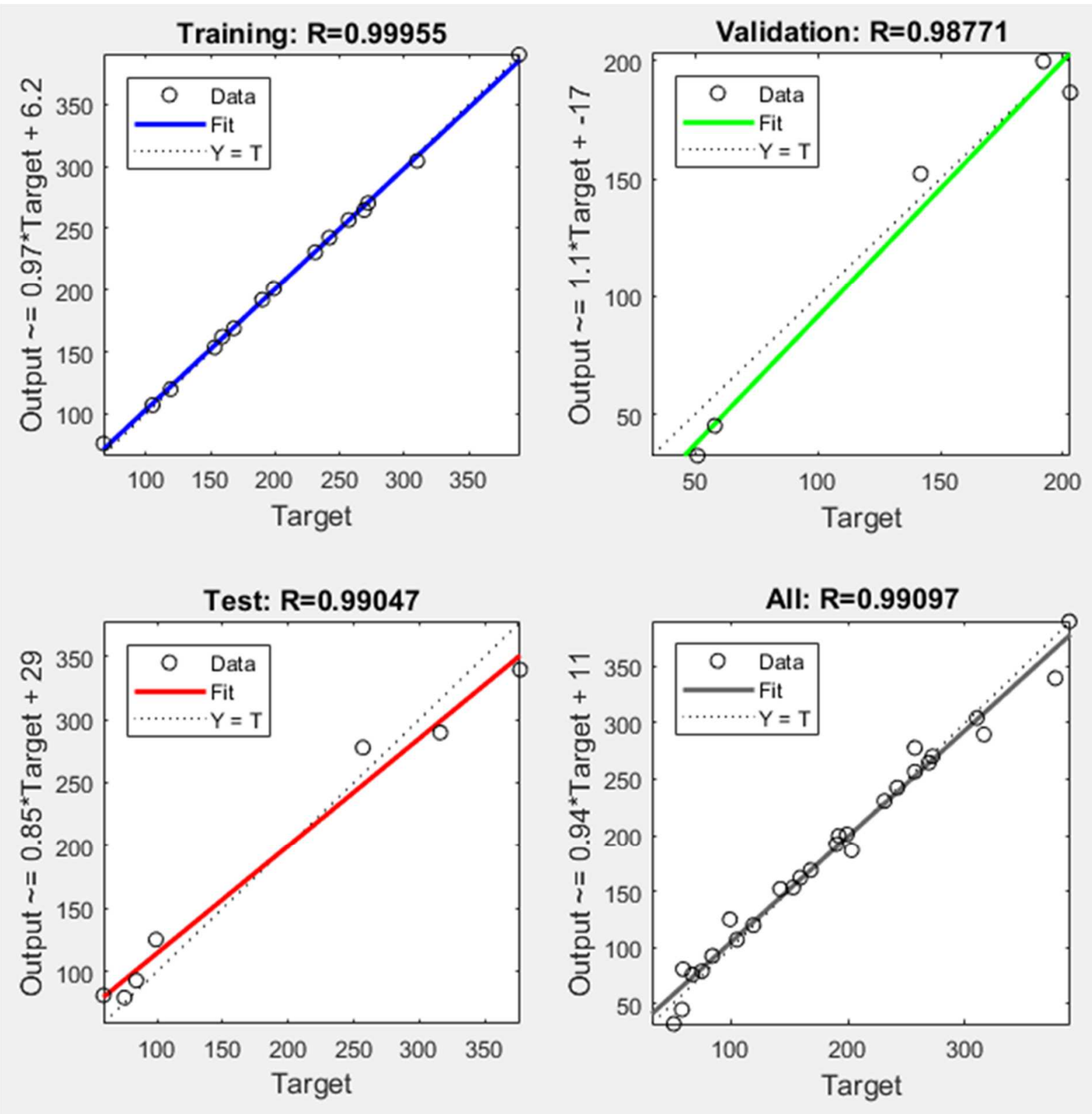

Figure 26: 55\% Training, 20\% Validation, 25\% Testing and 10 Hidden Neurons

$50 \%$ Training, 20\% Validation, $30 \%$ Testing and 10 Hidden Neurons

Figure 27 shows the regression plot for 50\% training, 20\% validation, 30\% testing and 10 hidden neurons. This gives regression values of 1.0 for training, 0.94972 for validation, 0.99261 for testing and 0.97565 for all. 

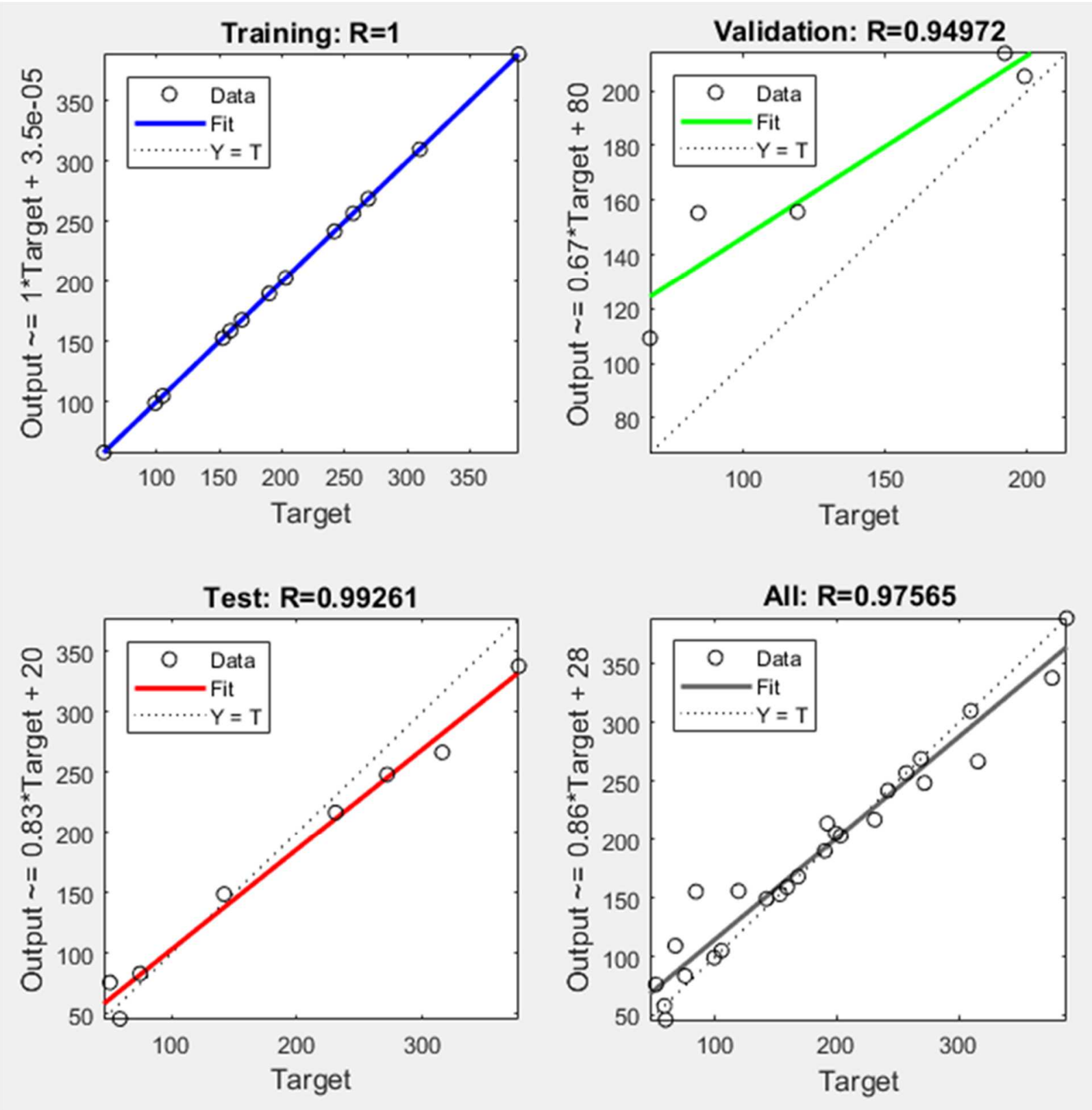

Figure 27: 50\% Training, 20\% Validation, 30\% Testing and 10 Hidden Neurons

\section{5\% Training, 20\% Validation, 35\% Testing and 10 Hidden Neurons}

Figure 28 shows the regression plot for $45 \%$ training, 20\% validation, 35\% testing and 10 hidden neurons. This gives regression values of 1.0 for training, 0.95196 for validation, 0.90767 for testing and 0.96244 for all. 

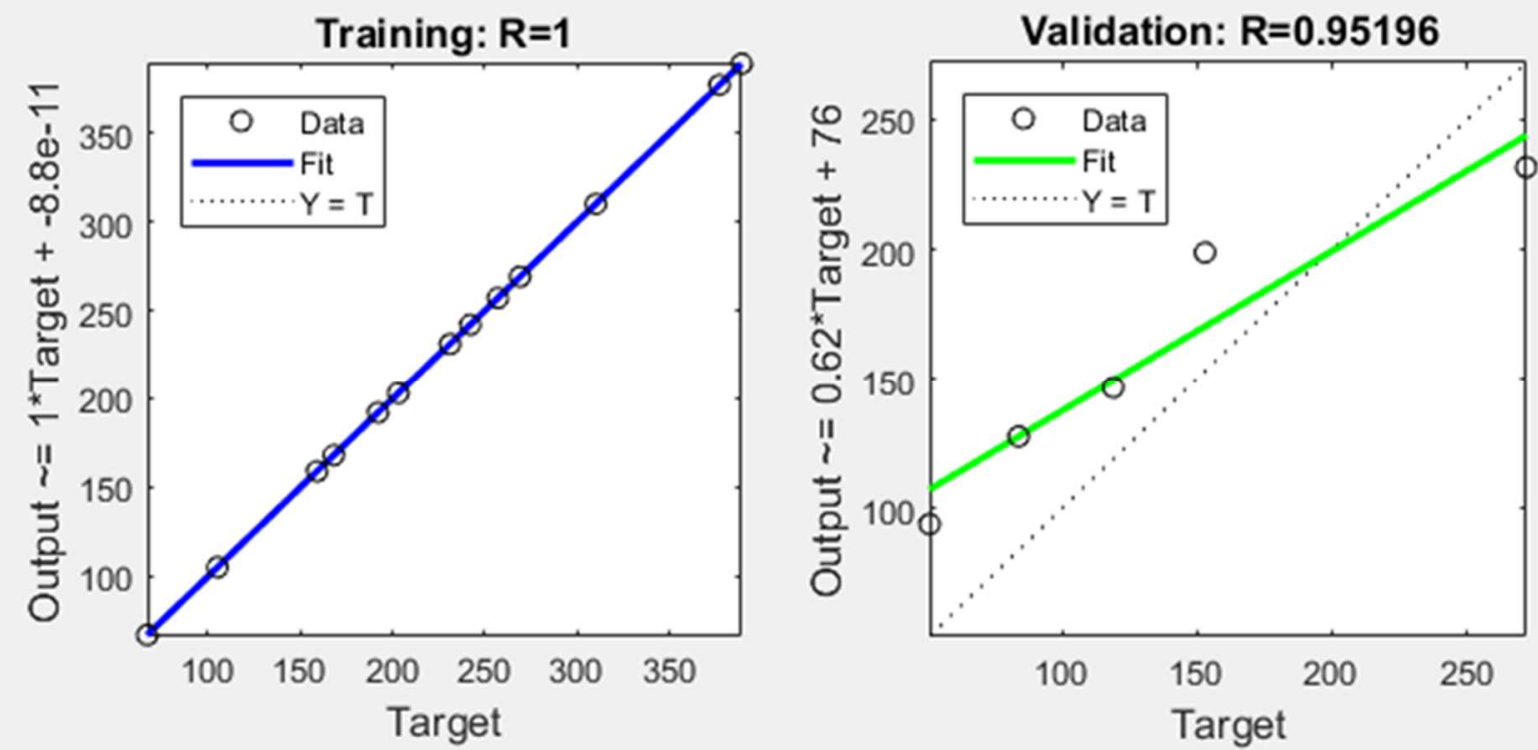

Test: $\mathbf{R}=\mathbf{0 . 9 0 7 6 7}$
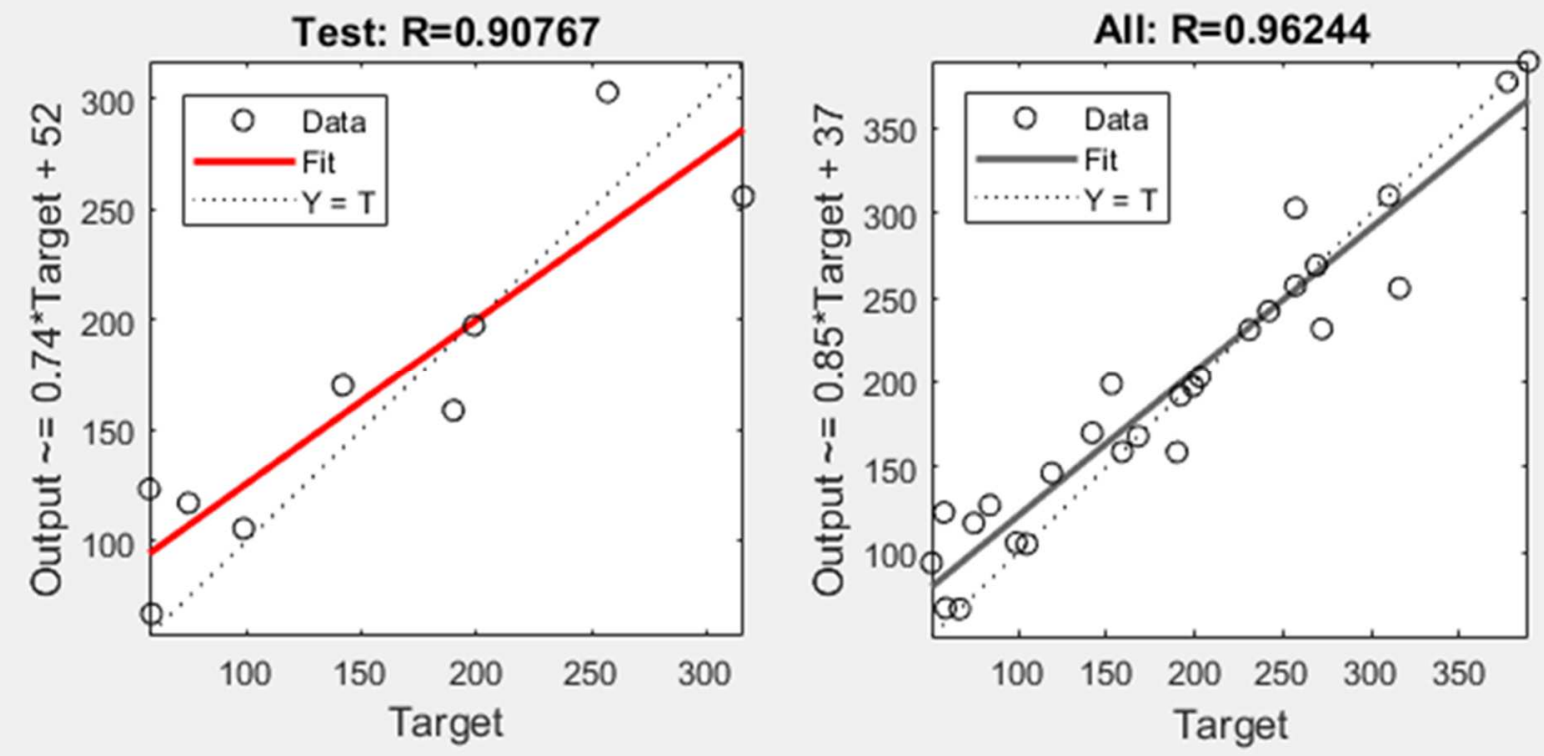

Figure 28: 45\% Training, 20\% Validation, 35\% Testing and 10 Hidden Neurons

70\% Training, 25\% Validation, 5\% Testing and 10 Hidden Neurons 

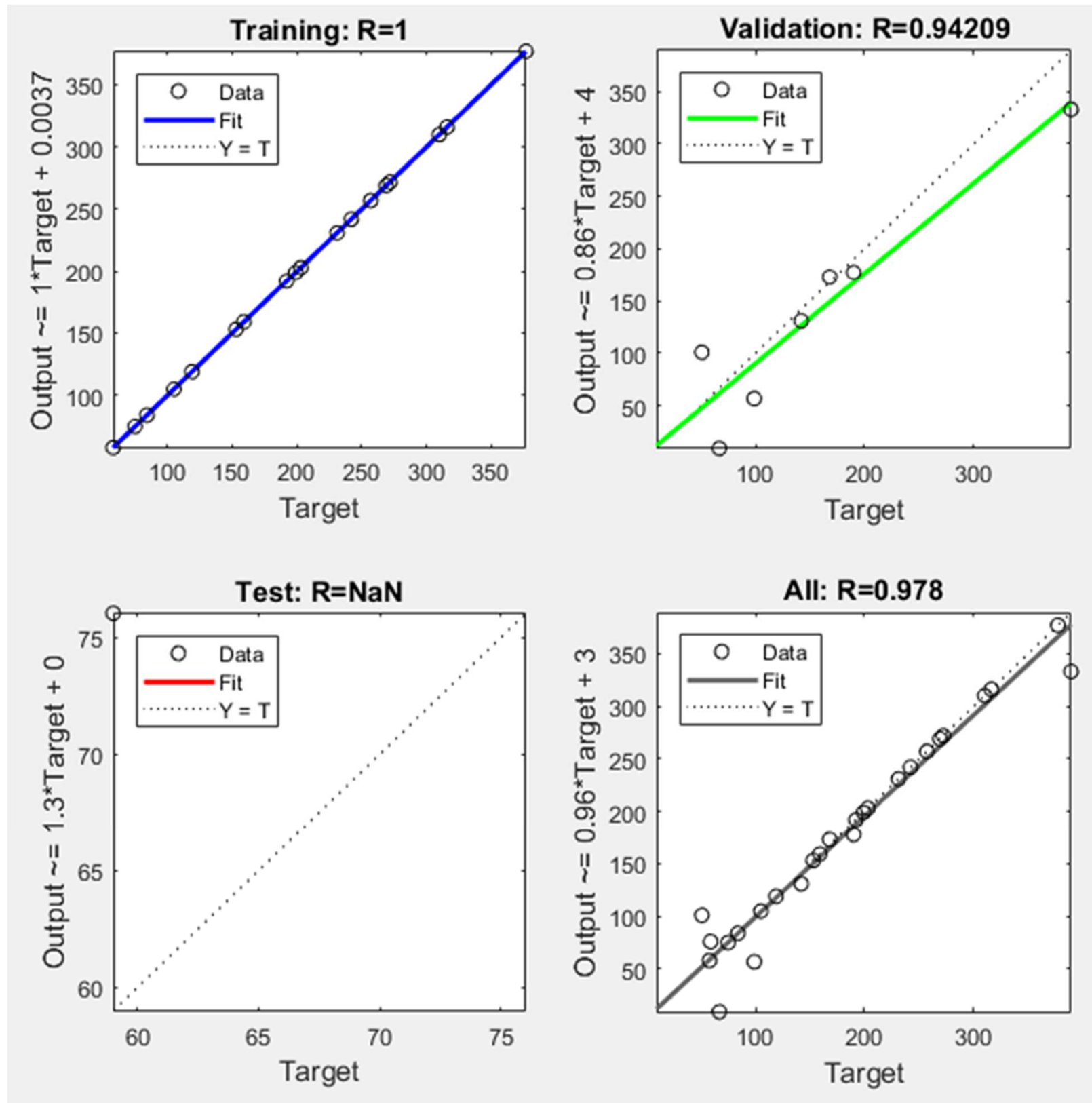

Figure 29: 70\% Training, 25\% Validation, 5\% Testing and 10 Hidden Neurons

\section{5\% Training, 25\% Validation, $10 \%$ Testing and 10 Hidden Neurons}

Figure 30 shows the regression plot for $65 \%$ training, $25 \%$ validation, $10 \%$ testing and 10 hidden neurons. This gives regression values of 0.99933 for training, 0.99569 for validation, 0.99829 for testing and 0.99775 for all. 

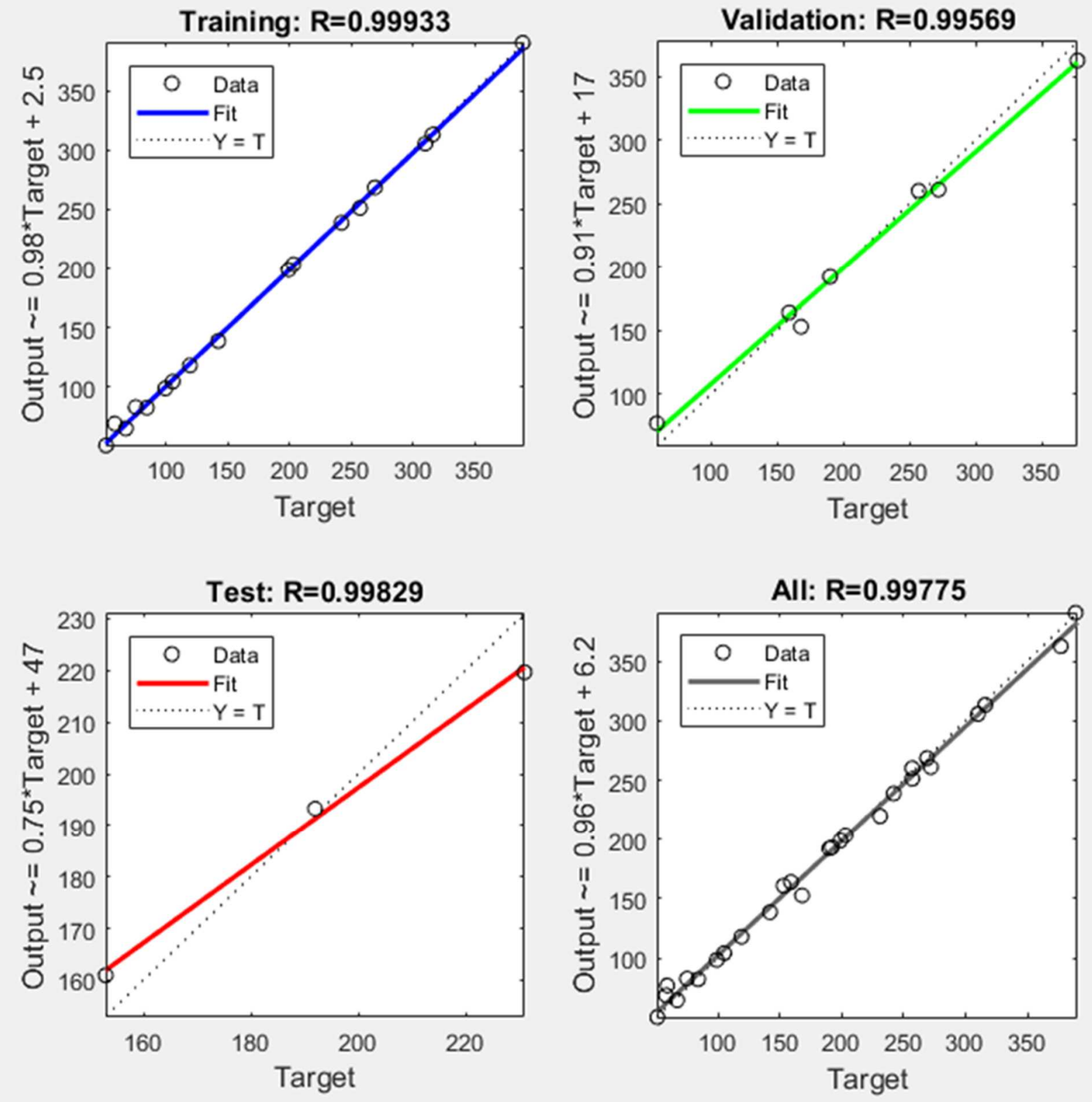

Figure 30: 65\% Training, 25\% Validation, 10\% Testing and 10 Hidden Neurons

$60 \%$ Training, 25\% Validation, $15 \%$ Testing and 10 Hidden Neurons

Figure 31 shows the regression plot for $60 \%$ training, $25 \%$ validation, $15 \%$ testing and 10 hidden neurons. This gives regression values of 1.0 for training, 0.94739 for validation, 0.96422 for testing and 0.97126 for all. 

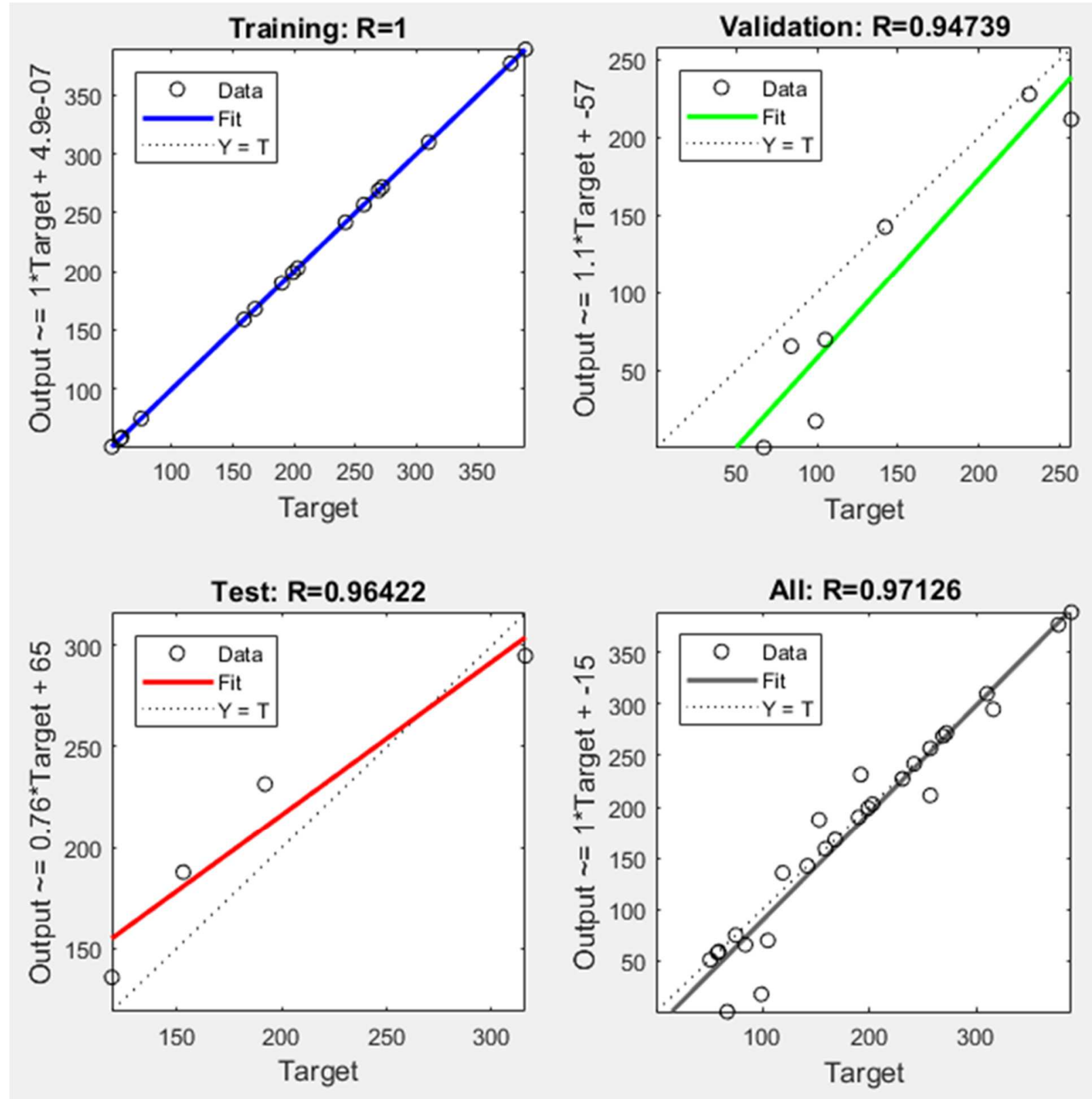

Figure 31: 60\% Training, 25\% Validation, 15\% Testing and 10 Hidden Neurons

55\% Training, 25\% Validation, 20\% Testing and 10 Hidden Neurons

Figure 32 shows the regression plot for 55\% training, 25\% validation, 20\% testing and 10 hidden neurons. This gives regression values of 1.0 for training, 0.98686 for validation, 0.98401 for testing and 0.99063 for all. 

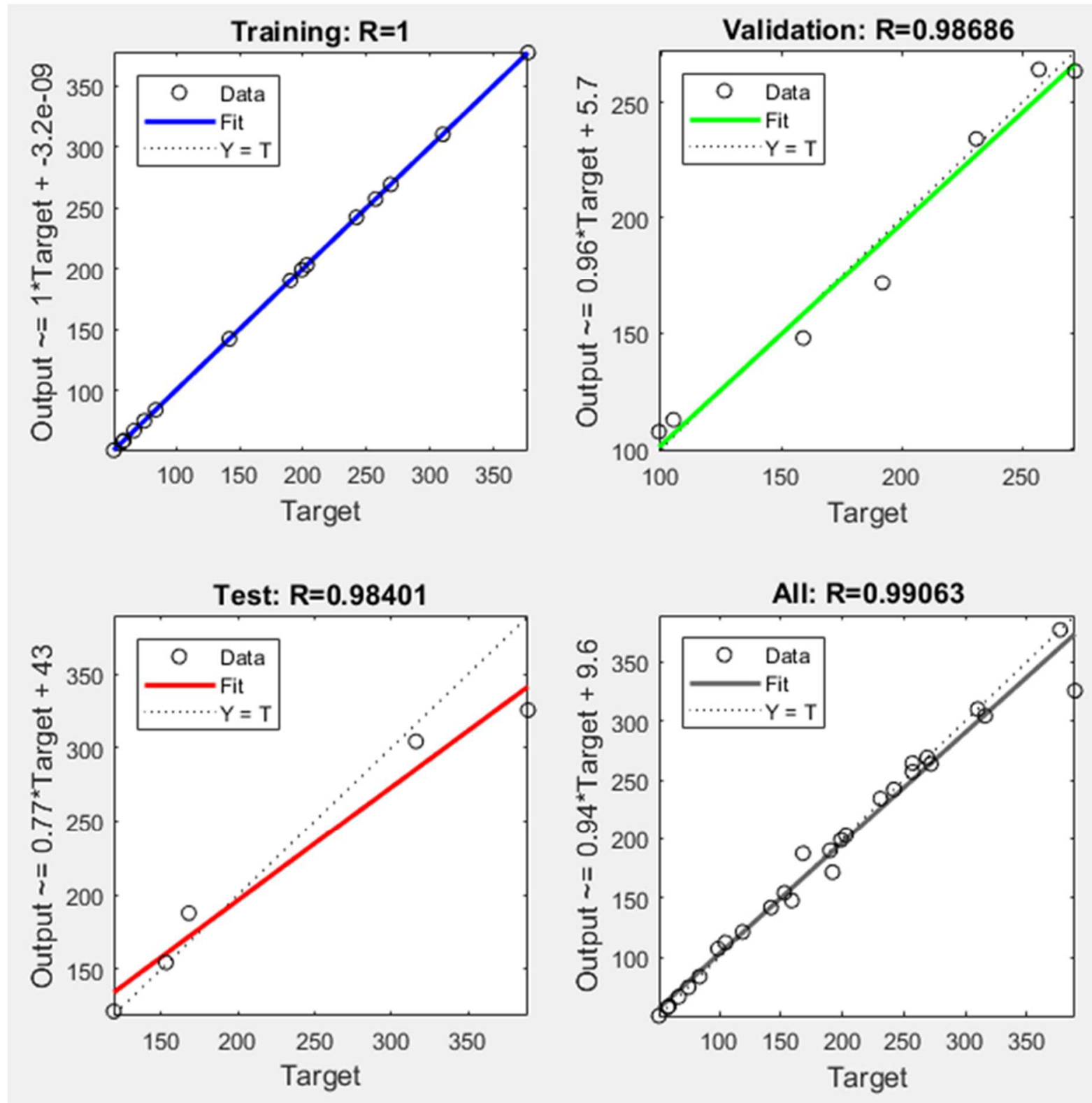

Figure 32: 55\% Training, 25\% Validation, 20\% Testing and 10 Hidden Neurons

50\% Training, 25\% Validation, 25\% Testing and 10 Hidden Neurons

Figure 33 shows the regression plot for 50\% training, 25\% validation, 25\% testing and 10 hidden neurons. This gives regression values of 1.0 for training, 0.99439 for validation, 0.98249 for testing and 0.99405 for all. 

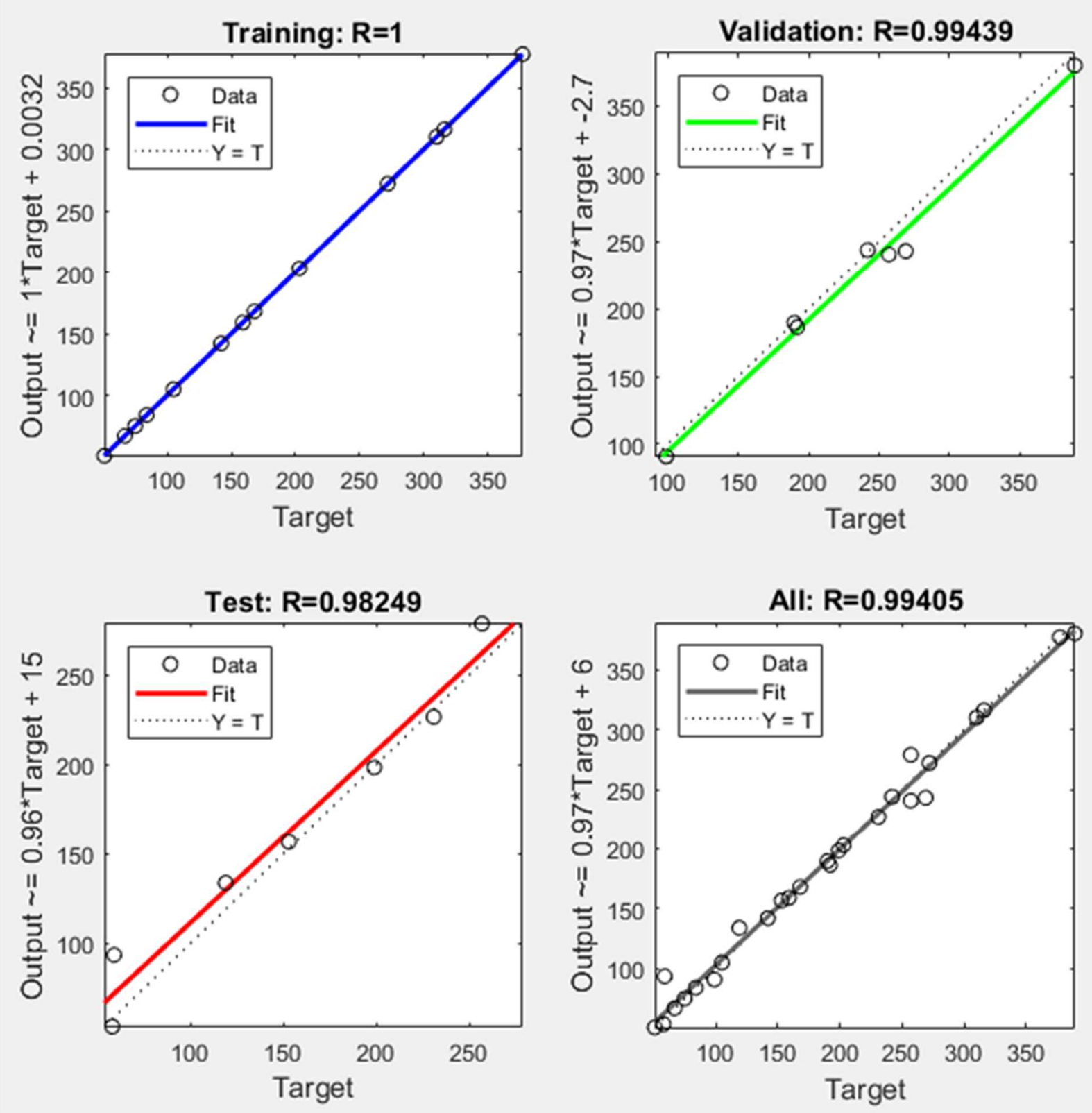

Figure 33: 50\% Training, 25\% Validation, 25\% Testing and 10 Hidden Neurons

\section{5\% Training, 25\% Validation, 30\% Testing and 10 Hidden Neurons}

Figure 34 shows the regression plot for $45 \%$ training, $25 \%$ validation, $30 \%$ testing and 10 hidden neurons. This gives regression values of 1.0 for training, 0.72784 for validation, 0.85004 for testing and 0.89834 for all. 

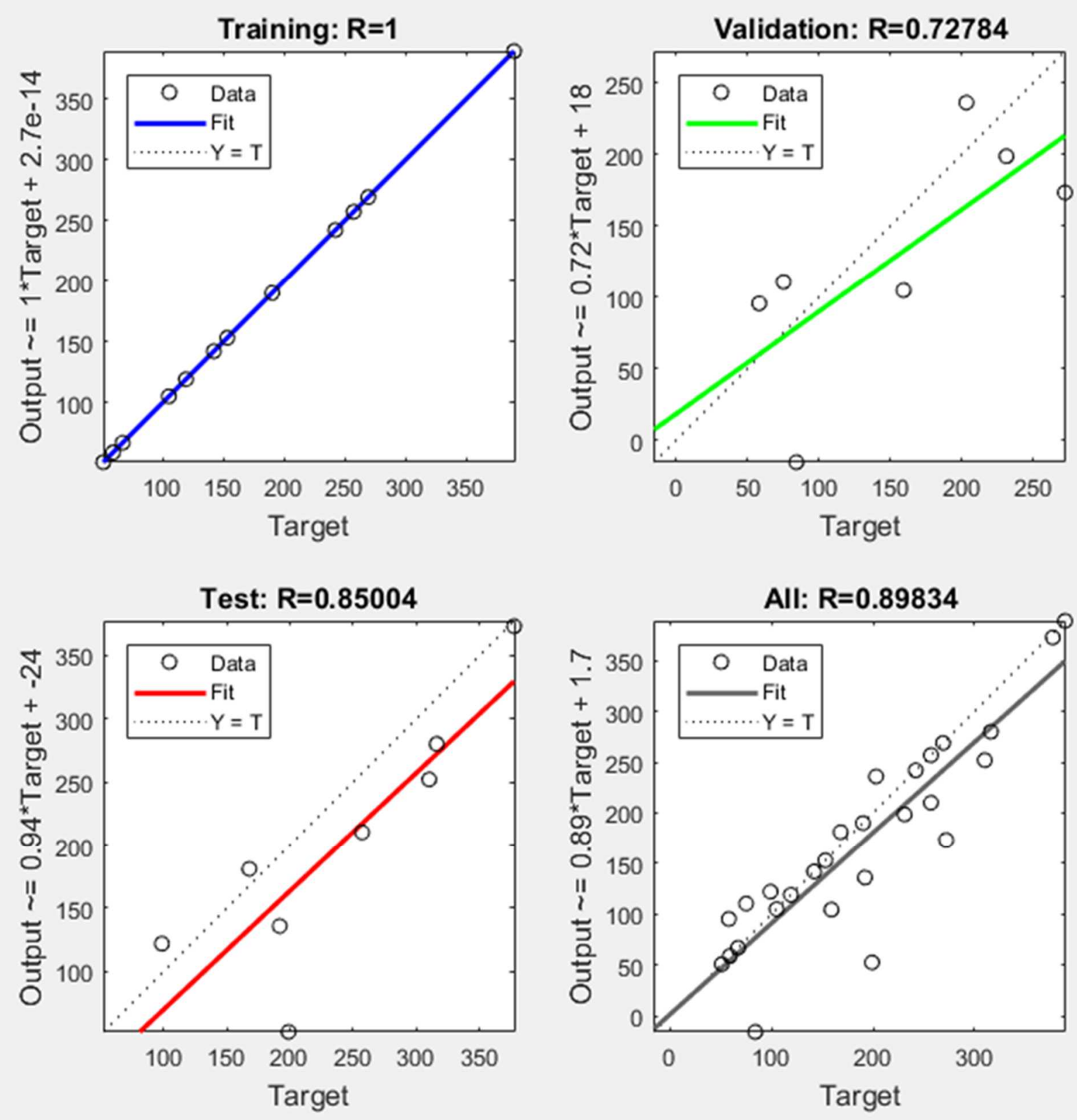

Figure 34: 45\% Training, 25\% Validation, 30\% Testing and 10 Hidden Neurons

40\% Training, 25\% Validation, 35\% Testing and 10 Hidden Neurons

Figure 35 shows the regression plot for $40 \%$ training, 25\% validation, $35 \%$ testing and 10 hidden neurons. This gives regression values of 1.0 for training, 0.7163 for validation, 0.93581 for testing and 0.84267 for all. 

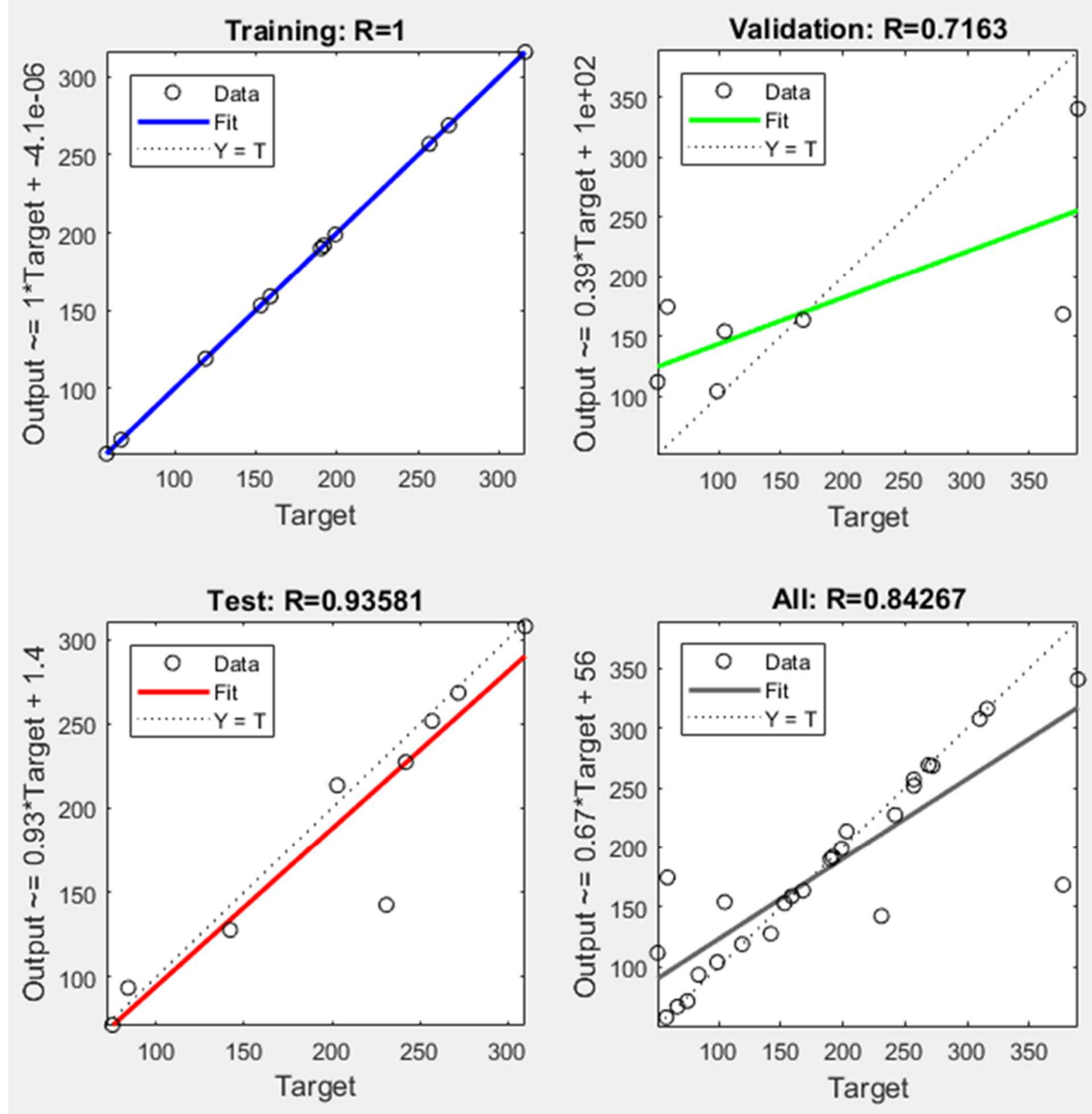

Figure 35: 40\% Training, 25\% Validation, 35\% Testing and 10 Hidden Neurons 65\% Training, 30\% Validation, 5\% Testing and 10 Hidden Neurons 


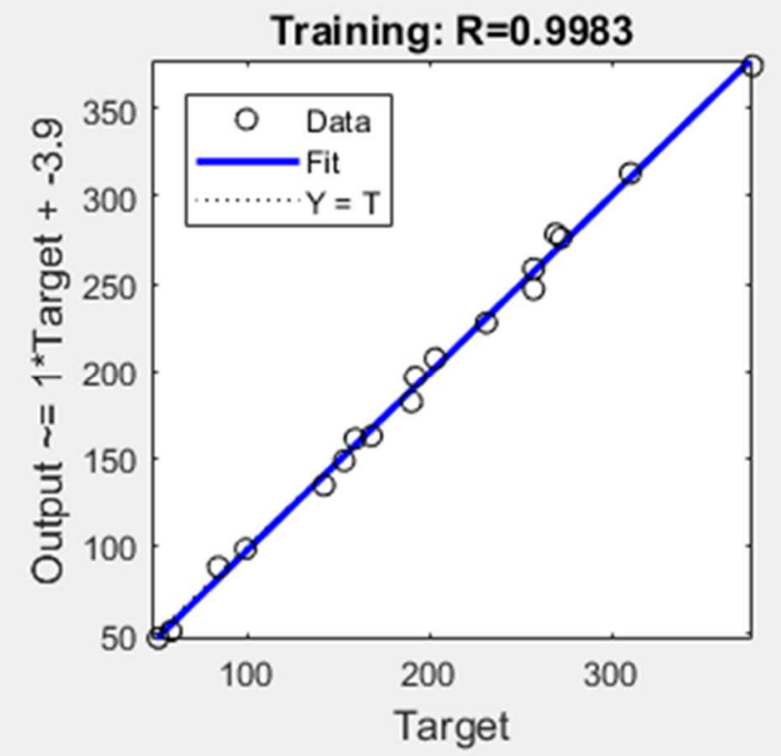

Test: $\mathrm{R}=\mathrm{NaN}$

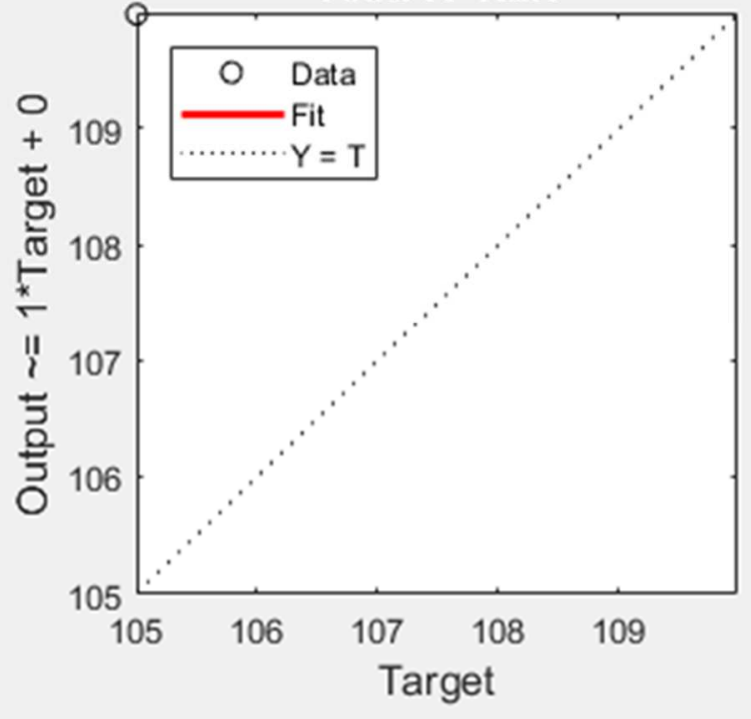

Validation: $\mathbf{R}=\mathbf{0 . 9 6 0 8 9}$

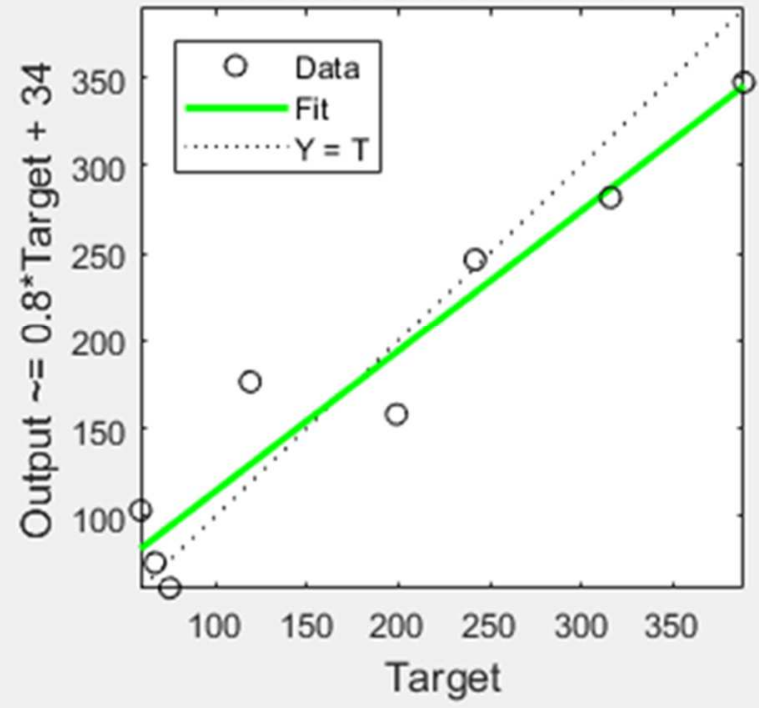

All: $\mathbf{R}=\mathbf{0 . 9 7 9 3}$

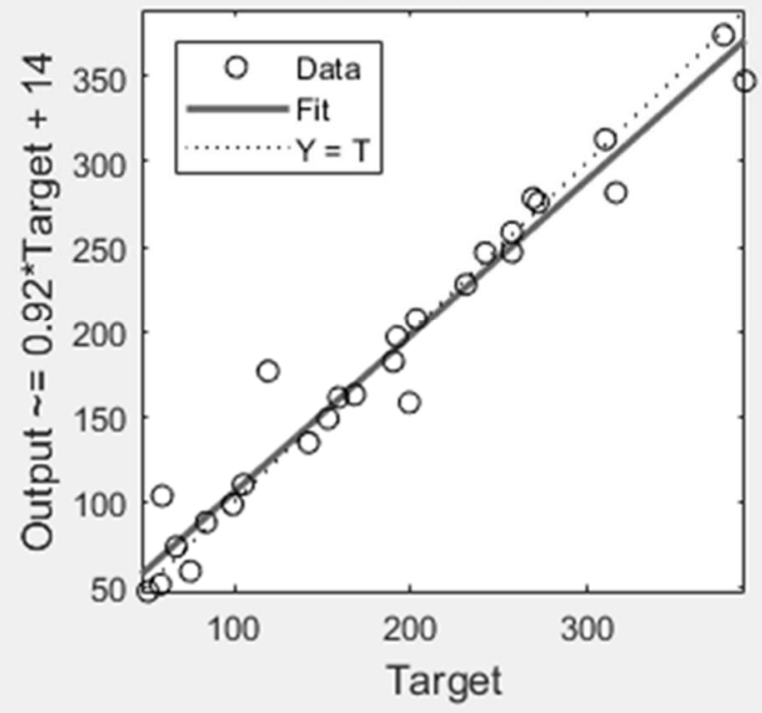

Figure 36: 65\% Training, 30\% Validation, 5\% Testing and 10 Hidden Neurons

60\% Training, 30\% Validation, 10\% Testing and 10 Hidden Neurons

Figure 37 shows the regression plot for 60\% training, 30\% validation, $10 \%$ testing and 10 hidden neurons. This gives regression values of 0.99988 for training, 0.97554 for validation, 0.96485 for testing and 0.9906 for all. 

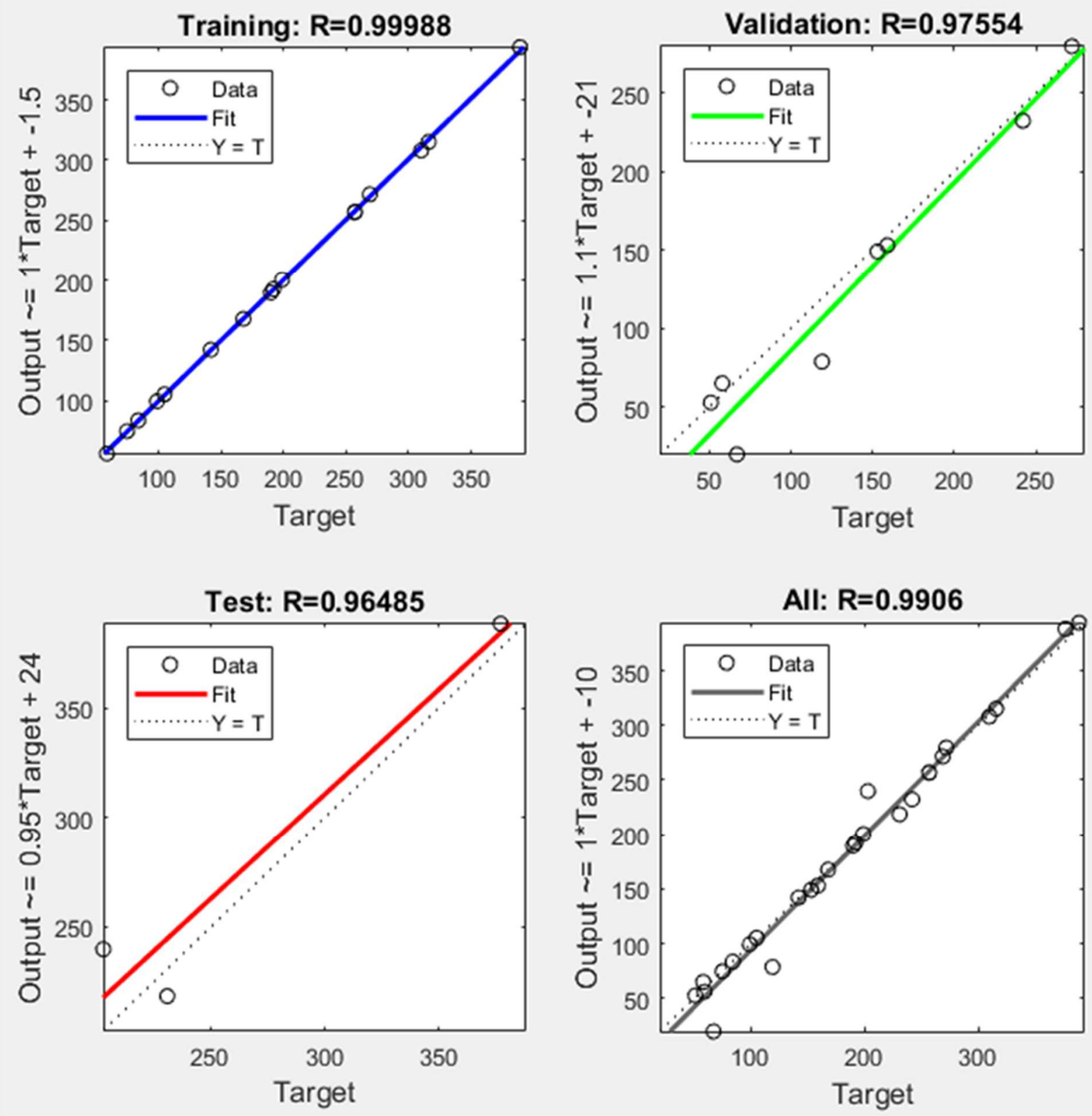

Figure 37: 60\% Training, 30\% Validation, 10\% Testing and 10 Hidden Neurons

55\% Training, 30\% Validation, 15\% Testing and 10 Hidden Neurons

Figure 38 shows the regression plot for 55\% training, 30\% validation, $15 \%$ testing and 10 hidden neurons. This gives regression values of 1.0 for training, 0.91483 for validation, 0.99984 for testing and 0.98548 for all. 


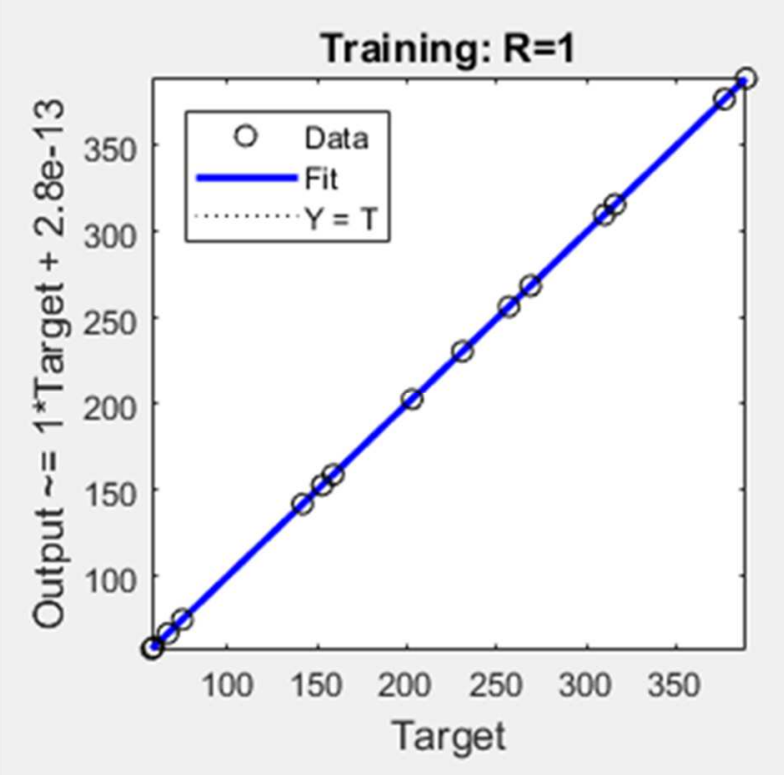

Test: $\mathbf{R}=\mathbf{0 . 9 9 9 8 4}$

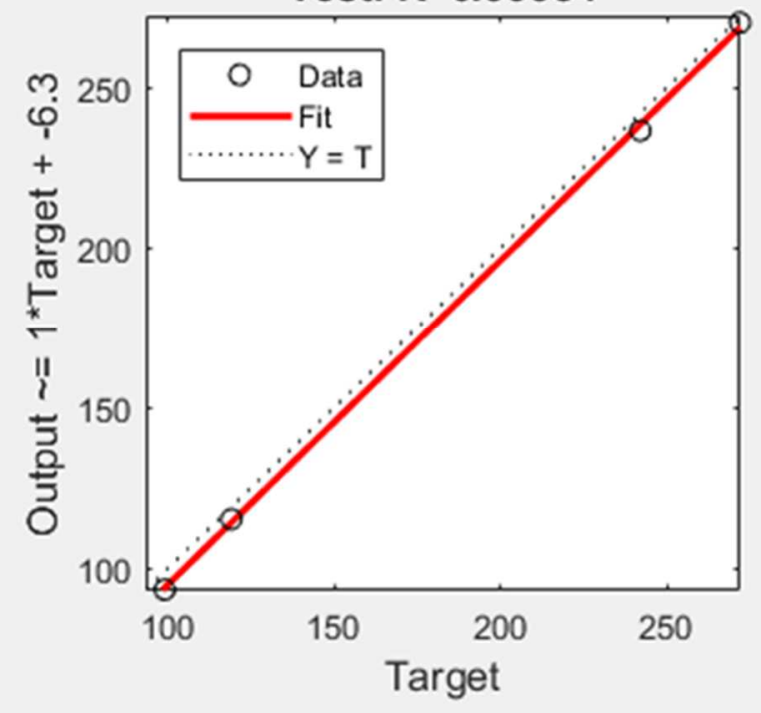

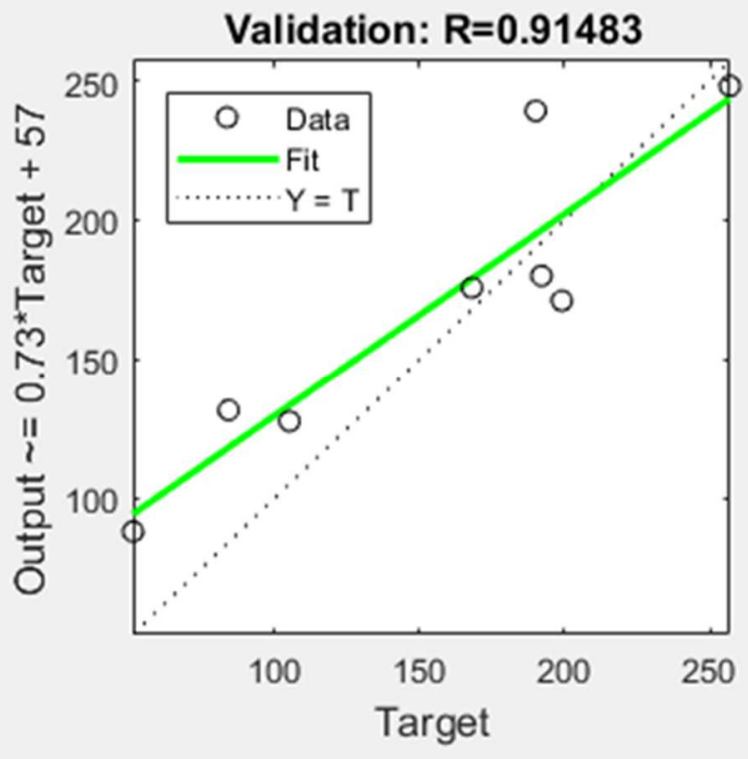

All: $\mathbf{R}=\mathbf{0 . 9 8 5 4 8}$

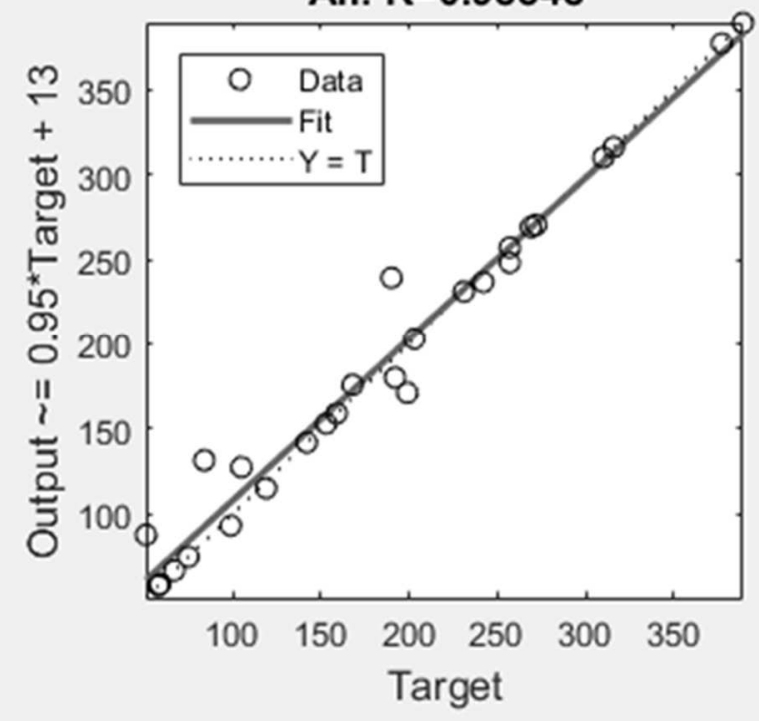

Figure 38: 55\% Training, 30\% Validation, 15\% Testing and 10 Hidden Neurons

50\% Training, 30\% Validation, 20\% Testing and 10 Hidden Neurons

Figure 39 shows the regression plot for 50\% training, 30\% validation, 20\% testing and 10 hidden neurons. This gives regression values of 1.0 for training, 0.90298 for validation, 0.99075 for testing and 0.95045 for all. 

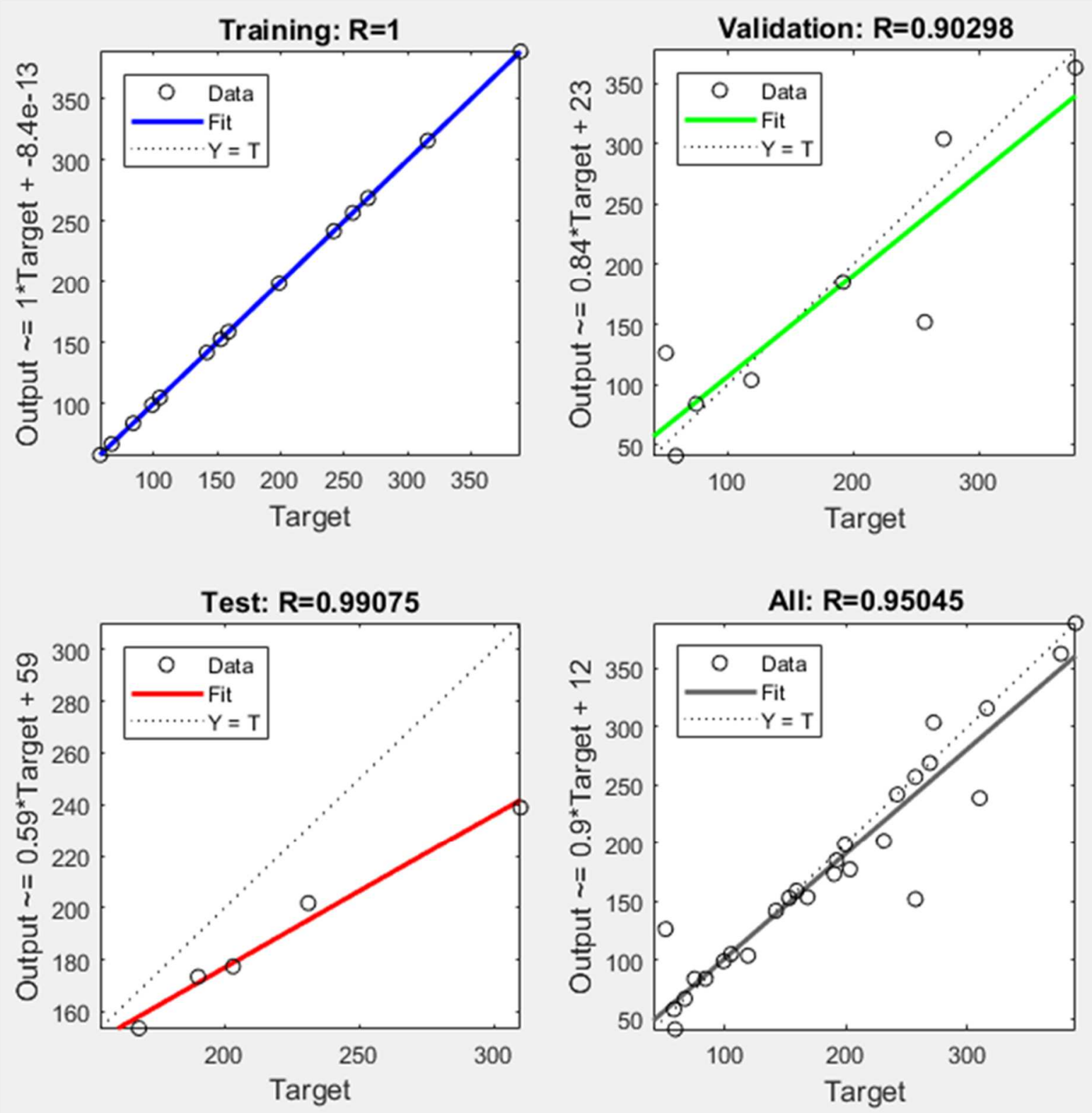

Figure 39: 50\% Training, 30\% Validation, 20\% Testing and 10 Hidden Neurons

45\% Training, 30\% Validation, 25\% Testing and 10 Hidden Neurons

Figure 40 shows the regression plot for $45 \%$ training, 30\% validation, $25 \%$ testing and 10 hidden neurons. This gives regression values of 1.0 for training, 0.89397 for validation, 0.94592 for testing and 0.96783 for all. 

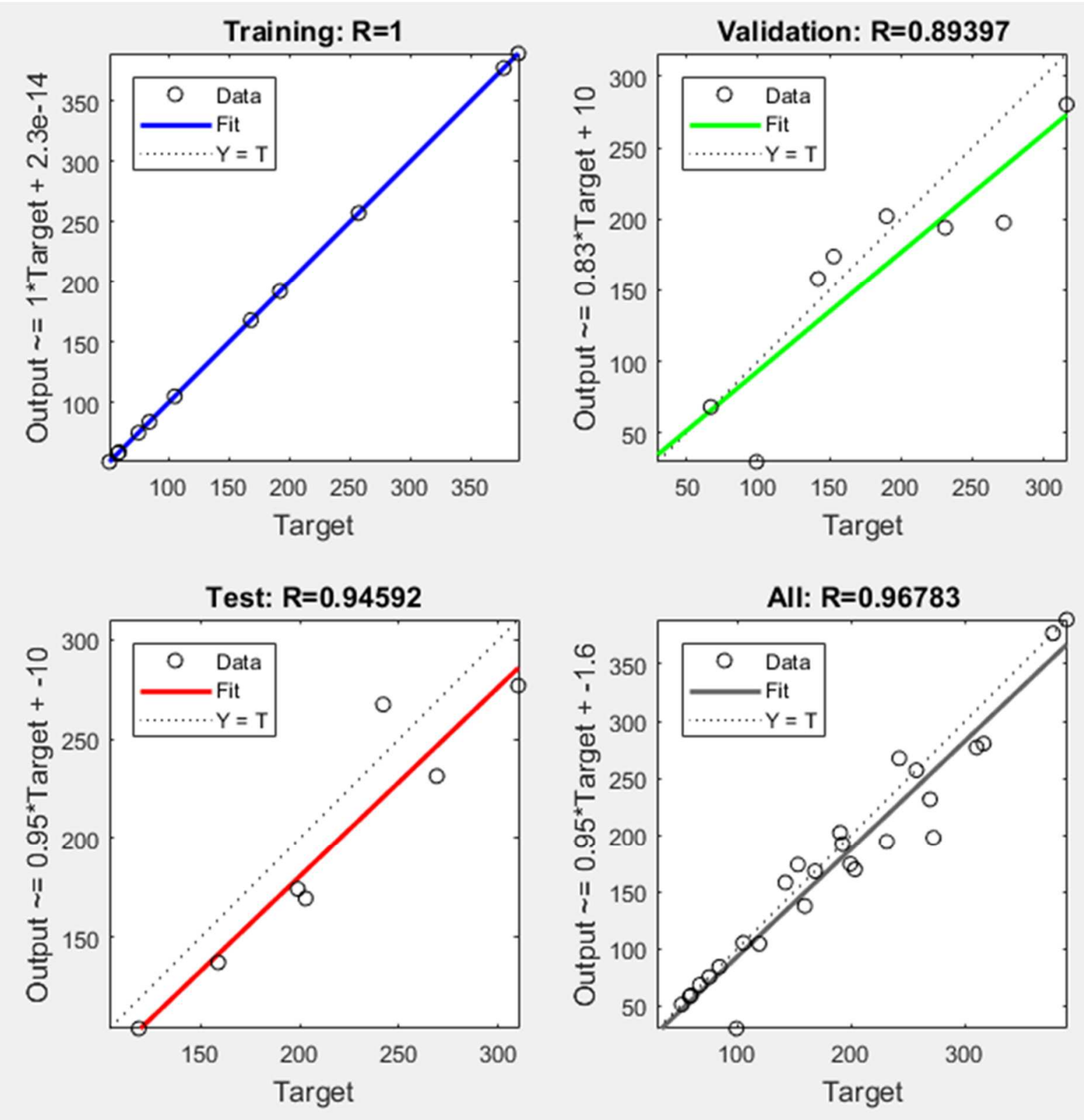

Figure 40: 45\% Training, 30\% Validation, 25\% Testing and 10 Hidden Neurons

40\% Training, 30\% Validation, 30\% Testing and 10 Hidden Neurons

Figure 41 shows the regression plot for $40 \%$ training, 30\% validation, 30\% testing and 10 hidden neurons. This gives regression values of 0.99987 for training, 0.95759 for validation, 0.93432 for testing and 0.97175 for all. 

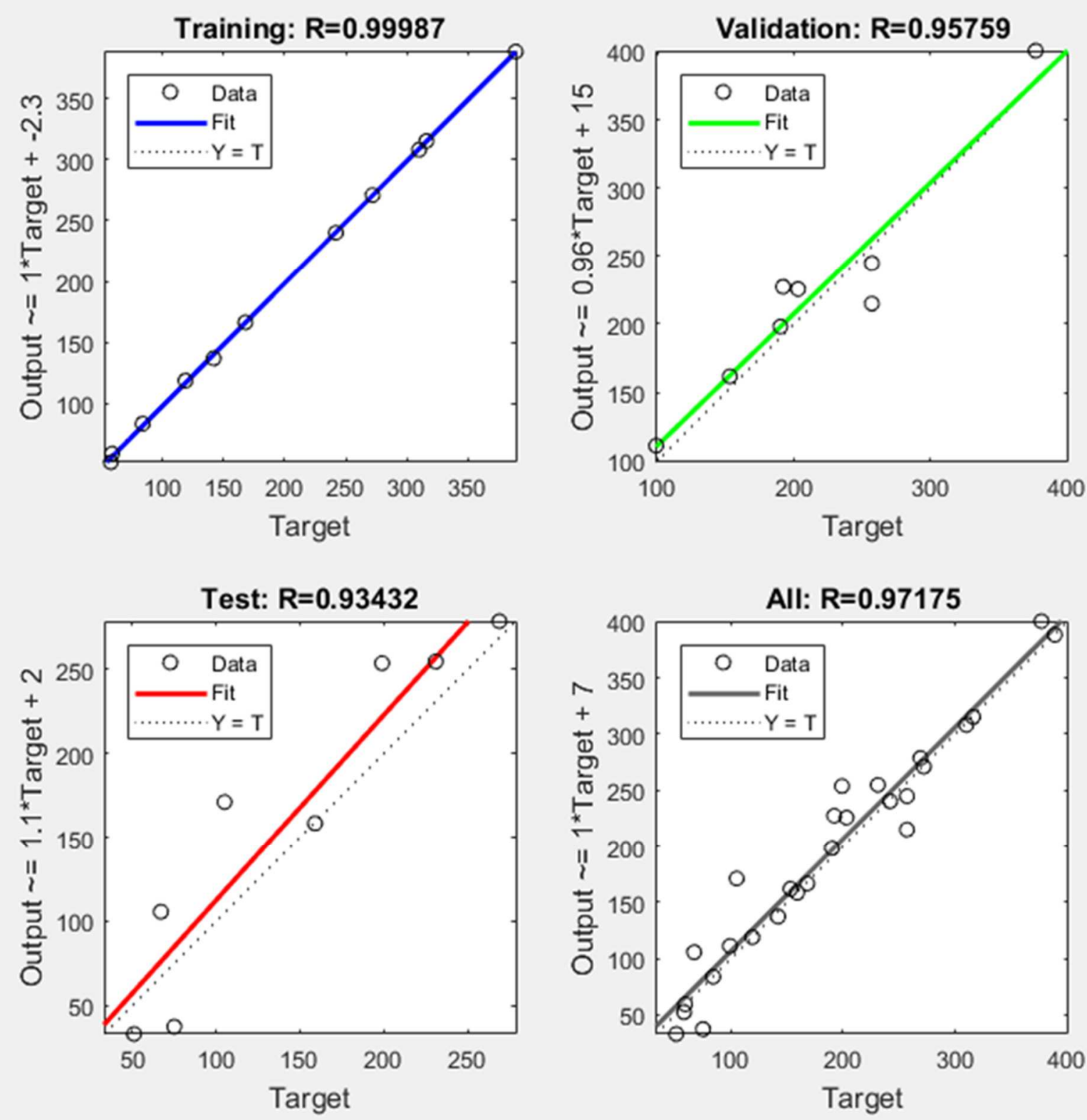

Figure 41: 40\% Training, 30\% Validation, 30\% Testing and 10 Hidden Neurons

\section{5\% Training, 30\% Validation, 35\% Testing and 10 Hidden Neurons}

Figure 42 shows the regression plot for 35\% training, 30\% validation, 35\% testing and 10 hidden neurons. This gives regression values of 1.0 for training, 0.9207 for validation, 0.97444 for testing and 0.96778 for all. 

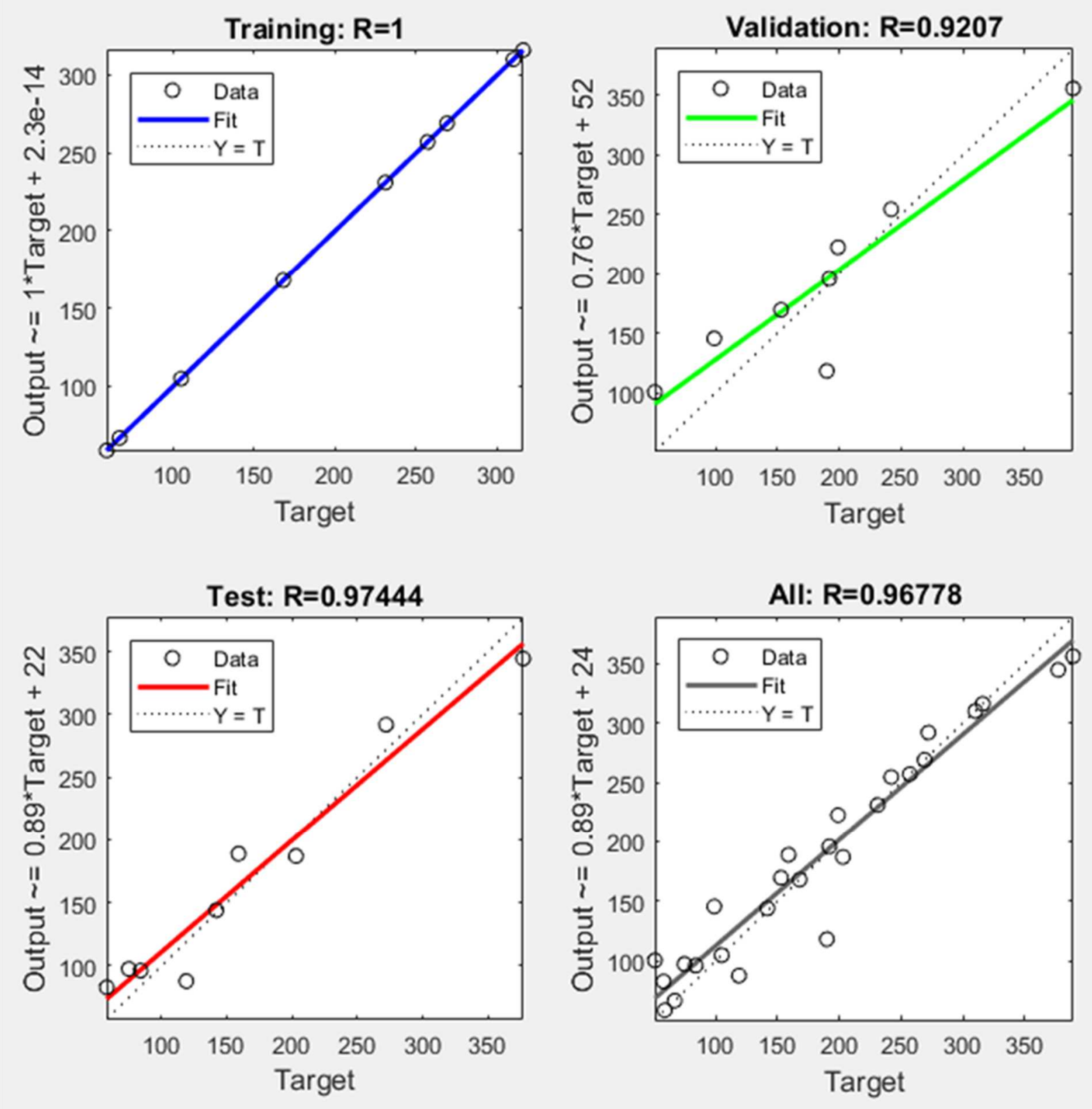

Figure 42: 35\% Training, 30\% Validation, 35\% Testing and 10 Hidden Neurons $60 \%$ Training, 35\% Validation, 5\% Testing and 10 Hidden Neurons 

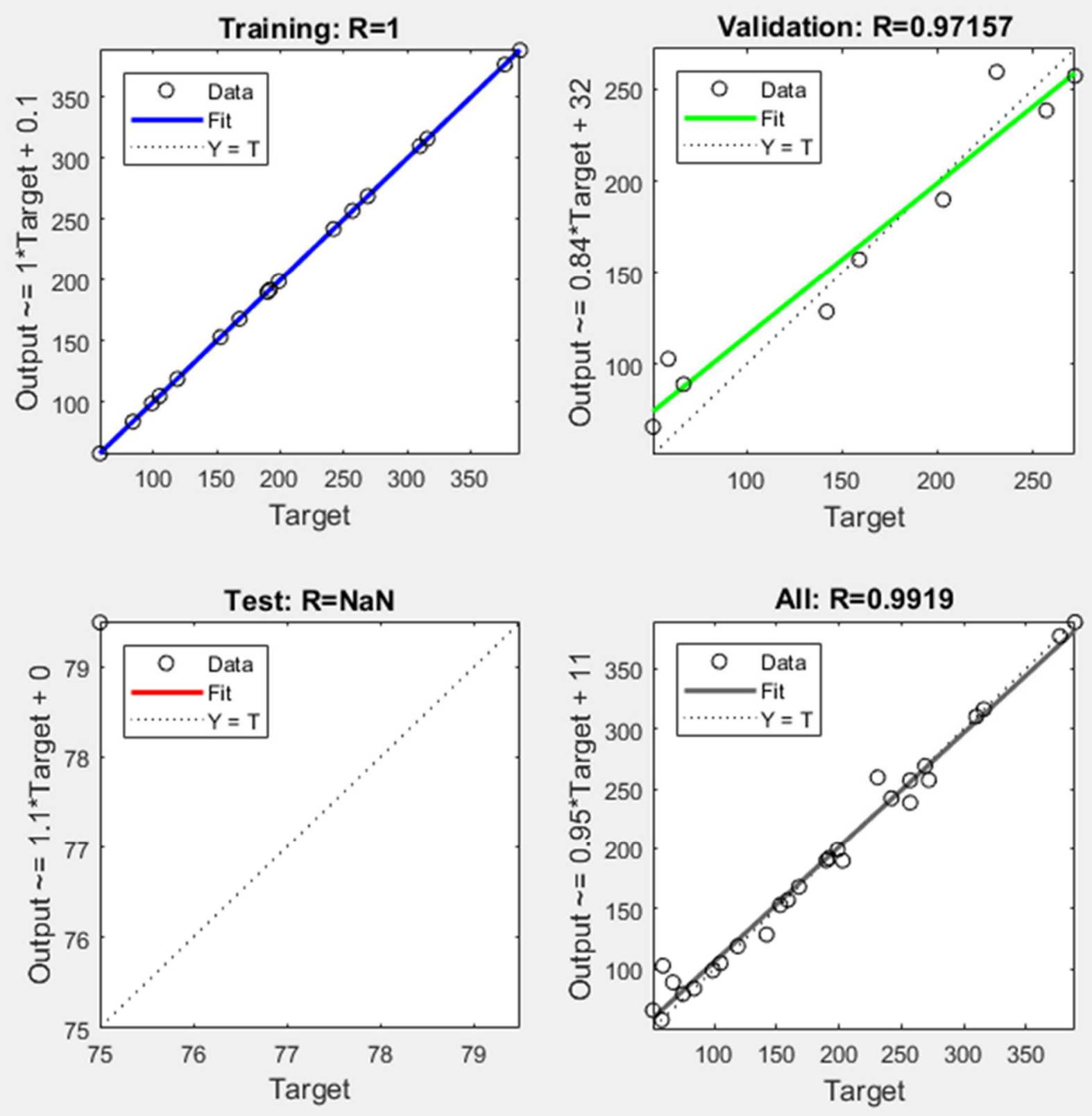

Figure 43: 60\% Training, 35\% Validation, 5\% Testing and 10 Hidden Neurons

\section{5\% Training, 35\% Validation, 10\% Testing and 10 Hidden Neurons}

Figure 44 shows the regression plot for 55\% training, 35\% validation, $10 \%$ testing and 10 hidden neurons. This gives regression values of 1.0 for training, 0.94183 for validation, 0.99593 for testing and 0.96768 for all. 

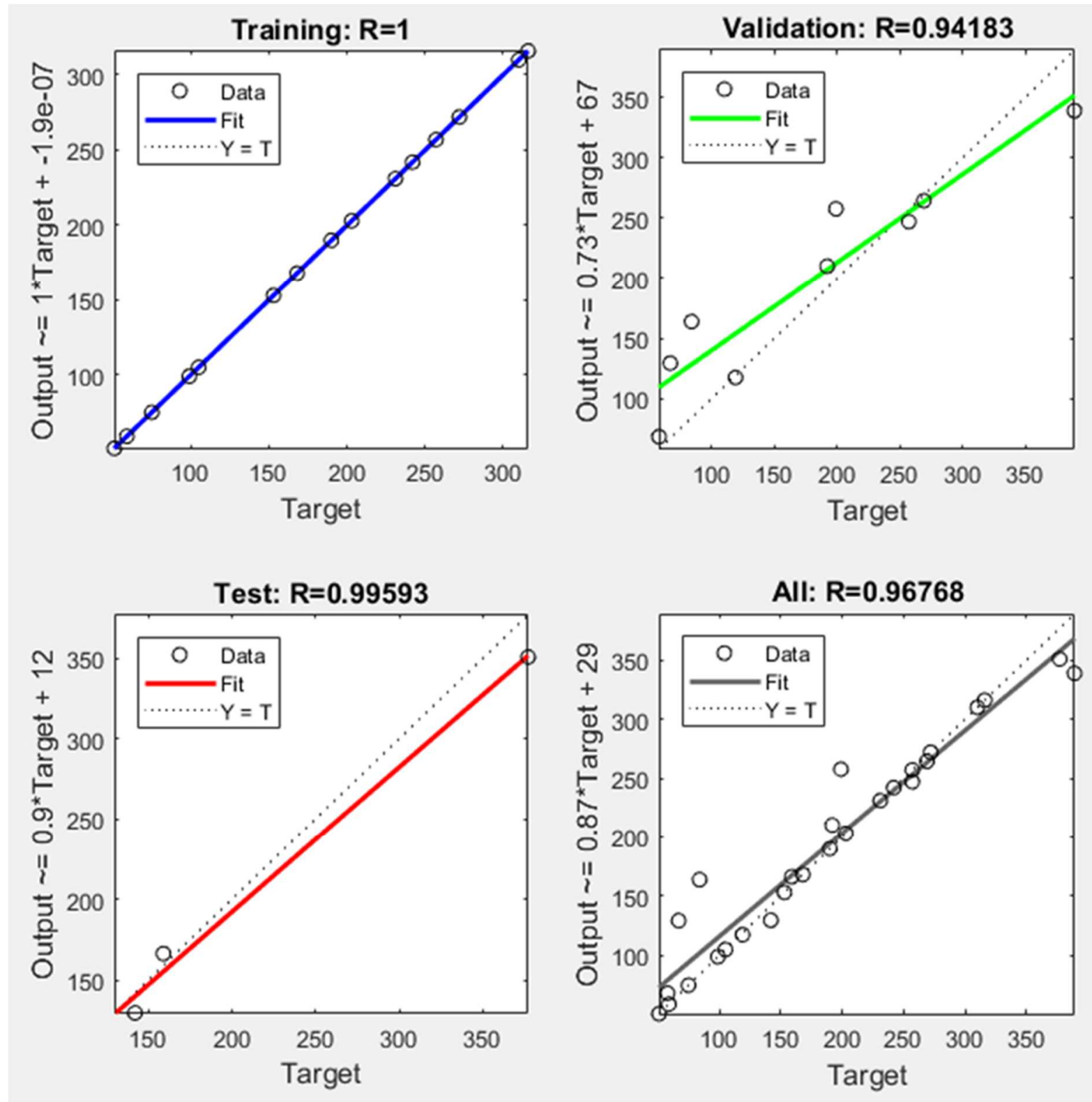

Figure 44: 55\% Training, 35\% Validation, 10\% Testing and 10 Hidden Neurons

\section{0\% Training, 35\% Validation, 15\% Testing and 10 Hidden Neurons}

Figure 45 shows the regression plot for 50\% training, 35\% validation, $15 \%$ testing and 10 hidden neurons. This gives regression values of 1.0 for training, 0.95488 for validation, 0.99625 for testing and 0.98075 for all. 

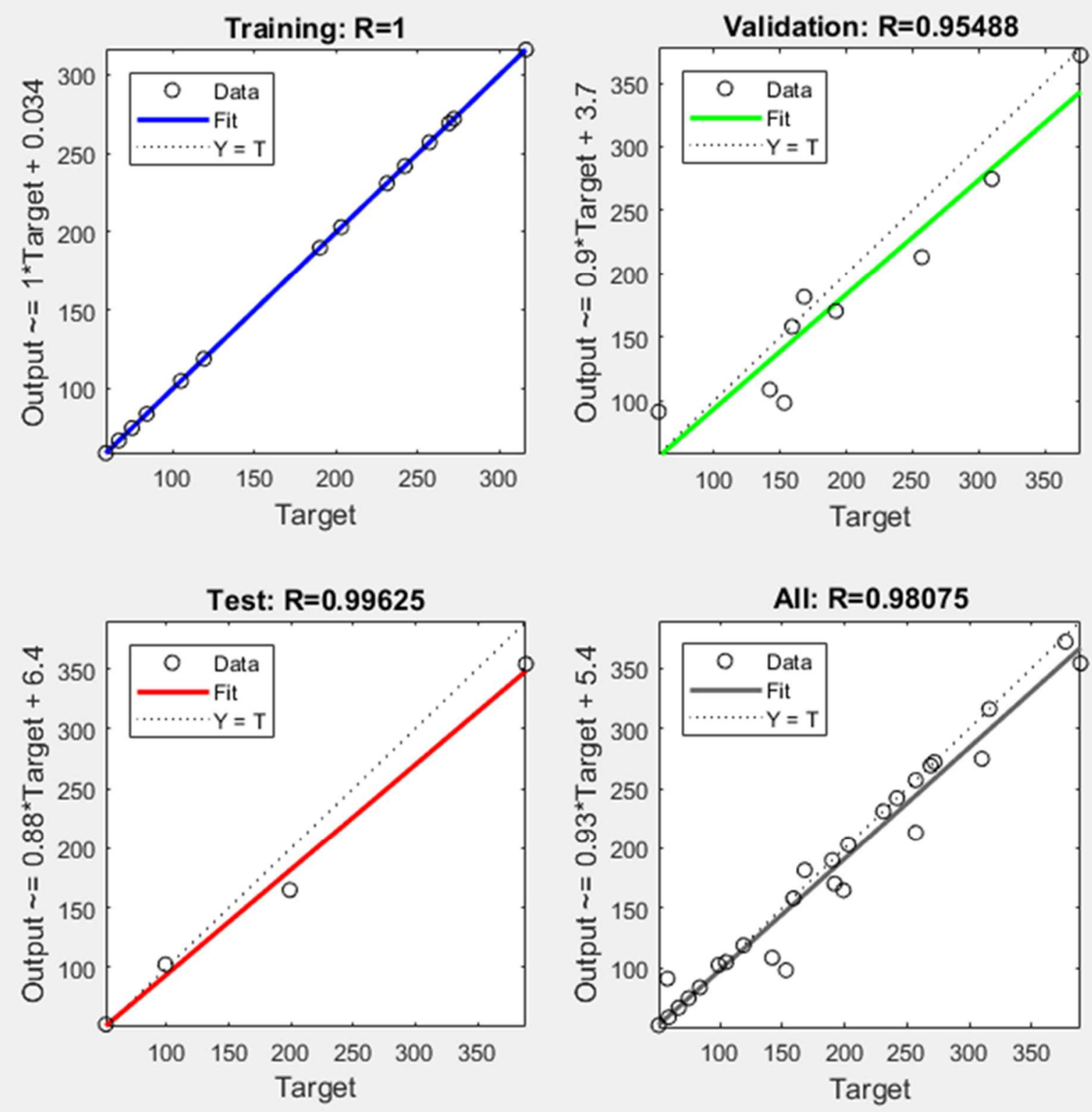

Figure 45: 50\% Training, 35\% Validation, 15\% Testing and 10 Hidden Neurons

\section{5\% Training, 35\% Validation, 20\% Testing and 10 Hidden Neurons}

Figure 46 shows the regression plot for $45 \%$ training, 35\% validation, $20 \%$ testing and 10 hidden neurons. This gives regression values of 1.0 for training, 0.95585 for validation, 0.99582 for testing and 0.96945 for all. 

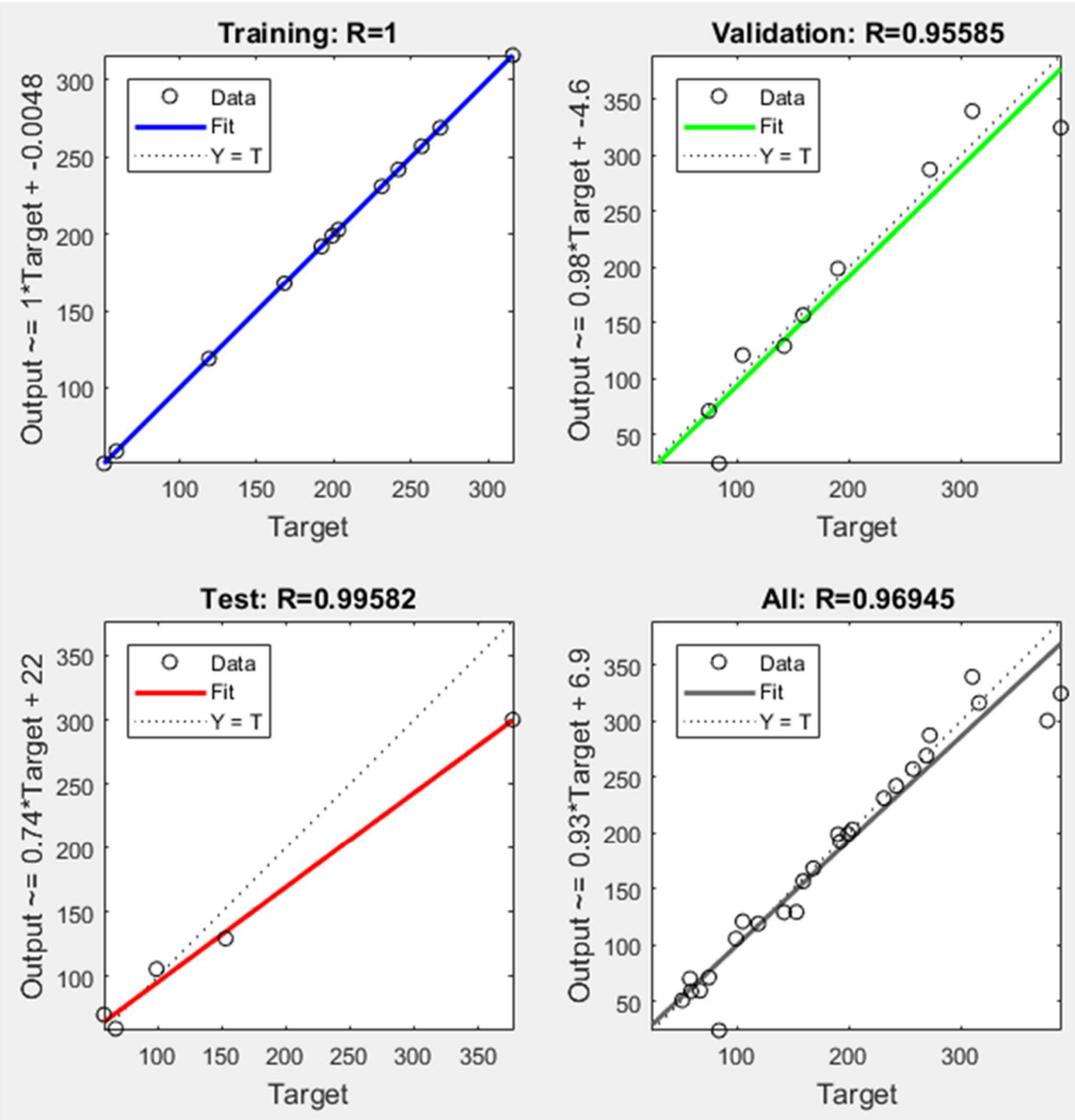

Figure 46: 45\% Training, 35\% Validation, 20\% Testing and 10 Hidden Neurons

40\% Training, 35\% Validation, 25\% Testing and 10 Hidden Neurons

Figure 47 shows the regression plot for $40 \%$ training, 35\% validation, $25 \%$ testing and 10 hidden neurons. This gives regression values of 0.99994 for training, 0.97579 for validation, 0.95444 for testing and 0.98319 for all. 

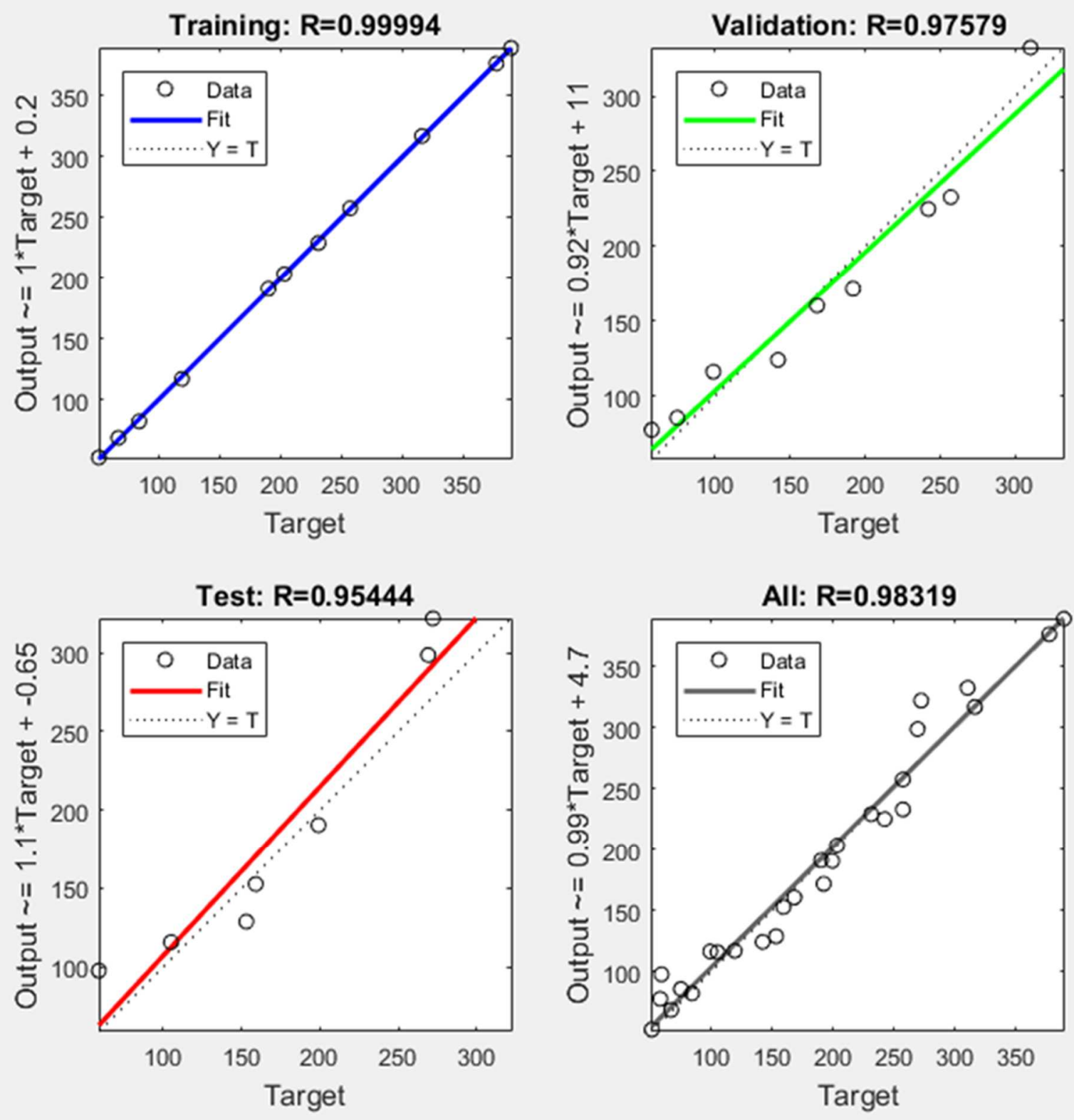

Figure 47: 40\% Training, 35\% Validation, 25\% Testing and 10 Hidden Neurons

35\% Training, 35\% Validation, 30\% Testing and 10 Hidden Neurons

Figure 48 shows the regression plot for 35\% training, 35\% validation, 30\% testing and 10 hidden neurons. This gives regression values of 1.0 for training, 0.91446 for validation, 0.98343 for testing and 0.96175 for all. 

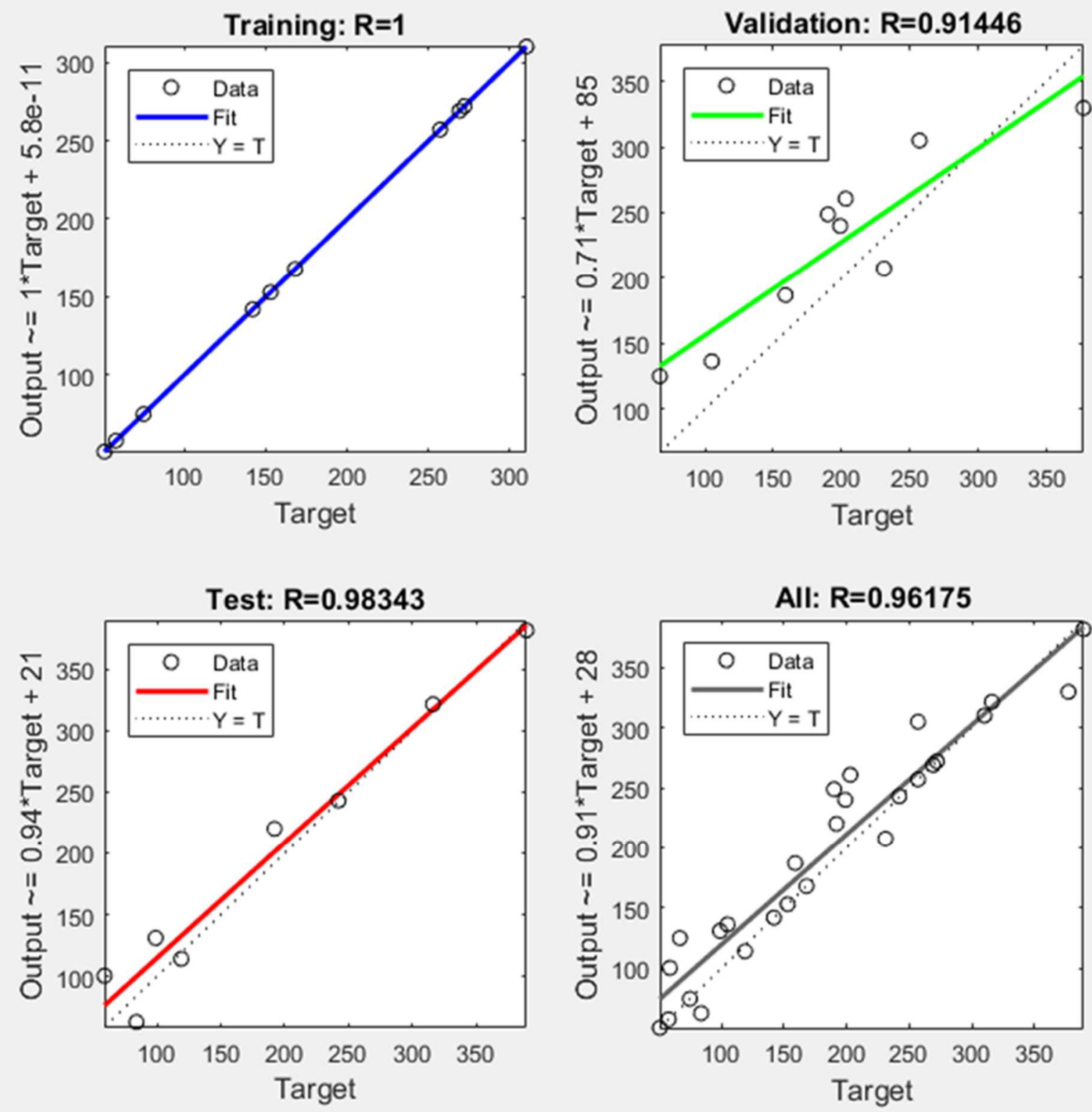

Figure 48: 35\% Training, 35\% Validation, 30\% Testing and 10 Hidden Neurons

30\% Training, 35\% Validation, 35\% Testing and 10 Hidden Neurons

Figure 49 shows the regression plot for 30\% training, 35\% validation, 35\% testing and 10 hidden neurons. This gives regression values of 1.0 for training, 0.8902 for validation, 0.94706 for testing and 0.93613 for all. 

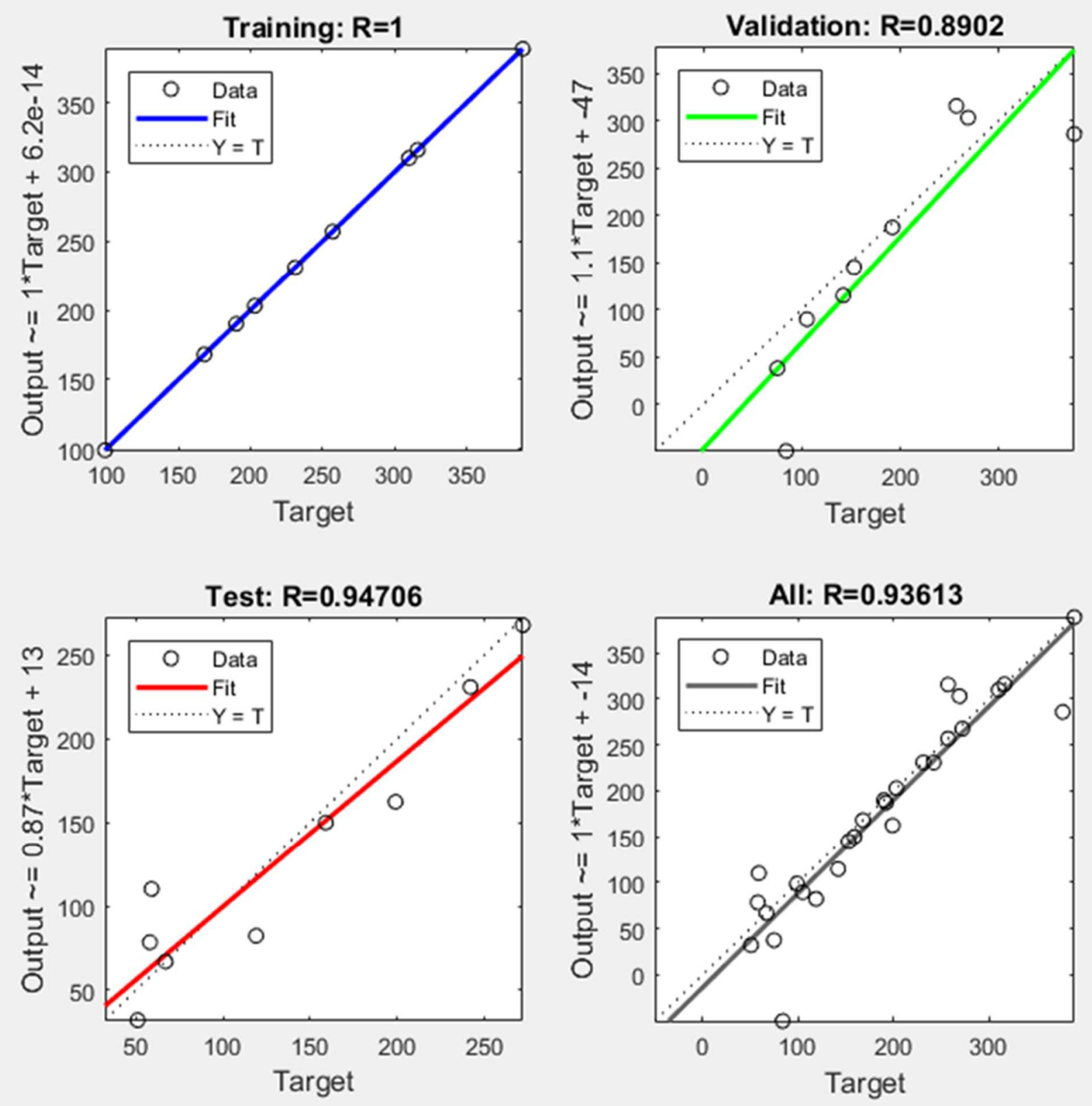

Figure 49: 30\% Training, 35\% Validation, 35\% Testing and 10 Hidden Neurons

\section{Varying Number of Hidden Neurons in Best Runs}

In addition to the previous 49 runs varying the training $\%$, validation $\%$, testing $\%$ and keeping the hidden neurons at 10 I ran 6 more combinations of the best 3 regressions using 5 and 15 hidden neurons for each. The top 3 runs came from run 10,16 and 23 . When changing the hidden neurons to 5 
and then $15 \mathrm{I}$ discovered that the $\mathrm{R}$ value was lower for all 6 run than at the original 10 hidden neurons. The following figure, figures 50-55, are the results I received from the Neural Network Fitting application.

75\% Training, $10 \%$ Validation, $15 \%$ Testing and 5 Hidden Neurons
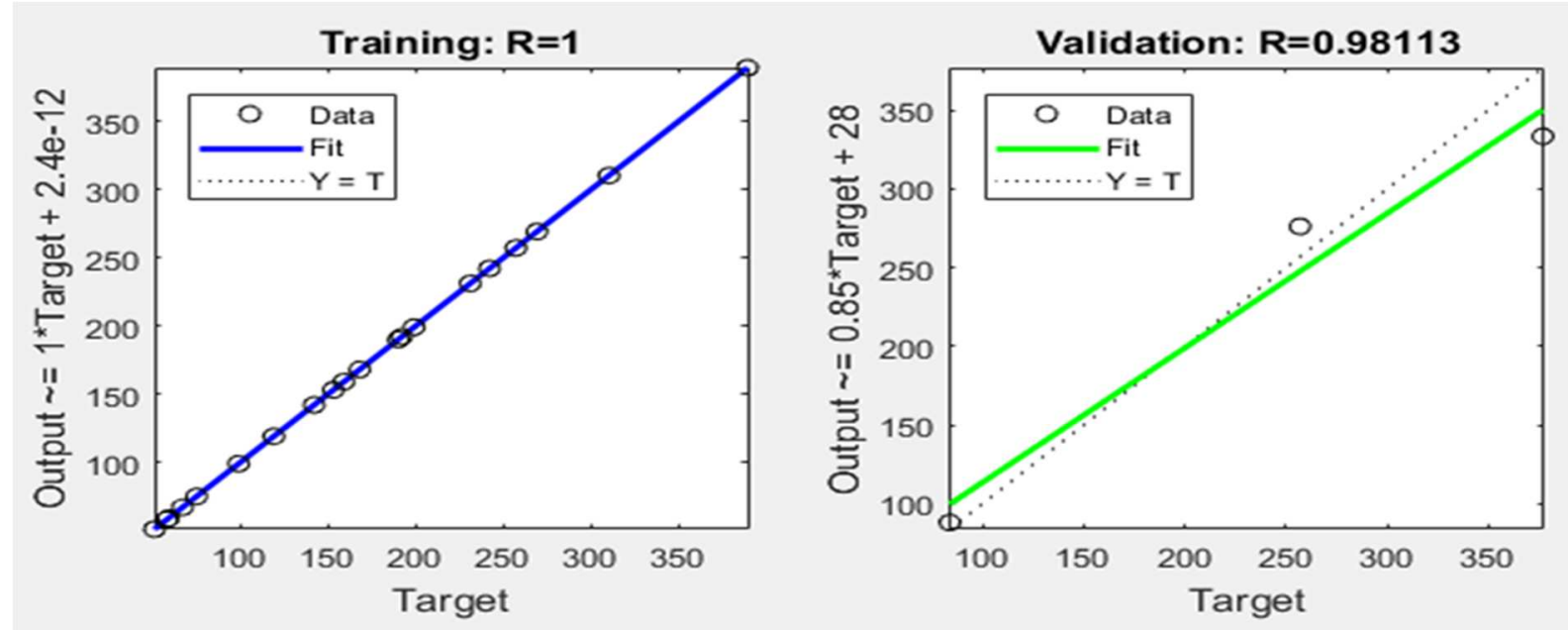

Test: $\mathbf{R}=\mathbf{0 . 9 9 9 4 1}$

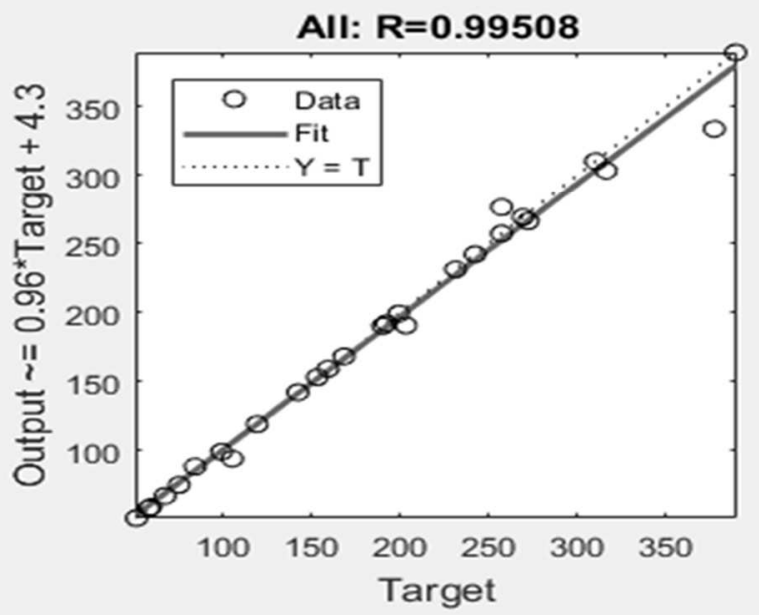

Figure 50: 75\% Training, 10\% Validation, 15\% Testing and 5 Hidden Neurons

75\% Training, 10\% Validation, 15\% Testing and 15 Hidden Neurons 

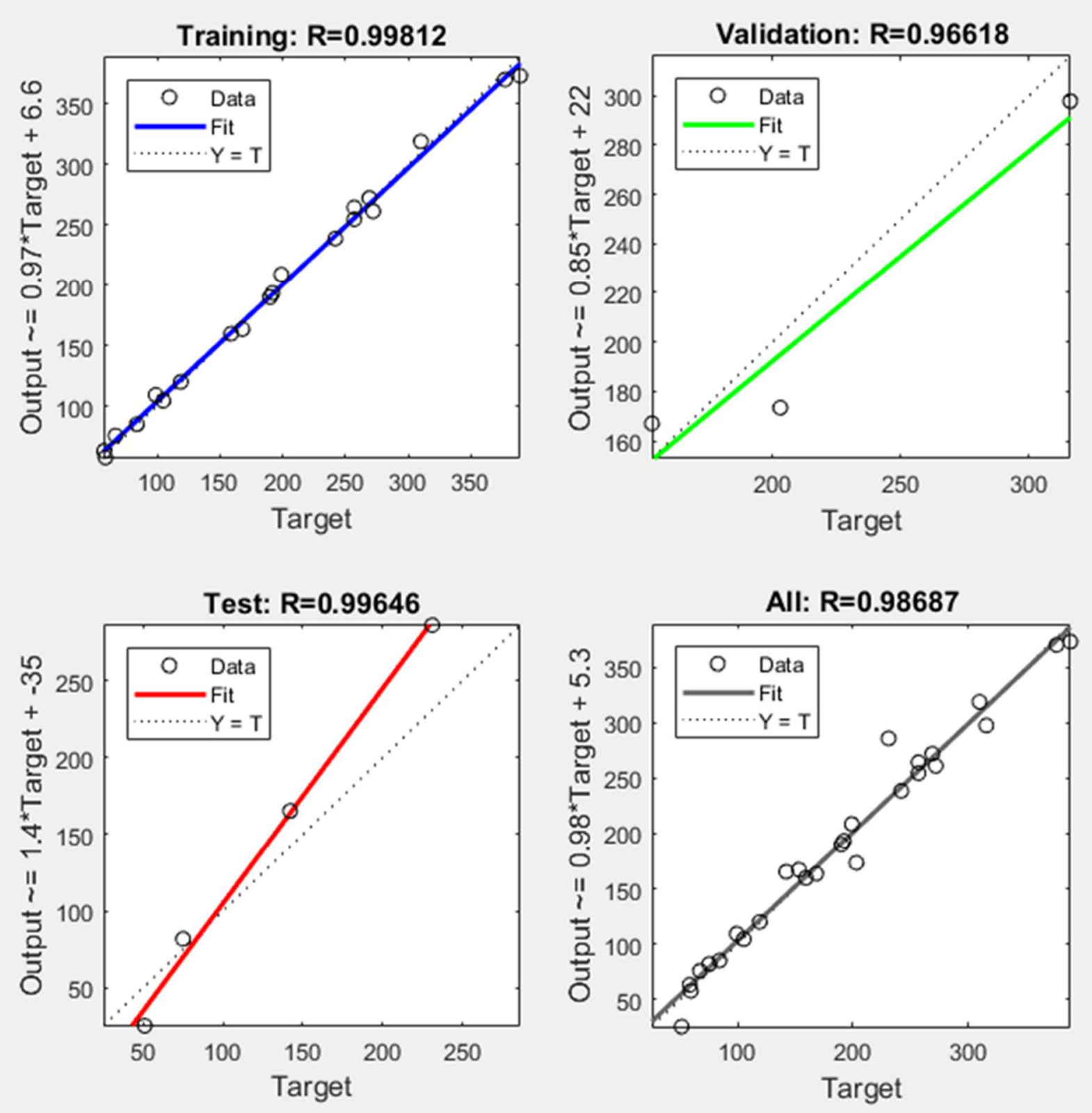

Figure 51: 75\% Training, 10\% Validation, 15\% Testing and 15 Hidden Neurons

75\% Training, 15\% Validation, 10\% Testing and 5 Hidden Neurons 

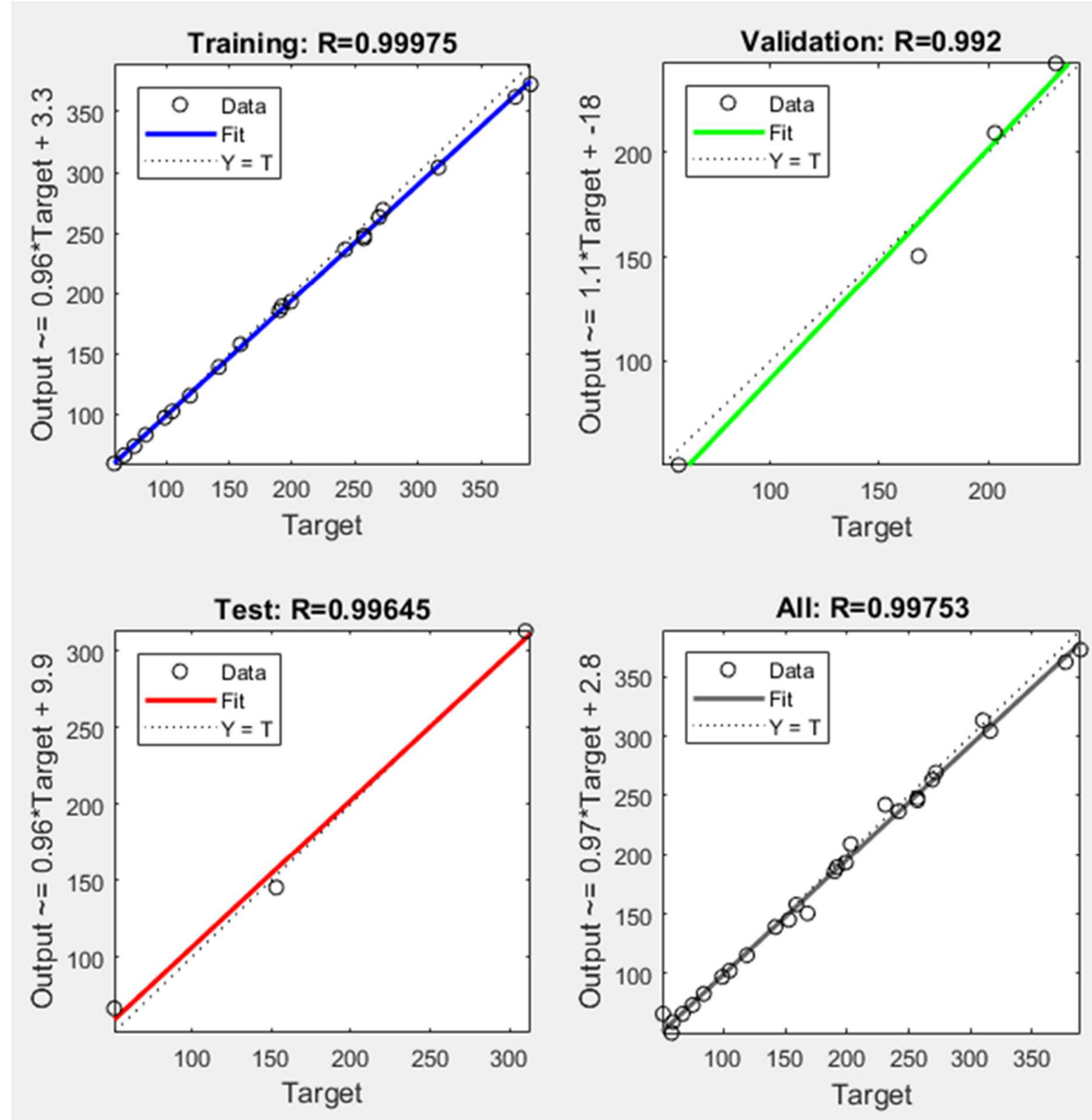

Figure 52: 75\% Training, 15\% Validation, 10\% Testing and 5 Hidden Neurons

75\% Training, 15\% Validation, $10 \%$ Testing and 15 Hidden Neurons 

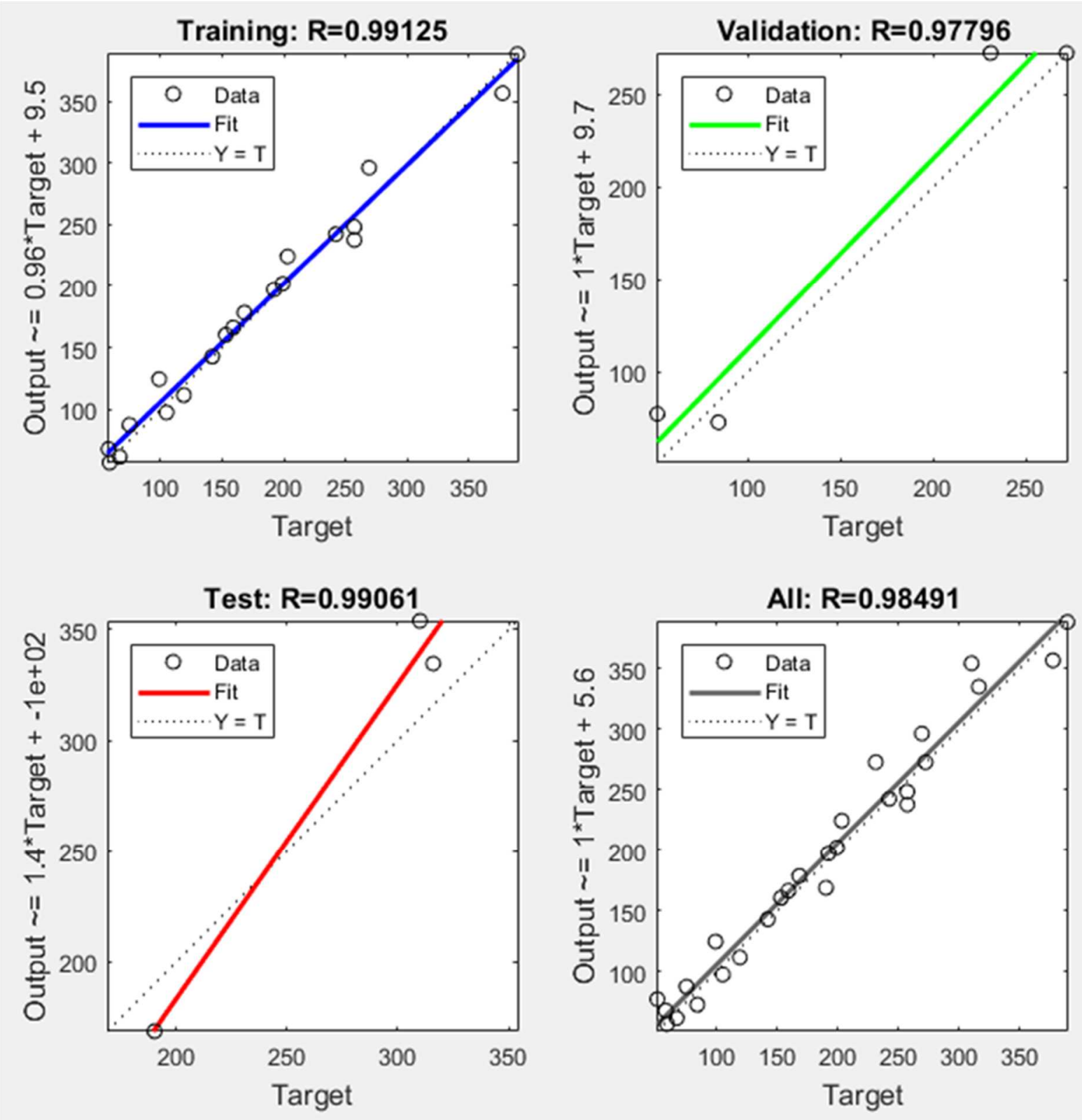

Figure 53: 75\% Training, 15\% Validation, 10\% Testing and 15 Hidden Neurons

70\% Training, 20\% Validation, 10\% Testing and 5 Hidden Neurons 

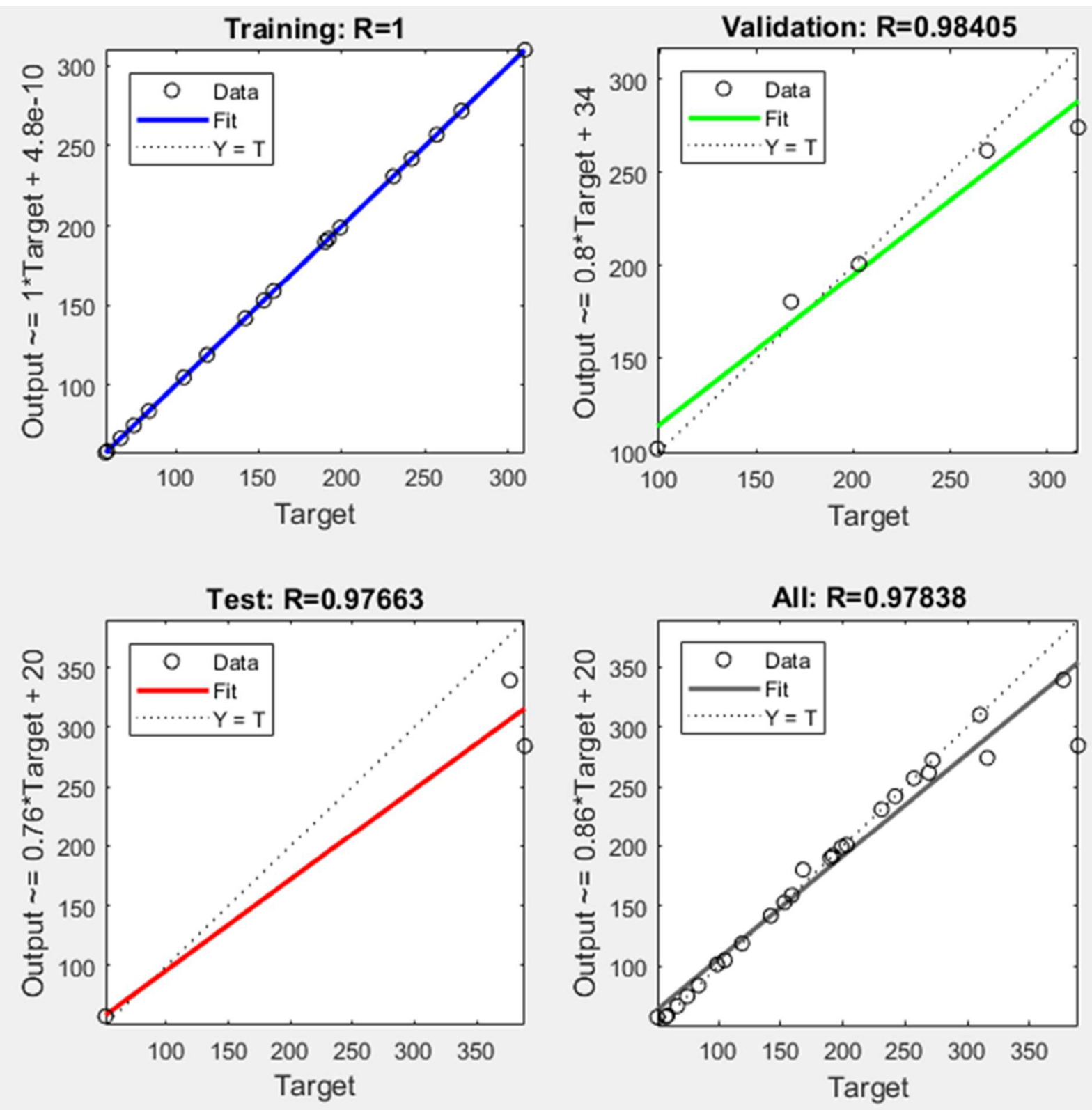

Figure 54: 70\% Training, 20\% Validation, 10\% Testing and 5 Hidden Neurons

70\% Training, 20\% Validation, $10 \%$ Testing and 15 Hidden Neurons 

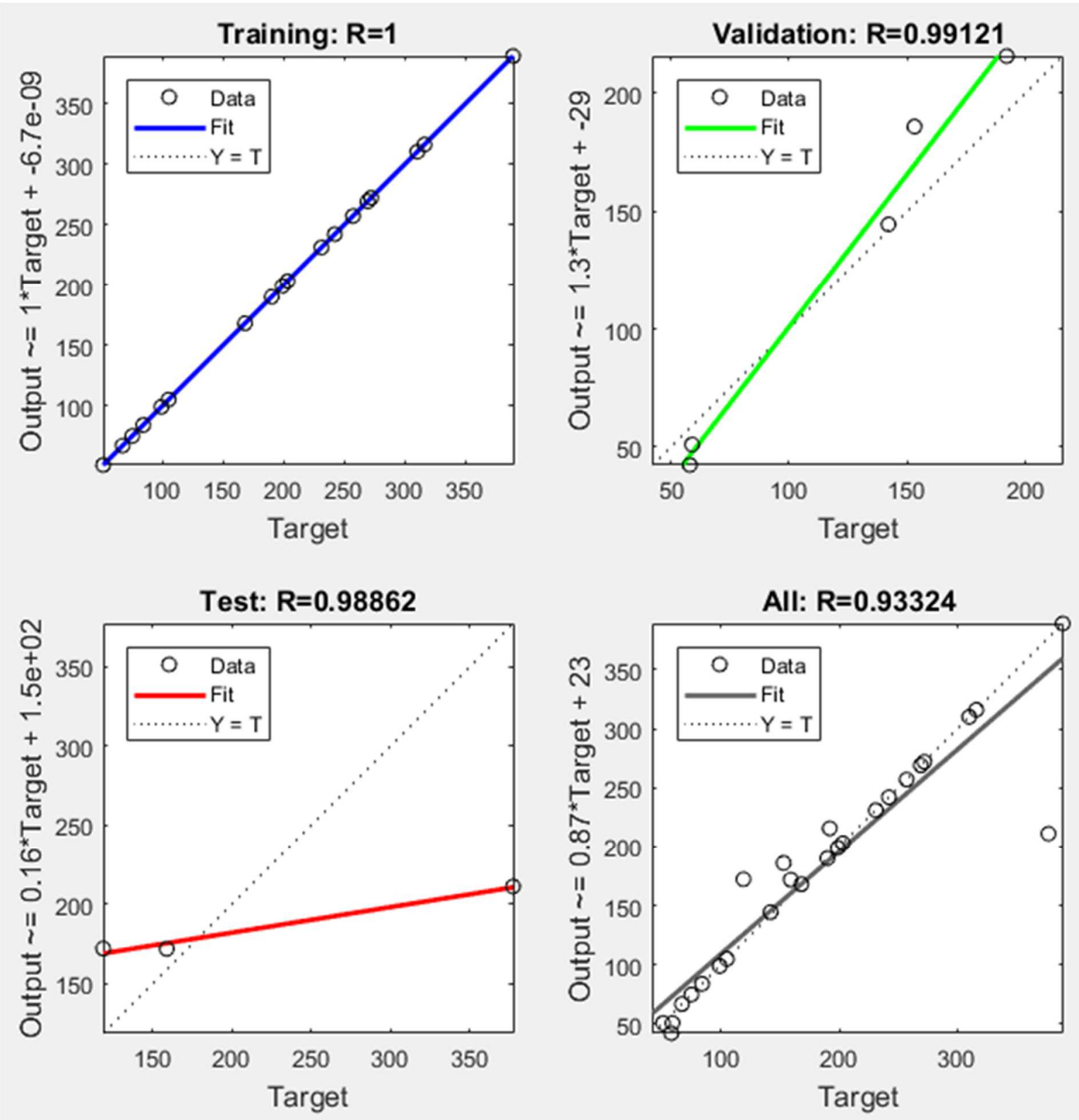

Figure 55: 70\% Training, 20\% Validation, 10\% Testing and 15 Hidden Neurons 


\section{Conclusion}

From this research it can be concluded that the surface flow rate of gas can be accurately determined given the completion design data. The use of Matlab's Neural Network Fitting application shows a regression value near 1.0 for a majority of the runs completed varying the training $\%$, validation $\%$, testing $\%$ and number of hidden neurons. This regression value near 1.0 indicates a strong trend between the completion design (inputs) and the surface gas flow rate (output). The spinner flow scanner was effective in determining the gas flow from different intervals of the horizontal wellbore. The optimal neural network design uses $70 \%, 20 \%$, and $10 \%$ of the data set for training, validation, and testing, receptively.

A shortcoming with this research is the limited amount of data that was available. There was 27 stages total being evaluated with 10 inputs and 1 output for each stage. It would have been beneficial to of been able to obtain more data about the well including the rock properties in the lateral. 


\section{Works Cited}

Continuous and fullbore spinner flowmeters. (2017, March 10). Retrieved from petrowiki: http://petrowiki.org/Continuous_and_fullbore_spinner_flowmeters

Production logging. (2017, March 9). Retrieved from petrowiki: http://petrowiki.org/Production_logging

Baig, A. M., Urbanic, T., Goldstein, S., \& ESG Solutions. (2012). Assesing the Spacing of Stages in Plug and Perf Completions in the Marcellus Shale. Society of Petroleum Engineers.

Cheng, Y. (2010). Impacts of the Number of Perforation Clusters and Cluster Spacing on Production Performance of Horizontal Shale Gas Wells. Society of Petroleum Engineers.

Filchock, J. J., Aminian, K., \& Ameri, S. (2016). Impact of Completion Parameters on Marcellus Shale Production. Society of Petroleum Engineers.

Izadi, G., Junca, J.-P., \& Cade, R. (2014). Production Performance in Marcellus Shale: Multidisciplinary Study of Hydraulic Fracturing. Society of Petroleum Engineers.

McKinley, R. M. (1982). Production Logging. Society of Petroleum Engineers.

Parijat, M. (2013). Principles of Production Logging. Oilfield Review, 63-64.

Schweitzer, R., \& Bilgesu, I. H. (2009). The Role of Economics on Well and Fracture Design Completions of Marcellus Shale Wells. Society of Petroleum Engineers.

Shelley, R., Nejad, A., Guliyev, N., Raleigh, M., \& Matz, D. (2014). Understanding Multi-Fractured Horizontal Macellus Completions. Society of Petroleum Engineers.

Walker, K., Wutherich, J., Terry, J., Schlumberger, Shreves, J., Caplan, J., et al. (2012). Improving Production in the Marcellus Shale Using an Engineered Completion Design: A Case Study. Society of Petroleum Engineers.

Zarmirian, M., Aminian, K., \& Ameri, s. (2016). Measuring Marcellus Shale Petrophysical Properties. Society of Petroleum and Natural Gas Engineers. 